\title{
MEMOIR ON THE GENERAL THEORY OF SURFACES AND RECTILINEAR CONGRUENCES*
}

BY

\author{
GABRIEL M. GREEN
}

\section{CONTENTS}

Introduction $\ldots \ldots \ldots \ldots \ldots \ldots \ldots \ldots \ldots \ldots \ldots \ldots \ldots \ldots \ldots \ldots \ldots \ldots$

1. Fundamental equations for a surface referred to its asymptotic curves . . . . . . 85

2. Reciprocal congruences and the relation $R \ldots \ldots \ldots \ldots \ldots \ldots \ldots \ldots \ldots \ldots \ldots$

3. The developables of the congruences $\Gamma$ and $\Gamma^{\prime} \ldots \ldots \ldots \ldots \ldots \ldots \ldots \ldots \ldots$

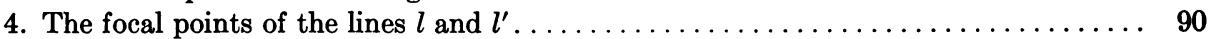

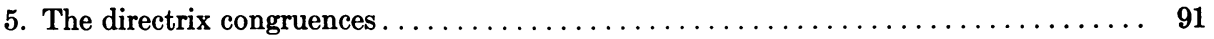

6. Some general properties of reciprocal congruences $\ldots \ldots \ldots \ldots \ldots \ldots \ldots \ldots \ldots \ldots \ldots$

7. The osculating quadric, and its connection - with reciprocal congruences. The canonical quadric $\ldots \ldots \ldots \ldots \ldots \ldots \ldots \ldots \ldots \ldots \ldots \ldots \ldots \ldots \ldots \ldots \ldots$

8. Congruences conjugate and harmonic to a surface . . . . . . . . . . . . 99

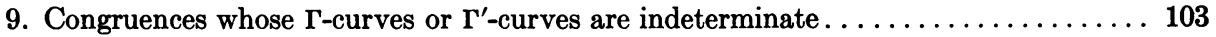

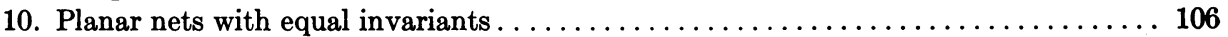

11. The perspectives of the asymptotic curves on the tangent plane, and their four-point

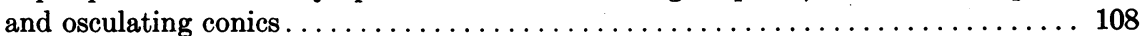

12. The canonical congruences of the first and second kinds. Characterization and

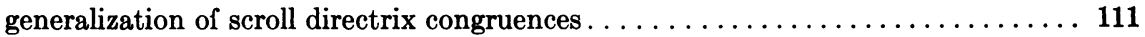

13. Darboux's canonical expansion in non-homogeneous coördinates . . . . . . . . 115

14. The completed characterization of the canonical tetrahedron $\ldots \ldots \ldots \ldots \ldots \ldots \ldots$

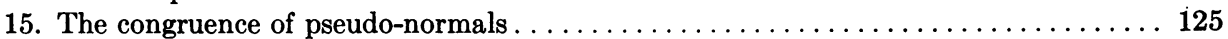

16. General theorems on conjugate nets $\ldots \ldots \ldots \ldots \ldots \ldots \ldots \ldots \ldots \ldots \ldots \ldots \ldots \ldots$

17. Pseudo-geodesics, union curves, and generalized surfaces of Voss . . . . . . . . 136

18. Dual considerations; curves of Darboux and Segre; axis and ray congruences...... 140

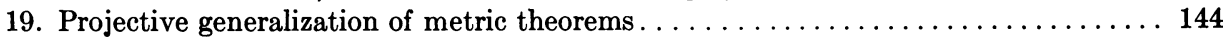

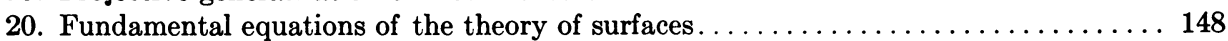

\section{INTRODUCTION}

The metric differential geometry of surfaces undoubtedly owes much of its wide development to the fact that the notion of normal to the surface is a simple one both geometrically and analytically. Indeed, it would hardly be an exaggeration to say that all of the metric theory of surfaces is founded on this notion of normal, if only because the direction cosines of the normal

* Presented to the Society, September 5, 1916 and December 27, 1917.

Editors Note. The manuscript of this memoir was under consideration by the Editorial Committee when the untimely death of Dr. Green cut short a career which was full of promise. The Editors are pleased to publish the memoir in the form given it by the author. 
enter so essentially in the analytic formulation involved in the Gauss differential equations.

Not until recent years had any systematic study of the differential geometry of surfaces from a projective point of view been attempted. Many important discoveries have been made, but one thing has always seemed to be lacking, viz., a satisfactory projective substitute for the normal to the surface. It was chiefly in a search for such a substitute that the investigations of the present paper were carried out; but certain new concepts which were evolved proved to have such a wide field of applicability that the work expanded to very comprehensive proportions. Only some of our results have reached a definitive form, but under existing circumstances it seems to us wiser to present these in detail, and to outline others which at present are little more than suggestive speculations, rather than to wait for the completion of investigations which may have to be discontinued at any time.

The concept which has played the central rôle in our researches is a very general one which we had previously found fundamental in another connection;* in the present memoir the particularized concept may be described as follows: At every regular point of a curved surface there exists an osculating quadric, which is cut by the tangent plane of the surface in the asymptotic directions at the point of contact. A line $l$ lying in the tangent plane of the surface, and a line $l^{\prime}$ passing through the point of contact, $P$, of this tangent plane-but not lying in it-are said to be reciprocal to each other if they are reciprocal polars of the osculating quadric of the surface at the point $P$. This reciprocal relation is the particular case of the relation $R$ which arises when the surface is referred to its asymptotic net.

Let $\Gamma$ be a congruence composed of lines $l$, one line $l$ lying in each of the tangent planes of the non-developable surface $S$. This congruence determines uniquely a reciprocal congruence, $\Gamma^{\prime}$, composed of the lines $l^{\prime}$ reciprocal to the lines $l$, and conversely. Further specialization of the relation between a pair of reciprocal congruences affords a very powerful method of characterizing geometrically particular congruences associated with a given surface. In fact, besides obtaining in this way certain new congruences, among them the projective substitute for the congruence of normals which formed the original object of our researches, we have been able to characterize in terms of the reciprocal relation all of the important projectively defined congruences thus far discovered. This relation therefore unifies a large number of otherwise isolated concepts.

${ }^{*}$ This general concept is one which we have called the relation $R$ in several communications to the Society. It is fully described in a note which appeared in the Proce eding of the National Academy of Sciences, vol. 3 (1917), pp. 587-592, in which were stated a number of the results of the present paper. Cf. also G. M. Green, Some geometric characterizations of isothermal nets on a curved surface, these $\mathrm{Tr}$ a n a c t i o n s, vol. 18 (1917), pp. 480-488. 
The developables of the congruence $\Gamma$ correspond to a net of curves on the surface $S$ which we call the $\Gamma$-curves. Likewise, the developables of the congruence $\Gamma^{\prime}$ cut $S$ in a net of curves which we call the $\Gamma^{\prime}$-curves. A study of these developables and of the focal points of the lines $l$ and $l^{\prime}$ gives rise to a large number of interesting theorems. In particular we may mention here a new characterization of Wilczynski's directrix congruences, as a pair of reciprocal congruences whose $\Gamma$ - and $\Gamma^{\prime}$-curves coincide.

The tangents to the two $\Gamma$-curves which pass through a point of the surface -we call them the $\Gamma$-tangents of the point-give rise by harmonic reflection in the asymptotic tangents to two lines, the reflected $\Gamma$-tangents, each of which meets the line $l$ in the focal point of $l$ which corresponds to it. This proves to be a valuable property, and is in fact the fundamental relation between the $\Gamma$-curves and the focal points of the line $l$. If, further, each $\Gamma$-tangent is the reflection of the other, then the net of $\Gamma$-curves is a conjugate net, and the congruence is said to be harmonic to the surface $S$. In this case the $\Gamma^{\prime}$ curves also form a conjugate net, and the congruence $\Gamma^{\prime}$ is then said to be conjugate to the surface $S$. We are here borrowing the terminology of Guichard, who speaks of congruences conjugate or harmonic to a réseau. Among several geometric characterizations of congruences harmonic and conjugate to a surface is the one obtained from the general property just stated: a congruence $\Gamma$ is harmonic if and only if each of its $\Gamma$-tangents meets the line $l$ in the focal point of $l$ which corresponds to the other $\Gamma$-tangent.

An especially interesting case arises when either of the nets of $\Gamma$ - and $\Gamma^{\prime}$ curves becomes indeterminate, or when both do. If the $\Gamma$-curves are indeterminate, the congruence $\Gamma$ consists of the lines of a plane, but the reciprocal congruence is conjugate to the surface, and need not be degenerate. A dual situation subsists if the $\Gamma^{\prime}$-curves are indeterminate, in which case the congruence $\Gamma$ is harmonic to the surface. These facts afford a method for determining congruences conjugate or harmonic to a surface without any integration whatever, whether the asymptotic curves be known or not. If both the $\Gamma$ and $\Gamma^{\prime}$-curves are indeterminate, they must be the directrix curves of the surface. The case in which the $\Gamma^{\prime}$-curves are indeterminate is very intimately connected with a geometric characterization of planar nets with equal invariants which has recently been published.*

The perspectives of the asymptotic curves on the tangent plane, the center of projection being a point on the line $l^{\prime}$, play an importart part in the definition of several new congruences. If $P$ be the point of the surface through which $l^{\prime}$ passes, then there exists a pencil o conics which have contact of the third order with one of the projected asymptotics at $P$, and another pencil

* G. M. Green, Plane nets with equal invariants, A n n als of $\mathrm{M}$ at he matics, vol. 19 (1918), pp. 246-250. 
of conics which have contact of the third order with the other projected asymptotic at $P$. Among each of these pencils of four-point conics exists an osculating conic. Two lines are said to be reciprocal polars with respect to a conic if either passes through the pole of the other. Then at each point $P$ of the surface $S$ there exists just one line $l^{\prime}$ such that with any point on $l^{\prime}$ as a center of projection the asymptotic tangents at $P$ are reciprocal polars with respect to each of the conics having four-point contact with the projected asymptotics at $P .{ }^{*}$ We call this line $l^{\prime}$ the canonical edge of the second kind, and the congruence of lines $l^{\prime}$ thus defined the canonical congruence of the second kind. The reciprocal line $l$ and congruence $\Gamma$ we call the canonical edge and canonical congruence of the first kind. A line $l$ of the latter congruence cuts the corresponding asymptotic tangents in two points, the first and second canonical vertices.

Another pair of reciprocal congruences which may be defined in terms of the concepts thus introduced is one which becomes Sullivan's pair of scroll directrix congruences if the surface be ruled.

The canonical edges of the first and second kinds meet the canonical quadric in four points which we choose as the vertices of a local tetrahedron of reference. If, further, the unit point of the coördinate system be properly chosen, the equation of the surface in non-homogeneous coördinates may be obtained in the form

$$
z=x y+\frac{1}{6}\left(x^{3}+y^{3}\right)+\frac{1}{2}\left(I^{\prime} x^{3} y+J^{\prime} x y^{3}\right)+\cdots,
$$

where $I^{\prime}, J^{\prime}$, and all succeeding coefficients are projective differential invariants of the surface. This, together with a similar expansion of the form

$$
z=x y+\frac{1}{6}\left(x^{3}+y^{3}\right)+\frac{1}{24}\left(I x^{4}+J y^{4}\right)+\cdots
$$

was discovered by Darboux, $\uparrow$ who gave no characterization of the tetrahedron for either expansion. The second of the above developments was first characterized by Wilczynskif; his description of the unit point of the coördinate system is not, however, purely geometric. We supply a description which is entirely free from any analytic formulation, and which is applicable to the coördinate systems giving rise to both of Darboux's developments.

${ }^{*}$ The four-point and osculating conics of the projected asymptotics coincide, of course, with the four-point and osculating conics of the asymptotics themselves. We prefer to state the theorems in terms of the projected asymptotics, however, to obviate the necessity of continually recalling that the four-point and osculating conics of the asymptotics lie in the tangent plane of the surface.

†Bulletin des sciences mathématiques et astronomiques, ser. 2 , vol. 4 (1880), pp. 348-384.

The invariants in the two expansions are connected by the equations $I^{\prime}=2 J, J^{\prime}=2 I$.

$\ddagger$ Projective differential geometry of curved surfaces, second memoir, these $\mathrm{Tr}$ ans a cti o n s, vol. 9 (1908), pp. 79-120, $\$ \$ 6,7$. Cf. also the fifth memoir, these Trans a c t i o n s, vol. 10 (1909), p. 289. 
Perhaps more important than any of the congruences already described is what we call the pseudo-normal congruence, which ought in the projective theory to play a rôle analogous to that of the congruence of normals in the metric theory. The developables of the congruence of normals meet the surface in a conjugate net, so that a projective substitute for such a congruence ought to be a uniquely determined congruence which is likewise conjugate to the surface. We define a congruence having the desired qualifications as follows: The directrix of the first kind and the canonical edge of the first kind corresponding to a point $P$ of the surface meet in a point $P^{\prime}$. The pseudonormal of the point $P$ is the reciprocal of the line which is the harmonic conjugate of the directrix of the first kind with respect to the canonical edge of the first kind and the line $P P^{\prime}$.

Having by this means provided a projectively defined substitute for the normals to the surface, we may generalize a large number of important theorems of metric differential geometry. Suggestions are made towards the end of the paper for the projective generalization of such configurations as geodesics, surfaces of Voss, triply orthogonal systems, families of Lamé, surfaces and congruences of Guichard, surfaces of Weingarten, and isothermic surfaces. The only ones of these generalizations which are discussed in some detail are those of geodesics and surfaces of Voss. With Miss Pauline Sperry* we define a union curve of a congruence $\Gamma^{\prime}$ as a curve on the surface, whose osculating plane at each point contains the line $l^{\prime}$ of $\Gamma^{\prime}$ passing through that point. There exist a two-parameter family of union curves, and if the congruence $\Gamma^{\prime}$ is the congruence of pseudo-normals we call the union curves pseudo-geodesics. A generalized surface of Voss, then, is defined as a surface on which there exists a conjugate net formed entirely of pseudo-geodesics. These surfaces are the analogue of surfaces of Voss, which are characterized by possessing a conjugate net of geodesics. This conjugate net has equal tangential invariants, and so also does the conjugate net of pseudo-geodesics on a generalized surface of Voss. This property furnishes strong evidence of the desirability of replacing the normal congruence by a congruence $\Gamma^{\prime}$ which is not merely uniquely determined, and projectively, by the surface, but is in addition conjugate to the surface. For, we prove the theorem that if the union curves of a congruence $\Gamma^{\prime}$ contain among them two one-parameter families which form a conjugate net, then a necessary and sufficient condition that this conjugate net have equal tangential invariants is that the congruence $\Gamma^{\prime}$ be conjugate to the surface.

A space dualization of the definition of union curves leads to the definition of what we call the adjoint union curves of a congruence $\Gamma$. Corresponding

\footnotetext{
* Properties of a certain projectively defined two-parameter family of curves on a general surface, American Journal of $\mathrm{M}$ athematics, vol. 40 (1918), pp. 213-224.
} 
by duality to the osculating plane of a curve $C$, we have the point of the edge of regression of the developable formed by the tangents conjugate to the tangents of $C$. This point is called the first Laplace transform of the corresponding point of the curve $C$. A curve is called an adjoint union curve of a congruence $\Gamma$ if the first Laplace transforms of the points of the curve lie on the corresponding lines $l$ of $\Gamma$.

The adjoint union curves of a congruence $\Gamma$ coincide with the union curves of the reciprocal congruence $\Gamma^{\prime}$ if and only if the surface is a quadric; but on any non-ruled surface whatever there are three one-parameter families of curves which are at the same time adjoint union curves for certain congruences $\Gamma$ and union curves for the reciprocal congruences $\Gamma^{\prime}$. We call these curves the curves of Segre, because their tangents were defined by Segre, ${ }^{*}$ though in an entirely different way. The three one-parameter families conjugate to the three families of curves of Segre are Darboux's curves of quadric osculation, $\dagger$ which we call curves of Darboux. The latter curves are therefore characterized geometrically in terms of our characterization of the curves of Segre.

There is, however, a close connection between the union curves and adjoint union curves, in certain cases, which may be described as follows. In another memoir, $\ddagger$ we called associate conjugate nets two conjugate nets, such that at each point the two tangents to the curves of either net separate harmonically the tangents to the curves of the other net. The connection referred to is involved in the theorem, that if there exists a conjugate net formed of union curves of a congruence $\Gamma^{\prime}$, then the associate conjugate net consists of adjoint union curves of the reciprocal congruence $\Gamma$, and conversely. This theorem throws light on several results concerning conjugate nets and the general theory of congruences to be found in the paper last cited. Moreover, the results obtained in the present memoir justify the expectation that a large part of the theory of rectilinear congruences, with related transformation theory of surfaces, may be based on the methods here used.

The memoir closes with a discussion, rather in the nature of suggestions for metric concepts mentioned above, and of a certain system of differential equations, a generalization of the Gauss equations, which may serve as a basis for a projective theory of surfaces.

* C. Segre, Complementi alla teoria delle tangenti coniugate di una superficie. $\mathrm{R}$ e $\mathrm{n} \mathrm{d} \mathrm{i}-$ c onti della R. Ac cademia dei Lincei, ser. 5, vol. 17 2 (1908), pp. 405-412.

† G. Darboux, Sur le contact des courbes et des surfaces. B ulletin des Sciences M a t h e m a t i ques, ser. 2, vol. 4 (1880), pp. 348-384, in particular pp. 356 et seq.

$\ddagger$ G. M. Green, Projective differential geometry of one-parameter families of space curves, and conjugate nets on a curved surface, second memoir, A m e rican Journal of Mathem a t i c s, vol. 38 (1916), pp. 287-324. Cf. p. 314. 


\section{Fundamental equations for a suRface ReFERRed to its asymptotic CURVES}

Let $y^{(1)}, y^{(2)}, y^{(3)}, y^{(4)}$ be the homogeneous coördinates of a point $y$ in a projective space of three dimensions. We shall consider a surface $S$ defined by the equations

$$
y^{(k)}=y^{(k)}(u, v) \quad(k=1,2,3,4),
$$

where the functions $y^{(k)}$ possess all continuous derivatives which we may need in our subsequent discussion. We shall suppose the surface to be nondevelopable, ${ }^{*}$ and referred to its asymptotic net. Then the four functions $y^{(k)}$ are a fundamental system of solutions of a completely integrable system of partial differential equations

$$
\begin{aligned}
y_{u u}+2 a y_{u}+2 b y_{v}+c y & =0, \\
y_{v v}+2 a^{\prime} y_{u}+2 b^{\prime} y_{v}+c^{\prime} y & =0 .
\end{aligned}
$$

By a transformation

$$
y=\lambda(u, v) \bar{y},
$$

where

$$
\lambda_{u}=-a \lambda, \quad \lambda_{v}=-b^{\prime} \lambda,
$$

system (2) may be transformed into a system of the same form for which $a$ and $b^{\prime}$ are both zero. $\dagger$ This system is

$$
\begin{aligned}
y_{u u}+2 b y_{v}+f y & =0, \\
y_{v v}+2 a^{\prime} y_{u}+g y & =0,
\end{aligned}
$$

\footnotetext{
* In most writings on differential geometry, attention is confined exclusively to "regular" -as distinguished from "exceptional"-points of a configuration. When such is the case, a writer usually refrains from pointing out that exceptions to his theorems may exist, or at most makes a concession by a liberal use of the words "in general." From the point of view of rigor, then, his theorems and proofs are incomplete, if not entirely wrong. To avoid any possible misunderstanding, we shall state here explicitly certain assumptions which we feel are usually tacitly made by differential geometers, and which we shall make throughout the present paper. An example will explain the matter sufficiently well. A surface is developable if, in the language of metric geometry, the total curvature $K$ is identically zero. Now, the failure of a theorem will usually arise from the vanishing of $K$, rather than from the fact that the surface is developable. In other words, the vanishing of $K$ at a single point may invalidate the statement of the theorem for any region containing that point. For our purposes, however, it is far more convenient to state that the theorem fails if the surface is developable, including in this statement, by implication, all cases where $K=0$. A proposition, then, is true except at those points where it fails, and we specify these points not by telling what equation subsists at these points, but by describing what would happen if this equation were true at all points. When, therefore, we say "except if the surface is developable," we imply the broader statement "except at those points for which $K=0$."

$\dagger$ E. J. Wilczynski, Projective differential geometry of curved surfaces, first memoir, these Trans a ction s, vol. 8 (1907), pp. 233-260. Cf. p. 246.
} 
where

$$
f=c-a_{u}-a^{2}-2 b b^{\prime}, \quad g=c^{\prime}-b_{v}^{\prime}-b^{\prime 2}-2 a a^{\prime} .
$$

The coefficients of equations (4) are connected by the conditions of complete integrability

$$
\begin{aligned}
a_{u u}^{\prime}+g_{u}+2 b a_{v}^{\prime}+4 a^{\prime} b_{v} & =0, \\
b_{v v}+f_{v}+2 a^{\prime} b_{u}+4 b a_{u}^{\prime} & =0, \\
g_{u u}-f_{v v}-4 g a_{u}^{\prime}-2 a^{\prime} f_{u}+4 f b_{v}+2 b g_{v} & =0 .
\end{aligned}
$$

We shall deal with the system in its canonical form (4) exclusively; our analytic results will, however, be expressible entirely in terms of the coefficients and variables of equations (2), in virtue of equations (3) and (4a).

\section{Reciprocal Congruences and the Relation $R$}

We shall denote by $C_{u}$ a curve $v=$ const., and by $C_{v}$ a curve $u=$ const., on the surface $S$. Since the parametric net is not conjugate, the ruled surface formed by the tangents to the curves $C_{u}$ at the points of a fixed curve $C_{v}$ is not developable. Let us denote such a ruled surface by $R^{(\boldsymbol{*})}$, and by $R^{(v)}$ the similar parametric ruled surfaces formed by tangents to the curves $C_{v}$.

Through a point $y$ of the surface pass two parametric tangents. The points

$$
\rho=y_{u}-\beta y, \quad \sigma=y_{v}-\alpha y
$$

lie on these tangents, and the line $\rho \sigma$ is a line $l$ in the tangent plane to the surface $S$ at $y$, and does not pass through $y$. If $\alpha$ and $\beta$ are functions of $(u, v)$, equations (6) define a line $l$ for each tangent plane of the surface, and by suitably choosing these functions $\alpha$ and $\beta$ any arbitrary congruence $\Gamma$ may be defined which is formed of lines $l$, one and only one corresponding to and lying in each tangent plane of the surface, and no one of which passes through the corresponding point of the surface.

Corresponding to a line $l$ in the tangent plane at $y$, we may define geometrically another line $l^{\prime}$ which passes through the point $y$ but does not lie in the tangent plane, as follows. The parametric ruled surface $R^{(u)}$ has for its tangent plane at the point $\rho$ a plane which passes through the generator $y \rho$ of the ruled surface, and which does not coincide with the tangent plane to $S$ at $y$ since $R^{(u)}$ is not a developable. Similarly, the tangent plane to the ruled surface $R^{(v)}$ at the point $\sigma$ passes through the parametric tangent $y \sigma$ and does not coincide with the tangent plane to $S$ at $y$. The two tangent planes to $R^{(u)}$ and $R^{(v)}$ at $\rho$ and $\sigma$ respectively therefore intersect in a definite line $l^{\prime}$ which passes through the point $y$, and it remains to determine another point on this line. There must be a point on $l^{\prime}$ given by an expression of the form

$$
z=y_{u v}+\lambda y_{u}+\mu y_{v}
$$


The plane of the points $y, z, \rho$ is to be tangent to the ruled surface $R^{(u)}$ at $\rho$, and therefore must contain in it the tangent to the curve traced by $\rho$ as the point $y$ moves along a curve $C_{v}$. A point on this tangent is given by

$$
\rho_{v}=y_{u v}-\beta y_{v}-\beta_{v} y
$$

this is to lie in the plane $y z \rho$, which can occur if and only if $\mu=-\beta$. Similarly, the plane $y z \sigma$ is tangent to the ruled surface $R^{(v)}$ at $\sigma$ if and only if $\lambda=-\alpha$. The line $l^{\prime}$ is therefore the line joining the point $y$ to the point

$$
z=y_{u v}-\alpha y_{u}-\beta y_{v} .
$$

If, conversely, any line $l^{\prime}$ passing through the point $y$, but not lying in the tangent plane of $y$, be given, a corresponding line $l$ in the tangent plane but not passing through $y$ is uniquely determined. For, any line $l^{\prime}$ may be defined as a line $y z$, where $z$ is an expression of the form (7). The plane of the points $y, y_{u}, z$ passes through the generator $y y_{u}$ of the skew ruled surface $R^{(u)}$, and is therefore tangent to $R^{(u)}$ in one and only one point, viz., the point $\rho=y_{u}-\beta y$. The point $\sigma=y_{v}-\alpha y$ is determined in a similar way, as the point in which the plane of the points $y, y_{v}, z$ touches the ruled surface $R^{(v)}$. The line $l$ which joins these points $\rho$ and $\sigma$ is therefore uniquely defined in terms of the line $l^{\prime}$.

We shall call either of the lines $l, l^{\prime}$ which are related to each other in the manner just described, the reciprocal of the other, and shall also speak of the congruence $\Gamma$ of lines $l$ and the congruence $\Gamma^{\prime}$ of lines $l^{\prime}$ as reciprocal congruences, and of either as the reciprocal of the other. The geometric relation between two reciprocal congruences may for want of a better name be called the relation $R^{*}$.

It is easily verified that if the differential equations be in the form (2) instead of the canonical form (4), the line $l$ joining the points.

$$
\rho=y_{u}-\beta y, \quad \sigma=y_{v}-\alpha y
$$

is reciprocal to the line $l^{\prime}$ joining the points $y$ and

$$
z=y_{u v}-\alpha y_{u}-\beta y_{v} .
$$

* This relation may of course be defined in exactly the same way whatever the parametric net may be, provided it is not conjugate. We have, in fact, established the fundamental formulas for the most general case, in connection with a metric problem, starting with a generalization of the Gauss differential equations. Cf. G. M. Green, Some geometric characterizations of isothermal nets on a curved surface, these $\mathrm{T}$ ra $\mathrm{n}$ a c t i o n s, vol. 18 (1917), pp. 480-488. In this paper we referred to two lines in the relation $R$ as conjugate to each other. A better justification for our use of the name reciprocal in the case of the present paper, where the parametric net is asymptotic, will be given later.

We hope to be able soon to publish our results concerning the relation $R$ in purely projective form. Cf., however, a note in the Proceedings of the National Academ of S cien ces, vol. 3 (1917), pp. 587-592. 
The configuration formed by the asymptotic net and a pair of congruences in the relation $R$ with respect to that net is a projectively self-dual one, in the sense that by a polar reciprocation the configuration goes over into one of the same kind. In fact, each point of $S$ and the corresponding tangent plane are transformed into a tangent plane of a surface $S_{1}$ and its point of contact. The points of an asymptotic on $S$ correspond to the osculating planes of an asymptotic on $S_{1}$, i. e., to the tangent planes of $S_{1}$ along an asymptotic. We may say then that the asymptotics of $S$ correspond to the asymptotics of $S_{1}$. A line $l$ in a tangent plane of $S$ transforms into a line $l_{1}^{\prime}$ through the corresponding point of $S_{1}$, and a line $l^{\prime}$ through a point of $S$ transforms into a line $l_{1}$ in the corresponding tangent plane of $S_{1}$. If $l$ and $l^{\prime}$ are reciprocals of each other, with respect to the surface $S$, then their respective transforms $l_{1}^{\prime}$ and $l_{1}$ are reciprocals of each other with respect to the surface $S_{1}$. For, a ruled surface $R^{(u)}$ of asymptotic tangents of $S$ corresponds to a ruled surface $R_{1}^{(u)}$ of asymptotic tangents of $S_{1}$, and the tangent plane to $R^{(u)}$, with its point of contact $\rho$-which tangent plane contains the line $l^{\prime}$-goes over into the point $\rho_{1}$ with the corresponding tangent plane to $R^{(u)}$-which tangent plane contains the line $l_{1}^{\prime}$.

\section{The Developables of the congruences $\Gamma$ and $\Gamma^{\prime}$}

To every curve on the surface $S$ corresponds a ruled surface of the congruence $\Gamma$. We seek the net of curves on $S$ which correspond to the two one-parameter families of developables of $\Gamma$. If the line $\rho \sigma$ is to generate a developable, the points $\rho$ and $\sigma$ must generate two curves, corresponding tangents of which lie in the same plane. A necessary and sufficient condition, then, for a displacement along a developable of $\Gamma$, is that the points $\rho, \sigma$, $d \rho, d \sigma$ lie in a plane. We have, in virtue of equations (4),

$$
\begin{aligned}
& \rho_{u}=y_{u u}-\beta y_{u}-\beta_{u} y=-\beta y_{u}-2 b y_{v}-\left(f+\beta_{u}\right) y, \\
& \rho_{v}=y_{u v}-\beta y_{v}-\beta_{v} y, \\
& \sigma_{u}=y_{u v}-\alpha y_{u}-\alpha_{u} y, \\
& \sigma_{v}=-2 a^{\prime} y_{u}-\alpha y_{v}-\left(g+\alpha_{v}\right) y .
\end{aligned}
$$

Therefore, since $d \rho=\rho_{u} d u+\rho_{v} d v$, etc.,

$$
\begin{aligned}
& d \rho=y_{u v} d v-\beta y_{u} d u-(2 b d u+\beta d v) y_{v}-\left[\left(f+\beta_{u}\right) d u+\beta_{v} d v\right] y, \\
& d \sigma=y_{u v} d u-\left(2 a^{\prime} d v+\alpha d u\right) y_{u}-\alpha y_{v} d v-\left[\left(g+\alpha_{v}\right) d v+\alpha_{u} d u\right] y .
\end{aligned}
$$

The points $\rho, \sigma, d \rho, d \sigma$ lie in a plane if and only if in their expressions the 
determinant of the coefficients of $y_{u v}, y_{u}, y_{v}, y$ is zero, i. e.,

$$
\left|\begin{array}{cccc}
0 & 1 & 0 & -\beta \\
0 & 0 & 1 & -\alpha \\
d v & -\beta d u & -2 b d u+\beta d v & -\left(f+\beta_{u}\right) d u+\beta_{v} d v \\
d u & -2 a^{\prime} d v+\alpha d u & -\alpha d v & -\left(g+\alpha_{v}\right) d v+\alpha_{u} d u
\end{array}\right|=0 .
$$

On expanding this determinant, we find that the differential equation of the developables of the congruence $\Gamma$ is

$$
\begin{aligned}
\left(f+\beta^{2}+\beta_{u}+2 b \alpha\right) d u^{2}+\left(\beta_{v}-\alpha_{u}\right) d u d v & \\
& -\left(g+\alpha^{2}+\alpha_{v}+2 a^{\prime} \beta\right) d v^{2}=0 .
\end{aligned}
$$

The developables of the congruence $\Gamma^{\prime}$ cut the surface $S$ in a net of curves whose differential equation may be found in a similar way. If the line $y z$ is to generate a developable, the points $y, z, d y, d z$ must lie in a plane. We have from (7)

$$
z_{u}=y_{u u v}-\alpha y_{u u}-\beta y_{u v}-\alpha_{u} y_{u}-\beta_{u} y_{v},
$$

where $y_{u u v}$ may be calculated from the first of equations (4):

so that

$$
y_{u u v}=4 a^{\prime} b y_{u}-\left(f+2 b_{v}\right) y_{v}+\left(2 b g-f_{v}\right) y,
$$

$$
\begin{gathered}
z_{u}=-\beta y_{u v}+\left(4 a^{\prime} b-\alpha_{u}\right) y_{u}-\left(f+2 b_{v}-2 b \alpha+\beta_{u}\right) y_{v} \\
+\left(2 b g-f_{v}+f \alpha\right) y, \\
z_{v}=-\alpha y_{u v}-\left(g+2 a_{u}^{\prime}-2 a^{\prime} \beta+\alpha_{v}\right) y_{u}+\left(4 a^{\prime} b-\beta_{v}\right) y_{v} \\
+\left(2 a^{\prime} f-g_{u}+g \beta\right) y,
\end{gathered}
$$

the expression for $z_{v}$ being obtained from that for $z_{u}$ by proper interchanges of letters. We may now write down the value of $d z=z_{u} d u+z_{v} d v$ as a linear expression in $y_{u v}, y_{u}, y_{v}, y$, say

$$
d z=A y_{u v}+B y_{u}+C y_{v}+D y,
$$

where $A, B, C$, and $D$ are linear in $d u$ and $d v$. A necessary and sufficient condition that the points $y, z, d y, d z$ lie in a plane is that in the expressions for $z, d y$, and $d z$ the determinant of the coefficients of $y_{u v}, y_{u}, y_{v}$ be zero, i. e., that

$$
\left|\begin{array}{ccc}
1 & -\alpha & -\beta \\
0 & d u & d v \\
A & B & C
\end{array}\right|=0 .
$$

On substituting the values of $A, B$, and $C$, which may easily be read off from equations (10), and then expanding the determinant, we find that the 
differential equation of the developables of the congruence $\Gamma^{\prime}$ is

$$
\begin{aligned}
\left(f+\beta^{2}+\beta_{u}-2 b \alpha+2 b_{v}\right) d u^{2} & +\left(\beta_{v}-\alpha_{u}\right) d u d v \\
& -\left(g+\alpha^{2}+\alpha_{v}-2 a^{\prime} \beta+2 a_{u}^{\prime}\right) d v^{2}=0 .
\end{aligned}
$$

We shall call the curves corresponding to the developables of the congruence $\Gamma$ the $\Gamma$-curves of the surface, and those corresponding to the developables of the congruence $\Gamma^{\prime}$ the $\Gamma^{\prime}$-curves of the surface.

\section{ThE FOCAL POINTS OF THE LINES $l$ AND $l^{\prime}$}

Each line $l$ of the congruence $\Gamma$ belongs to two developable surfaces of the congruence, and the two points in which $l$ touches the edges of regression of these developables-i. e., the foci or focal points of the line $l$-will now be determined. Any point* on the line $l$ may be defined by an expression of the form

$$
\Phi=\rho+\lambda \sigma .
$$

If $\Phi$ is to be a focal point of the line $l$, then the tangent plane to the surface formed by all the points $\Phi$ must contain in it the line $l$. In other words, the points $\Phi_{u}, \Phi_{v}, \rho, \sigma$ must lie in a plane. We have, using equations (8),

$$
\begin{aligned}
\Phi_{u} & =\rho_{u}+\lambda \sigma_{u}+\lambda_{u} \sigma \\
& =\lambda y_{u v}-(\beta+\alpha \lambda) y_{u}-2 b y_{v}-\left(f+\beta_{u}+\lambda \alpha_{u}\right) y+\lambda_{u} \sigma, \\
\Phi_{v} & =\rho_{v}+\lambda \sigma_{v}+\lambda_{v} \sigma \\
& =y_{u v}-2 a^{\prime} \lambda y_{u}-(\beta+\alpha \lambda) y_{v}-\left[\beta_{v}+\left(g+\alpha_{v}\right) \lambda\right] y+\lambda_{v} \sigma .
\end{aligned}
$$

If the points $\Phi_{u}, \Phi_{v}, \rho, \sigma$ lie in a plane, then the points $\Phi_{u}-\lambda_{u} \sigma, \Phi_{v}-\lambda_{v} \sigma$, $\rho, \sigma$ will also; a necessary and sufficient condition for this is that in the expressions for the latter four quantities the determinant of the coefficients of $y_{u v}, y_{u}, y_{v}, y$ vanish, i. e., that

$$
\left|\begin{array}{cccc}
\lambda & -(\beta+\alpha \lambda) & -2 b & -\left(f+\beta_{u}+\lambda \alpha_{u}\right) \\
1 & -2 a^{\prime} \lambda & -(\beta+\alpha \lambda) & -\left[\beta_{v}+\left(g+\alpha_{v}\right) \lambda\right] \\
0 & 1 & 0 & -\beta \\
0 & 0 & 1 & -\alpha
\end{array}\right|=0 .
$$

This is a quadratic in $\lambda$, the two roots of which, when set in equation (12), will give two values for $\Phi$ which define the two focal points of the line $l$. The focal points of the line $l$ are defined by the expressions

$$
\Phi_{1}=\rho+\lambda_{1} \sigma, \quad \Phi_{2}=\rho+\lambda_{2} \sigma,
$$

* Except the point $\sigma$. The simplifications which generally arise when a single parameter $\lambda$, instead of a homogeneous parameter $\lambda: \mu$, is used, make it worth while to restrict the generality of the argument at first, especially since it is always easy to make the necessary changes in the final formulas to include the most general case. 
where $\lambda_{1}$ and $\lambda_{2}$ are the two roots of the quadratic

$$
\left(g+\alpha^{2}+\alpha_{v}+2 a^{\prime} \beta\right) \lambda^{2}+\left(\beta_{v}-\alpha_{u}\right) \lambda-\left(f+\beta^{2}+\beta_{u}+2 b \alpha\right)=0 .
$$

The focal points of the line $l^{\prime}$ are determined in a similar way. Any point of the line $l^{\prime}$ is defined by an expression of the form

$$
\tau=z+\mu y \text {. }
$$

If the point $\tau$ is to be a focal point of $l^{\prime}$, the points $\tau_{u}, \tau_{v}, \tau, y$ must lie in a plane. A necessary and sufficient condition for this is that in the expressions for $\tau_{u}, \tau_{v}$, and $\tau$, the determinant of the coefficients of $y_{u v}, y_{u}, y_{v}, y$ be zero. Using equations (10) in the calculation of $\tau_{u}$ and $\tau_{v}$, and expanding the determinant just referred to, we find that the focal points of the line $l^{\prime}$ are defined by the expressions

$$
\tau_{1}=z+\mu_{1} y, \quad \tau_{2}=z+\mu_{2} y,
$$

where $\mu_{1}$ and $\mu_{2}$ are the two roots of the quadratic

$$
\begin{aligned}
& \mu^{2}+\left(8 a^{\prime} b-2 \alpha \beta-\alpha_{u}-\beta_{v}\right) \mu \\
& -\left(f+\beta^{2}+\beta_{u}-2 b \alpha+2 b_{v}\right)\left(g+\alpha^{2}+\alpha_{v}-2 a^{\prime} \beta+2 a_{u}^{\prime}\right) \\
& \quad+\left(4 a^{\prime} b-\alpha \beta-\alpha_{u}\right)\left(4 a^{\prime} b-\alpha \beta-\beta_{v}\right)=0 .
\end{aligned}
$$

The actual expressions for $\tau_{1}$ and $\tau_{2}$ are therefore rather complicated; an important point on the line $l^{\prime}$, and one which is, moreover, defined by a rational and much simpler expression, is the harmonic conjugate of the point $y$ with respect to $\tau_{1}$ and $\tau_{2}$, i. e., the point

$$
\tau=z+\frac{1}{2}\left(\mu_{1}+\mu_{2}\right) y=z+\frac{1}{2}\left(2 \alpha \beta-8 a^{\prime} b+\alpha_{u}+\beta_{v}\right) y .
$$

The totality of points $\tau$ constitute a surface which, using a designation due to Koenigs, we shall call the point-conjugate of $S$ with respect to $\Gamma^{\prime}$. As is well known, the point-conjugate of a surface is of the greatest importance in the transformation-theory of surfaces.

\section{The DIRECTRIX CONGRUENCES}

An important pair of reciprocal congruences is the directrix congruences of the first and second kinds, defined by Wilczynski.* The two linear complexes which osculate at a point $y$ the two asymptotics passing through $y$ have in common a linear congruence with directrices $d$ and $d^{\prime}$. The directrix of the first kind, $d$, lies in the tangent plane of the surface, and connects the two points

$$
r=y_{u}-\frac{a_{u}^{\prime}}{2 a^{\prime}} y, \quad s=y_{v}-\frac{b_{v}}{2 b} y .
$$

\footnotetext{
*Curved surfaces, second memoir, these Tr a n s a c t i o n s, vol. 9 (1908), pp. 79-120, §4.
} 
The directrix of the second kind, $d^{\prime}$, passes through $y$ and through the point

$$
t=y_{u v}-\frac{b_{v}}{2 b} y_{u}-\frac{a_{u}^{\prime}}{2 a^{\prime}} y_{v} .
$$

The directrix congruence of the first kind, $D$, which is formed of the directrices of the first kind, and the directrix congruence of the second kind, $D^{\prime}$, which is formed of the directrices of the second kind, are therefore reciprocal congruences, in virtue of equations (6) and (7).

It is possible to characterize the directrix congruences geometrically without reference to the osculating linear complexes of the asymptotic curves. In fact, let $\Gamma$ and $\Gamma^{\prime}$ be any two congruences reciprocal to each other. The $\Gamma$-curves and $\Gamma^{\prime}$-curves, i. e., the curves on $S$ which correspond to the developables of $\Gamma$ and $\Gamma^{\prime}$, respectively, are defined by the equations

$$
\begin{gathered}
\left(f+\beta^{2}+\beta_{u}+2 b \alpha\right) d u^{2}+\left(\beta_{v}-\alpha_{u}\right) d u d v \\
-\left(g+\alpha^{2}+\alpha_{v}+2 a^{\prime} \beta\right) d v^{2}=0, \\
\left(f+\beta^{2}+\beta_{u}-2 b \alpha+2 b_{v}\right) d u^{2}+\left(\beta_{v}-\alpha_{u}\right) d u d v \\
-\left(g+\alpha^{2}+\alpha_{v}-2 a^{\prime} \beta+2 a_{u}^{\prime}\right) d v^{2}=0 .
\end{gathered}
$$

These two nets of curves coincide if and only if the coefficients of equations (9) and (11) are proportional, i. e., if and only if

$$
\alpha=b_{v} / 2 b, \quad \beta=a_{u}^{\prime} / 2 a^{\prime} .
$$

But then the congruences $\Gamma$ and $\Gamma^{\prime}$ are the directrix congruences, so that we may state the following geometric characterization.

The directrix congruences of the first and second kinds are completely characterized by the following properties:

1. They are reciprocal congruences.

2. Their developables correspond to the same net of curves on the surface $S$.

The second part of this theorem, viz., that the developables of the directrix congruences correspond to the same net on $S$, was discovered by Wilczynski. He calls this net of curves the directrix curves of the surface.

We have of course implicitly assumed that neither $b$ nor $a^{\prime}$ is zero. If $b$ is identically zero, the curves $C_{u}$ (v= const. ) are straight lines, and if $a^{\prime}$ is identically zero the curves $C_{v}(u=$ const. $)$ are straight lines, i. e., in either case the surface is a skew ruled surface. If the surface $S$ is ruled, there is no longer a unique pair of reciprocal congruences whose developables correspond to each other. In fact, if $b=0$, for instance, any pair of reciprocal congruences $\Gamma$ and $\Gamma^{\prime}$ for which $\beta=a_{u}^{\prime} / 2 a^{\prime}$ will have their developables corresponding to the same net on $S$, no matter what the value of $\alpha$ may be. A substitute for the directrix congruences in this case has been furnished by 
Sullivan,* who calls his new congruences the scroll directrix congruences of the first and second kinds. If, for instance, the curves $C_{u}$ are the straight line generators, the scroll directrix congruences are obtained by putting

$$
\alpha=-a_{v}^{\prime} / 4 a^{\prime}, \quad \beta=a_{u}^{\prime} / 2 a^{\prime},
$$

and are in fact reciprocal congruences. Their developables correspond to each other, but they are not the only pair of reciprocal congruences for which this happens. A further characterization is therefore necessary, and we shall supply it later on.

There still remains the case in which both $b$ and $a^{\prime}$ are zero. Both families of asymptotics are then straight lines, so that the surface is a quadric. Inspection of equations (9) and (11) will show that in this case, and only in this case, will the developables correspond to each other for every pair of reciprocal congruences. On a quadric, and only on a quadric, will the curves corresponding to the developables of any congruence $\Gamma$ whatever coincide with the curves corresponding to the developables of the congruence $\Gamma^{\prime}$ which is reciprocal to $\Gamma$.

\section{Some general properties of RECIPRoCAL CONGRUENCES}

We have seen that the directrix congruences of the first and second kinds constitute a pair of reciprocal congruences. A beautiful theorem, which Wilczynski proved for the directrix congruences, and Sullivan for the scroll directrix congruences, will now be proved to be true also for any pair of reciprocal congruences.

The tangents to the two $\Gamma$-curves at a point $y$ of the surface are the lines joining the point $y$ to the two points

$$
y_{u}+\left(\frac{d v}{d u}\right)_{1} y_{v}, \quad y_{u}+\left(\frac{d v}{d u}\right)_{2} y_{v},
$$

where $(d v / d u)_{1}$ and $(d v / d u)_{2}$ are the two roots of equation (9) regarded as a quadratic in $d v / d u$. Let $t_{1}$ and $t_{2}$ denote these two tangents. The direction conjugate to $t_{1}$, and the direction conjugate to $t_{2}$, are obtained by joining the point $y$ to the points

$$
y_{u}-\left(\frac{d v}{d u}\right)_{1} y_{v}, \quad y_{u}-\left(\frac{d v}{d u}\right)_{2} y_{v},
$$

respectively. These new directions we may call the reflected $\Gamma$-tangents, because they are obtained from the $\Gamma$-tangents by harmonic reflection of the latter in the pair of asymptotic tangents. The totality of reflected $\Gamma$-tangents constitute the tangents to the curves of a new net on the surface, which we shall call the reflected $\Gamma$-curves, and which are determined by the differential

*C. T. Sullivan, Scroll directrix curves, these Transactions, vol. 16 (1915), pp. 199-214. 
equation

$$
\begin{aligned}
\left(f+\beta^{2}+\beta_{u}+2 b \alpha\right) d u^{2}-\left(\beta_{v}-\alpha_{u}\right) d u d v & -\left(g+\alpha^{2}+\alpha_{v}+2 a^{\prime} \beta\right) d v^{2}=0 .
\end{aligned}
$$

This equation differs from equation (9) only in the sign of the middle term, because its roots are to be the negatives of the roots of equation (9). But equation (21), considered as a quadratic in $d v / d u$, is the same as equation (14), whose roots $\lambda_{1}$ and $\lambda_{2}$ determine the focal points of the line $l$. We may therefore state the

ThEOREм. The reflected $\Gamma$-tangents intersect the corresponding line $l$ of the congruence $\Gamma$ in the focal points of that line.

This theorem affords excellent evidence that an interesting result may be derived from a trivial one. The theorem is, in fact, a dualization of an obvious situation. By polar reciprocation, the points of a curve $C$ on the surface $S$ go into the tangent planes to a surface $S_{1}$ along a curve $C_{1}$. The tangents of $C$, however, do not correspond to the tangents of $C_{1}$, but to the directions conjugate to the tangents of $C_{1}$. Therefore the dual of the points of a $\Gamma$ curve on $S$ consists of the tangent planes of $S_{1}$ along a $\Gamma_{1}^{\prime}$-curve on $S_{1}$, so that a $\Gamma$-tangent on $S$ corresponds to the conjugate of a $\Gamma_{1}^{\prime}$-tangent on $S_{1}$. But a reflected $\Gamma$-tangent on $S$ goes over into a $\Gamma_{1}^{\prime}$-tangent on $S_{1}$. Moreover, the focal points of a line $l$ of the congruence $\Gamma^{\prime}$ correspond to the focal planes of a line of the corresponding congruence $\Gamma_{1}^{\prime}$. Therefore the lines joining the point $y$ to the focal points of $l$ correspond to $\Gamma_{1}^{\prime}$-tangents on $S_{1}$, so that the theorem in question is the dual of the following trivial statement: the focal planes of a line $l_{1}^{\prime}$ of the congruence $\Gamma_{1}^{\prime}$ intersect the corresponding tangent plane of $S_{1}$ in two lines which coincide with the two $\Gamma_{1}^{\prime}$-tangents of $S_{1}$.

This geometric proof enables us, moreover, to state the theorem more precisely as follows: each reflected $\Gamma$-tangent intersects the corresponding line $i$ of the congruence $\Gamma$ in the focal point of $l$ which corresponds to it.

A one-parameter family of lines $l^{\prime}$ of the congruence $\Gamma^{\prime}$ form a ruled surface. Let us call the ruled surfaces which correspond to the asymptotics of $S$ the asymptotic ruled surfaces of the congruence $\Gamma^{\prime}$, and denote by $R^{(u)}$ and $R^{(v)}$ those which correspond respectively to the curves $C_{u}(v=$ const. $)$ and $C_{v}(u=$ const. $)$ on $S$. Let us consider the ruled surface $R^{(u)}$. The plane determined by the line $l^{\prime}$ and the tangent at $y$ to the curve $C_{v}$ contains a generator of $R^{(u)}$, viz. $l^{\prime}$. If, then, $R^{(u)}$ is not developable, the said plane, which is determined by the points $y, y_{v}, z$, is tangent to the ruled surface $R^{(u)}$ at a definite point of $l^{\prime}$, whose coördinates we proceed to determine. The point sought must be defined by an expression of the form

$$
\zeta_{1}=z+\omega y,
$$

where $\omega$ is to be determined so that the plane $y y_{v} z$ contains the point $\left(\zeta_{1}\right)_{u}$. 
Differentiating the expression for $\zeta_{1}$, and taking the value for $z_{u}$ from equations (10), we obtain

$$
\begin{aligned}
\left(\zeta_{1}\right)_{u}= & -\beta y_{u v}+\left(4 a^{\prime} b-\alpha_{u}+\omega\right) y_{u}-\left(f+2 b_{v}-2 b \alpha+\beta_{u}\right) y_{v} \\
& +\left(2 b g-f_{v}+f \alpha+\omega_{u}\right) y \\
= & -\beta z+\left(4 a^{\prime} b-\alpha_{u}-\alpha \beta+\omega\right) y_{u} \\
& -\left(f+2 b_{v}-2 b \alpha+\beta_{u}+\beta^{2}\right) y_{v}+\left(2 b g-f_{v}+f \alpha+\omega_{u}\right) y .
\end{aligned}
$$

The point $\left(\zeta_{1}\right)_{u}$ therefore lies in the plane of $y, y_{v}, z$ if and only if the coefficient of $y_{u}$ in the above expression is zero, i. e.,

$$
\omega=\alpha \beta+\alpha_{u}-4 a^{\prime} b .
$$

The asymptotic ruled surface $R^{(u)}$ of the congruence $\Gamma^{\prime}$ is touched in the point

$$
\zeta_{1}=z-\left(4 a^{\prime} b-\alpha \beta-\alpha_{u}\right) y
$$

by the plane of the line $l^{\prime}$ and the corresponding tangent of the curve $C_{v}(u=c o n s t$.$) .$

Similarly, the plane determined by the line $l^{\prime}$ and the tangent of the curve $C_{u}$ touches the asymptotic ruled surface $R^{(v)}$ of the congruence $\Gamma^{\prime}$ in the point

$$
\zeta_{2}=z-\left(4 a^{\prime} b-\alpha \beta-\beta_{v}\right) y .
$$

The harmonic conjugate of the point $y$ with respect to $\zeta_{1}$ and $\zeta_{2}$ is the point

$$
\tau=z-\frac{1}{2}\left(8 a^{\prime} b-2 \alpha \beta-\alpha_{u}-\beta_{v}\right) y,
$$

which is precisely the same point as that defined by equation (17). Therefore, the harmonic conjugate of $y$ with respect to $\zeta_{1}$ and $\zeta_{2}$ coincides with the harmonic conjugate of $y$ with respect to the focal points of the line $l^{\prime}$.

Other interesting points on the line $l^{\prime}$ may also be determined. The totality of points $\rho$ form a surface $S_{\rho}$, and the point $\rho_{v}$ is on the tangent to a curve $u=$ const. on $S_{\rho}$. From the geometric definition of the point $\rho$, it is not very difficult to see that the line $\rho \rho_{v}$ intersects the line $l^{\prime}$. In fact, from the second of equations (8) we find that

$$
\rho_{v}-\alpha \rho=y_{u v}-\alpha y_{u}-\beta y_{v}+\left(\alpha \beta-\beta_{v}\right) y=z-\left(\beta_{v}-\alpha \beta\right) y .
$$

The point represented by the left-hand member of this last equation lies on the line $\rho \rho_{v}$, and the point represented by the right-hand member lies on the line $y z$; the points coincide, so the two lines in question intersect. The line $l^{\prime}$ is intersected in the point

$$
\eta_{1}=z-\left(\beta_{v}-\alpha \beta\right) y
$$

by the corresponding tangent to the curve $u=$ const. on the surface $S_{\rho}$.

Similarly, the line $l^{\prime}$ is intersected in the point

$$
\eta_{2}=z-\left(\alpha_{u}-\alpha \beta\right) y
$$

by the corresponding tangent to the curve $v=$ const. on the surface $S_{\sigma}$.

Trans. Am. Math. Soc. 7 
Use will be made subsequently of the various points defined in the present section. We shall, in particular, see how important classes of congruences are obtained when the relations between these points are specialized; a thorough study of the possible interrelations will not be attempted, however, although such a study would undoubtedly lead to interesting results.

\section{The osculating QUADRIC, AND its CONNECTION With RECIPROCAL CONGRUENCES. The CANONICAL QUADRIC}

The four points $y, y_{u}, y_{v}, y_{u v}$ are not coplanar, since by supposition the parametric net on the surface $S$ is not conjugate. Therefore these four points will serve as a non-degenerate local tetrahedron of reference. Any point $X$ in space will be defined as a linear combination of $y, y_{u}, y_{v}, y_{u v}$, say by

$$
X=x_{1} y+x_{2} y_{u}+x_{3} y_{v}+x_{4} y_{u v} .
$$

By choosing properly the unit point of a system of coördinates referred to the tetrahedron with its vertices at $y, y_{u}, y_{v}, y_{u v}$, we may cause the coördinates of the point $X$ to become $x_{1}, x_{2}, x_{3}, x_{4}$ in this new system. Thus, referred to the new tetrahedron, the point $z=y_{u v}-\alpha y_{u}-\beta y_{v}$ has coördinates $(0,-\alpha,-\beta, 1)$.

The tetrahedron of reference just introduced is that which is used by Wilczynski.* Referred to this tetrahedron, the equation of the osculating quadric of the surface at $y$ is found to be

$$
x_{1} x_{4}-x_{2} x_{3}+2 a^{\prime} b x_{4}^{2}=0 .
$$

His derivation of this equation is made to depend upon the formulas derived by him in connection with the general theory of ruled surfaces. $\dagger$ The following independent method for establishing the equation is based on first principles, and may therefore be of sufficient interest to warrant its inclusion here.

Let $R^{(u)}$ represent the ruled surface formed by the tangents to the curves $C_{u}$ at the points where they meet a fixed curve $C_{v}$. Any point $Y$ on this ruled surface will then be given by the expression

$$
Y=y_{u}+u y \text {. }
$$

Since $u$ is fixed, $Y$ is a function of the two independent parameters $v, w$. We seek now the differential equation of the curved asymptotics of the surface $R^{(u)}$. We have

$$
\begin{gathered}
Y_{v}=y_{u v}+w y_{v}, \quad Y_{w}=y, \quad Y_{w w}=0, \\
Y_{v w}=y_{v}, \quad Y_{v v}=y_{u v v}+w y_{v v} .
\end{gathered}
$$

* He uses the letters $z, \rho, \sigma$ to denote the points $y_{u}, y_{v}, y_{u v}$ respectively. Our change of notation need cause no confusion.

† Cf. the second memoir on curved surfaces, these Trans a ction s, vol. 9 (1908), pp. 81-82. 
Differentiation of the second of equations (4) gives the expression for $y_{u v v}$, so that

$$
Y_{v v}=-\left(g+2 a_{u}^{\prime}+2 a^{\prime} w\right) y_{u}+4 a^{\prime} b y_{v}+\left(2 a^{\prime} f-g_{u}-g w\right) y .
$$

We may now obtain the differential equation which defines the asymptotic net on $R^{(u)}$ in the usual way; we need only calculate the four-rowed determinants which are the coefficients of the second fundamental form for the ruled surface.* The required differential equation is without difficulty found to be

$$
2 a^{\prime} b d v^{2}+d v d w=0,
$$

so that the curved asymptotics on $R^{(u)}$ are given by the differential equation

$$
2 a^{\prime} b d v+d w=0 .
$$

We have already fixed the value of $u$; let us now confine our attention to a particular generator $g$ of the ruled surface $R^{(u)}$, viz., the one corresponding to the point $y$. Then $v$ is to be regarded as fixed also. Now, if $g^{\prime}, g^{\prime \prime}$ be two other generators of $R^{(u)}$, the three generators $g, g^{\prime}, g^{\prime \prime}$ determine a quadric, and as $g^{\prime}$ and $g^{\prime \prime}$ both approach $g$ the quadric approaches a limiting quadric, which is the osculating quadric of $R^{(u)}$ along $g$. It is obvious geometrically, and moreover well known, that this quadric has as one set of rulings the asymptotic tangents to $R^{(u)}$ along $g$, in the direction of the curved asymptotics. For these directions along $g$ we have found $d w / d v=-2 a^{\prime} b$, so that at the point $Y$ on $g$ this direction is obtained by joining $Y$ to the point $Y_{v}-2 a^{\prime} b Y_{w}$. In other words, any point $X$ of the osculating quadric of $R^{(u)}$ is given by the expression

$$
X=Y_{v}-2 a^{\prime} b Y_{w}+\lambda Y=y_{u v}+\lambda y_{u}+w y_{v}+\left(w \lambda-2 a^{\prime} b\right) y .
$$

In the right-hand member, $u$ and $v$ are of course to be regarded as fixed, while $w$ and $\lambda$ are the two parameters which determine the point $X$ of the osculating quadric.

Referred to the tetrahedron $y y_{u} y_{v} y_{u v}$, the point $X$ has coördinates $x_{1}$, $x_{2}, x_{3}, x_{4}$ given by

$$
x_{1}=w \lambda-2 a^{\prime} b, \quad x_{2}=\lambda, \quad x_{3}=w, \quad x_{4}=1 .
$$

These are the parametric equations of the quadric; eliminating the parameters $w, \lambda$, and making the resulting equation homogeneous in the standard way, we finally obtain as the equation of the osculating quadric of $R^{(u)}$ along the

* These determinants are

$$
D=\left|Y_{v v}, Y_{v}, Y_{w}, Y\right|, \quad D^{\prime}=\left|Y_{v w}, Y_{v}, Y_{w}, Y\right|, \quad D^{\prime \prime}=\left|Y_{w w}, Y_{v}, Y_{w}, Y\right|,
$$
and the differential equation of the asymptotics is $D d v^{2}+2 D^{\prime} d v d w+D^{\prime \prime} d w^{2}=0$. 
generator $g$ :

$$
x_{1} x_{4}-x_{2} x_{3}+2 a^{\prime} b x_{4}^{2}=0 .
$$

The symmetry of this equation shows that the same quadric osculates also the ruled surface $R^{(v)}$, so that it may properly be called the osculating quadric of the surface $S$ at the point $y$.

It may be proved without difficulty that two lines $l$ and $l^{\prime}$, which according to our geometric definition are reciprocal to each other, are in fact reciprocal polars with respect to the osculating quadric. This furnishes another justification for our previous designation. Geometrically, the situation is almost evident. The ruled surface $R^{(u)}$ of asymptotic tangents has at the point $\rho$ the same tangent plane as the osculating quadric. In other words, the line $l$ intersects the quadric at $\rho$ and $\sigma$, and the tangent planes to the quadric at these points meet in the line $l^{\prime}$, which is therefore the reciprocal polar of $l$ with respect to the quadric.

The line $l^{\prime}$ meets the quadric in two points, one of which is $y$ and the other of which is of the form $z+\omega y$. Referred to the tetrahedron $y y_{u} y_{v} y_{u v}$ the point $z+\omega y$ has the coördinates $(\omega,-\alpha,-\beta, 1)$. This point lies on the osculating quadric if and only if its coördinates satisfy the equation of the quadric, (27), i. e., if and only if $\omega-\alpha \beta+2 a^{\prime} b=0$ : This gives the value of $\omega$, and we see that the line $l^{\prime}$ cuts the osculating quadric in the points $y$ and

$$
Z=z-\left(2 a^{\prime} b-\alpha \beta\right) y .
$$

This point $Z$ bears an interesting relation to the points

$$
\eta_{1}=z-\left(\beta_{v}-\alpha \beta\right) y, \quad \zeta_{2}=z-\left(4 a^{\prime} b-\alpha \beta-\beta_{v}\right) y
$$

of the preceding section. In fact, the harmonic conjugate of $y$ with respect to these points is the point $Z$. The points $\eta_{1}$ and $\zeta_{2}$ separate harmonically the two points in which the line $l^{\prime}$ cuts the osculating quadric. A similar theorem subsists for the points $\eta_{2}$ and $\zeta_{1}$. We may say, then, that the points in which the line $l^{\prime}$ cuts the osculating quadric are the double points of the involution determined by the two pairs of points $\eta_{1}, \zeta_{2}$ and $\eta_{2}, \zeta_{1}$.

Another very important quadric is the one whose equation is

$$
x_{1} x_{4}-x_{2} x_{3}=0 .
$$

It is called the canonical quadric, ${ }^{*}$ and plays such an important part in the general theory of surfaces that its characterization by elementary methods would be desirable. It is one of the pencil of quadrics

$$
x_{1} x_{4}-x_{2} x_{3}+\lambda x_{4}^{2}=0,
$$

* Cf. E. J. Wilczynski, Curved Surfaces, second memoir, these $\mathrm{Tr}$ a n s a t i o n s, vol. 9 (1908), p. 112. 
all of which are completely defined-not individually, but as members of the pencil-by the property, that they are tangent to the osculating hyperboloid all along the corresponding asymptotic tangents of the surface $S . *$ The canonical quadric itself is then completely determined if in addition it be required to pass through the point $(\alpha \beta,-\alpha,-\beta, 1)$, i. e., the point $z+\alpha \beta y$. A geometric definition of this point must therefore still be given, in order to complete the geometric characterization of the canonical quadric. It seems to us highly desirable to obtain this definition independently of Wilczynski's canonical cubic. This we have been unable to do; the points $Z, \eta_{1}, \eta_{2}, \zeta_{1}$, $\zeta_{2}$ of this and the preceding sections obviously have a close connection with the desired point, but do not suffice for its determination.

\section{Congruences Conjugate and harmonic to a surface}

A conjugate net on a surface is called by Guichard a réseau. $\dagger$ If a surface is referred to a réseau, and if the developables of a congruence $\Gamma^{\prime}$ cut the surface in these parametric curves, he says that the congruence and the réseau are conjugate to each other. $\ddagger$ If the developables of any congruence $\Gamma^{\prime}$ cut the surface $S$ in a conjugate net, we shall say that the congruence $\Gamma^{\prime}$ is conjugate to the surface $S$, or that the congruence and surface are conjugate to each other, no matter what the parametric curves of the surface may be.

We shall borrow also another term used by Guichard. We shall say that a congruence $\Gamma$ is harmonic to a surface $S$, or that the congruence and surface are harmonic to each other, if its developables correspond to a conjugate net on $S$. Our definition will presently be reconciled with that of Guichard; the two definitions will, by means of an important theorem, be shown to lead to exactly the same class of congruences $\Gamma$.

Any net of curves on the surface $S$ may be defined by means of a quadratic differential equation of the form

$$
P(u, v) d u^{2}+2 Q(u, v) d u d v+R(u, v) d v^{2}=0,
$$

for which $Q^{2}-P R \neq 0$. If the surface is referred to its asymptotic curves, then obviously the net is conjugate if and only if $Q=0$.

A glance at equations (9) and (11) will show that the $\Gamma$-curves, and likewise the $\Gamma^{\prime}$-curves, form a conjugate net if and only if $\alpha_{u}-\beta_{v}=0$. Of course it is here assumed that neither $P$ nor $R$ is zero in either differential equation. If, for instance, $P$ were equal to zero, the asymptotics $C_{u}$ ( $v=$ const. ) would be

\footnotetext{
* The additional property stated by Wilczynski, viz., that they have contact of the second order with $S$, is redundant.

†C. Guichard, Sur les systèmes orthogonaux et les systemes cycliques. A n n ale s de l'é c o le n or ma le, 3 série, v. 14 (1897), pp. 467-516. As is well known, he also applies the name to the generalization of conjugate nets in hyperspace.

$\ddagger$ Loc. cit., p. 478.
} 
a family of $\Gamma$-curves or of $\Gamma^{\prime}$-curves, according as $P$ is the first coefficient of (9) or of (11). If one family of a net consists of asymptotics, the other family can not be conjugate to the first. Of course if $Q$ is zero and either $P$ or $R$ also zero, then the two families of the net conicide; i. e., the differential equation does not define a net at all. So, recalling our definitions of congruences conjugate and harmonic to a surface, we may state our theorems more carefully as follows:

If the $\Gamma$-curves form a net on the surface $S$, then the congruence $\Gamma$ is harmonic to the surface $S$ if and only if

$$
\alpha_{u}-\beta_{v}=0 .
$$

If the $\Gamma^{\prime}$-curves form a net on the surface $S$, then the congruence $\Gamma^{\prime}$ is conjugate to the surface $S$ if and only if $\alpha_{u}-\beta_{v}=0$.

If both the I'curves and $\Gamma^{\prime}$-curves form nets on the surface $S$, then the congruence $\Gamma$ is harmonic to the surface $S$ if and only if the congruence $\Gamma^{\prime}$ is conjugate to $S$, and conversely.

An important special case is that in which either of the nets of $\Gamma$-curves and $\Gamma^{\prime}$-curves is indeterminate, or in which both are, i. e., when all of the coefficients in either or both of equations (9) and (11) vanish identically. We shall consider this case later. To simplify the statements of theorems for the present, let us suppose that the discriminants of both (9) and (11) are different from zero, i. e., that the $\Gamma$-curves and $\Gamma^{\prime}$-curves actually form nets.

Several geometric criteria that congruences be conjugate or harmonic to a surface may be deduced from some of our previous work. Equations (23), (24), (25), and (26) show that the points $\zeta_{1}$ and $\zeta_{2}$ coincide, as do also the points $\eta_{1}$ and $\eta_{2}$, if and only if the congruence $\Gamma$ is harmonic, and the congruence $\Gamma^{\prime}$ conjugate, to the surface $S$.

A more important criterion for the above situation is obtained as follows. A comparison of equations (9) and (14) will show that if $\alpha_{u}-\beta_{v}=0$ the quadratic in $\lambda$ is the same as the quadratic (9) in $d v / d u$. There is one other case, however, in which the two quadratics give the same roots, viz., when

$$
f+\beta^{2}+\beta_{u}+2 b \alpha=0, \quad g+\alpha^{2}+\alpha_{v}+2 a^{\prime} \beta=0,
$$

i. e., the $\Gamma$-curves coincide with the asymptotic net. In both of the cases just mentioned, the tangents to the $\Gamma$-curves at $y$ meet the line $l$ in the focal points of $l$. We may state our result as follows. The tangents to the $\Gamma$ curves at a point of a surface meet the corresponding line $l$ in the focal points of $l$ if and only if the congruence $\Gamma$ has one of the following properties (it cannot have both):

1. It is harmonic to the surface.

2. Its developables correspond to the asymptotic curves of the surface.

The first case is of course the more important of the two. In fact, the 
designation harmonic is used by Guichard in essentially this sense, i. e., a congruence $\Gamma$ is said to be harmonic to a réseau if each line $l$ of the congruence is met in its focal points by the tangents to the curves of the réseau constructed at the corresponding point of the surface.* The theorem which we have stated above, however, shows the desirability of our definition, if only for the sake of symmetry in formulation.

A closer examination of the situation, however, will enable us to state our theorem so as to distinguish between the two cases arising therein, and thereby obtain a refinement of Guichard's definition. Let $t_{1}$ and $t_{2}$ be the $\Gamma$-tangents at the point $y$, and $\Phi_{1}$ and $\Phi_{2}$ the corresponding focal points of the line $l$. Then the theorem of page 94 shows that the $\Gamma$-curves form a conjugate net if and only if $t_{1}$ meets $l$ in $\Phi_{2}$, and $t_{2}$ meets $l$ in $\Phi_{1}$. We may therefore state our result as follows:

The congruence $\Gamma$ is harmonic to the surface if and only if the $\Gamma$-tangents at each point of the surface meet the corresponding line $l$ of the congruence in its focal points, each $\Gamma$-tangent having on it the focal point which corresponds to the other $\Gamma$-tangent.

We may also state the theorem, that the $\Gamma$-curves coincide with the asymptotic net on the surface if and only if the $\Gamma$-tangent meet the corresponding line l in its focal points, each $\Gamma$-tangent having on it the focal point which corresponds to it.

The characteristic condition for congruences harmonic and conjugate to a surface, viz., $\alpha_{u}-\beta_{v}=0$, shows that in this case there exists a function $\theta$ such that

$$
\alpha=\frac{\theta_{v}}{\theta}, \quad \beta=\frac{\theta_{u}}{\theta} .
$$

So we have the theorem, that any congruence $\Gamma$ harmonic to a surface consists of lines joining the points

$$
\rho=\theta y_{u}-\theta_{u} y, \quad \sigma=\theta y_{v}-\theta_{v} y,
$$

where $\theta$ is any function of $(u, v)$, and conversely.

A congruence $\Gamma^{\prime}$ conjugate to a surface consists of the lines joining each point $y$ to the corresponding point

$$
z=\theta y_{u v}-\theta_{v} y_{u}-\theta_{u} y_{v},
$$

where $\theta$ is any function of $(u, v)$, and conversely.

For congruences $\Gamma$ and $\Gamma^{\prime}$ which are reciprocal to each other, the functions $\theta$ in (30) and (31) are the same.

We may remark here that, according to the above considerations, if the asymptotic curves of a surface be known, the determination of all the congruences conjugate and harmonic to the surface requires no integration, and all of these

* Guichard, loc. cit., p. 483. 
congruences may be defined explicitly by means of formulas (30) and (31). However, if a particular conjugate net is designated in advance to serve as the net of $\Gamma$-curves, say, then the determination of all congruences $\Gamma$ whose developables correspond to the curves of this given net requires further integrations, the precise nature of which we shall not discuss here.

Formulas (30) and (31) still serve to define a pair of reciprocal congruences conjugate and harmonic to $S$, if the system of differential equations of which $S$ is an integral surface be given in the more general form

$$
\begin{aligned}
y_{u u}+2 a y_{u}+2 b y_{v}+c y & =0, \\
y_{v v}+2 a^{\prime} y_{u}+2 b^{\prime} y_{v}+c^{\prime} y & =0,
\end{aligned}
$$

instead of in the canonical form (4). In fact, according to the remark made towards the end of $\S 1$, page 87 , formulas (6) and (7) for the points $\rho, \sigma$, $z$ subsist if the differential equations are in the general form (2). Therefore, in virtue of equations (4a), we observe that if the differential equations are given in the form (2), the points $\rho, \sigma, z$ given by equations (6) and (7) define a pair of reciprocal congruences, whose $\Gamma$-and $\Gamma^{\prime}$-curves are defined by the differential equations (9) and (11), in which $f$ and $g$ have been replaced by their equivalent expressions (4a). It follows at once that equations (30) and (31) define also in this case a pair of reciprocal congruences conjugate and harmonic to the surface, as we stated above.

Analytically, the simplest pair of reciprocal congruences, one of them harmonic and the other conjugate to the surface, are those for which $\alpha=0$, $\beta=0$. The line $l$ joins the points $y_{u}, y_{v}$, and the line $l^{\prime}$ the points $y, y_{u v}$. It is easily shown that this really yields the most general case, as follows. Since the coördinates $y^{(k)}$ of a point of the surface are homogeneous, all points of the surface will remain fixed if the dependent variable be transformed by means of the equation

$$
y=\theta(u, v) \bar{y} .
$$

If, now, we write instead of equations (30)

$$
\rho=\frac{\theta y_{u}-\theta_{u} y}{\theta^{2}}, \quad \sigma=\frac{\theta y_{v}-\theta_{v} y}{\theta^{2}}
$$

which yield the same points as before on account of the homogeneity of our coördinates, and instead of equation (31)

$$
\bar{z}=\frac{\theta y_{u v}-\theta_{v} y_{u}-\theta_{u} y_{v}-\theta_{u v} y}{\theta^{2}}
$$

which is a new point on the line $l^{\prime}$, or $y z$, we find that in virtue of (32)

$$
\rho=\bar{y}_{u}, \quad \sigma=\bar{y}_{v}, \quad \bar{z}=\bar{y}_{u v} .
$$


But the system of differential equations (2) becomes under the transformation (32) a new system of the same form,

where

$$
\begin{aligned}
\bar{y}_{u u}+2 \bar{a} \bar{y}_{u}+2 \bar{b} \bar{y}_{v}+\bar{c} \bar{y} & =0, \\
\bar{y}_{v v}+2 \bar{a}^{\prime} \bar{y}_{u}+2 \bar{b}^{\prime} \bar{y}_{v}+\bar{c}^{\prime} \bar{y} & =0,
\end{aligned}
$$

$$
\bar{a}=a+\frac{\theta_{u}}{\theta}, \quad \bar{b}=b, \quad \bar{c}=\frac{\theta_{u u}+2 a \theta_{u}+2 b \theta_{v}+c \theta}{\theta},
$$

$$
\bar{a}^{\prime}=a^{\prime}, \quad \bar{b}^{\prime}=b^{\prime}+\frac{\theta_{v}}{\theta}, \quad \bar{c}^{\prime}=\frac{\theta_{v v}+2 a^{\prime} \theta_{u}+2 b^{\prime} \theta_{v}+c^{\prime} \theta}{\theta} .
$$

With respect to system (34), therefore, the quantities (33) actually define a pair of reciprocal congruences, one harmonic and the other conjugate to the surface $S$, and, since these coincide with the congruences defined by equations (30) and (31), they are the most general pair having these properties.

The importance of congruences conjugate and harmonic to a surface is well established by the part they play in the transformation theory of surfaces. It is hoped that the formulas which have been derived in the present section may provide a new approach to this general theory. An interesting problem which naturally suggests itself is the determination and investigation of the congruences which are conjugate to two surfaces and which in addition cause the asymptotic nets on the two surfaces to correspond. These congruences afford a generalization of the $W$-congruences, and suggest a certain transformation of a given surface which we may call a transformation $W$. The determination of all surfaces into which a given congruence conjugate to a given surface $S$ will transform $S$ so that the two surfaces are in the relation of a transformation $W$ requires the integration of a partial differential equation of the second order. Particularly interesting would be of course the transformations $W$ for which the surface $S$ is transformed into its point conjugate with respect to the congruence of the transformation.* The determination of all congruences which afford such transformations $W$ of a given surface requires the integration of a certain partial differential equation of the fourth order.

\section{Congruences Whose $\Gamma$-Curves or $\Gamma^{\prime}$-Curves are indeterminate}

The condition $\alpha_{u}-\beta_{v}=0$ may be replaced, as we have seen, by the equations

$$
\alpha=\frac{\theta_{v}}{\theta}, \quad \beta=\frac{\theta_{u}}{\theta},
$$

where $\theta$ is some function of $(u, v)$ which is nowhere zero. The differential

* See the end of $\S 4$ for the definition of point conjugate of a surface with respect to a congruence. 
equations for the $\Gamma$-curves and $\Gamma^{\prime}$-curves take on a remarkable form in this case. Substituting the values of $\alpha$ and $\beta$ in equations (9) and (11), we find for the differential equation of the $\Gamma$-curves

$$
\left(\theta_{u u}+2 b \theta_{v}+f \theta\right) d u^{2}-\left(\theta_{v v}+2 a^{\prime} \theta_{u}+g \theta\right) d v^{2}=0,
$$

and for the differential equation of the $\Gamma^{\prime}$-curves

$$
\left[\theta_{u u}-2 b \theta_{v}+\left(f-2 b_{v}\right) \theta\right] d u^{2}-\left[\theta_{v v}-2 a^{\prime} \theta_{u}+\left(g-2 a_{u}^{\prime}\right) \theta\right] d v^{2}=0 .
$$

The coefficients of this last equation are the differential expressions adjoint to those which constitute the coefficients of equation (35). Moreover, the coefficients of (35) are the left-hand members of the fundamental differential equations (4) with $\theta$ substituted for $y$.

We shall say that the congruence $\Gamma$ has indeterminate $\Gamma$-curves if all the coefficients of equation (9) vanish identically. We may then state the

Theorem. All congruences $\Gamma$ with indeterminate $\Gamma$-curves are generated by the line l joining the points

$$
\rho=\theta y_{u}-\theta_{u} y, \quad \sigma=\theta y_{v}-\theta_{v} y,
$$

where $\theta$ is any non-vanishing solution whatever of the same system of differential equations as is satisfied by the four functions $y$ :

$$
\theta_{u u}+2 b \theta_{v}+f \theta=0, \quad \theta_{v v}+2 a^{\prime} \theta_{u}+g \theta=0 .
$$

All congruences $\Gamma^{\prime}$ with indeterminate $\Gamma^{\prime}$-curves are generated by the line $l^{\prime}$ which joins the points $y$ and

$$
z=\phi y_{u v}-\phi_{v} y_{u}-\phi_{u} y_{v},
$$

where $\phi$ is any non-vanishing solution of the system of differential equations adjoint to the system satisfied by the four functions $y$ :

$$
\begin{aligned}
\phi_{u u}-2 b \phi_{v}+\left(f-2 b_{v}\right) \phi & =0, \\
\phi_{v v}-2 a^{\prime} \phi_{u}+\left(g-2 a_{u}^{\prime}\right) \phi & =0 .
\end{aligned}
$$

Let us first consider the case in which the $\Gamma$-curves are indeterminate. Since system (4) is completely integrable, any solution $\theta$ thereof is linearly expressible, with constant coefficients, in terms of the four functions (1):

$$
\theta=c_{1} y^{(1)}+c_{2} y^{(2)}+c_{3} y^{(3)}+c_{4} y^{(4)} \text {. }
$$

Since $\rho^{(k)}=\theta y_{u}^{(k)}-\theta_{u} y^{(k)}(k=1,2,3,4)$, we see at once that

$$
c_{1} \rho^{(1)}+c_{2} \rho^{(2)}+c_{3} \rho^{(3)}+c_{4} \rho^{(4)}=0 \text {; }
$$

in other words, all the points $\rho$ lie in a plane. Similarly, all the points $\sigma$ lie in the same plane. Therefore the congruence $\Gamma$ consists entirely of lines 
lying in this plane. In fact, any congruence $\Gamma$ with indeterminate $\Gamma$-curves consists of the lines in which an arbitrary fixed plane cuts all the tangent planes of the surface.

This suggests an interesting way of constructing certain congruences $\Gamma^{\prime}$ conjugate to the surface $S$. For, having constructed as just indicated a congruence $\Gamma$ with indeterminate $\Gamma$-curves, and having therefore determined the points $\rho$ and $\sigma$ corresponding to each point $y$ of $S$, construct at $\rho$ and $\sigma$ the planes which are tangent to the ruled surfaces $R^{(u)}$ and $R^{(v)}$ formed by the asymptotic tangents. The line of intersection of these two planes is the line $l^{\prime}$ which generates the required congruence $\Gamma^{\prime}$. This construction requires of course that we be able to construct the tangent to a curve at any point. That the congruence $\Gamma^{\prime}$, which is reciprocal to $\Gamma$, is conjugate to the surface $S$, follows from the fact that $\alpha_{u}-\beta_{v}=0$. We suppose, of course, in speaking of $\Gamma^{\prime}$ as conjugate to the surface, that the $\Gamma^{\prime}$-curves are not indeterminate also.

In like manner, we may prove that if the $\Gamma^{\prime}$-curves are indeterminate, the lines of the congruence $\Gamma^{\prime}$ all pass through a point. This follows at once, however, by the principle of duality. The corresponding reciprocal congruence $\Gamma$ will then be harmonic to the surface $S$.

A very interesting case, which we have excluded in the above discussion, is that in which both the $\Gamma$-curves and the $\Gamma^{\prime}$-curves are indeterminate. In this case, if the surface is not ruled, the congruences $\Gamma$ and $\Gamma^{\prime}$ are the directrix congruences. The special class of surfaces for which the directrix curves are indeterminate has been studied by Wilczynski.*

If the system of differential equations of which $S$ is an integral surface is not in the canonical form (4), but in the more general form (2), the differential equation of the $\Gamma$-curves is obtained from equation (9) by replacing $f$ and $g$ by their values as given by equations (4a). If the $\Gamma$-curves form a conjugate net, then $\alpha$ and $\beta$ may be replaced by $\theta_{v} / \theta$ and $\theta_{u} / \theta$ respectively, and the differential equation of the $\Gamma$-curves becomes

$$
\left(\theta_{u u}+2 a \theta_{u}+2 b \theta_{v}+c \theta\right) d u^{2}-\left(\theta_{v v}+2 a^{\prime} \theta_{u}+2 b^{\prime} \theta_{v}+c^{\prime} \theta\right) d v^{2}=0 .
$$

Similarly, if the $\Gamma^{\prime}$-curves form a conjugate net, their differential equation becomes

$$
\begin{aligned}
{\left[\phi_{u u}+2 a \phi_{u}-2 b \phi_{v}+\left(c+2 b_{v}-4 b b^{\prime}\right) \phi\right] d u^{2} } \\
-\left[\phi_{v v}-2 a^{\prime} \phi_{u}+2 b^{\prime} \phi_{v}+\left(c^{\prime}+2 a_{u}^{\prime}-4 a a^{\prime}\right) \phi\right] d v^{2}=0,
\end{aligned}
$$

if $\alpha$ and $\beta$ are replaced by $\phi_{v} / \phi$ and $\phi_{u} / \phi$, respectively. The coefficients in this last equation are not adjoint, in the ordinary (Lagrange) sense, to the coefficients of equation (39), which are the left-hand members of equations

* E. J. Wilczynski, Über Flächen mit unbestimmten Direktrixkurven, $\mathrm{M}$ a t h e $\mathrm{m}$ a t i s c h e A nn a le n, vol. 76 (1915), pp. 129-160. 
(2). Huwever, the system

$$
\begin{aligned}
\phi_{u u}+2 a \phi_{u}-2 b \phi_{v}+\left(c+2 b_{v}-4 b b^{\prime}\right) \phi & =0, \\
\phi_{v v}-2 a^{\prime} \phi_{u}+2 b^{\prime} \phi_{v}+\left(c^{\prime}+2 a_{u}^{\prime}-4 a a^{\prime}\right) \phi & =0
\end{aligned}
$$

is the geometric adjoint of system (2), in the sense that a surface defined in point coördinates by a fundamental system of solutions of (2) is the same surface as that defined in plane coördinates by a corresponding fundamental system of solutions of (41).* When the system of differential equations is in the canonical form (4), however, the geometric adjoint system coincides with the Lagrange adjoint. We must therefore describe the determination of congruences $\Gamma^{\prime}$ with indeterminate $\Gamma^{\prime}$-curves more carefully as follows. A necessary and sufficient condition that the congruence of lines joining each point $y$ of an integral surface of system (2) to the corresponding point

$$
z=y_{u v}-\alpha y_{u}-\beta y_{v}
$$

have indeterminate $\Gamma^{\prime}$-curves, is that $\alpha=\phi_{v} / \phi, \beta=\phi_{u} / \phi$, where $\phi$ is a nonvanishing solution of system (41), which is the geometric adjoint of system (2).

\section{Planar Nets with equal invariants}

To Koenigs is due the well-known theorem, that a necessary and sufficient condition that a net of curves in a plane have equal Laplace-Darboux invariants is that it be the perspective on that plane of the asymptotic net on a curved surface. We have elsewhere $\dagger$ given another geometric characterization of such planar nets, without finding it necessary to introduce any configuration outside of the plane of the net. It is the purpose of the present section to put this characterization into relation with that of Koenigs, by employing the facts just developed for reciprocal congruences whose $\Gamma^{\prime}$-curves are indeterminate.

Let $N$ be any net of curves in a plane $\pi$, two curves of the net passing through each point $\eta$ of a region of $\pi$. Let $\tau_{1}$ and $\tau_{2}$ be the tangents-supposed to be distinct-to these curves at $\eta$. Any regular one-parameter family of lines in the plane will have an envelope; consequently, the tangents to the curves of one family of $N$, constructed at the points of a fixed curve of the other family, will envelop a curve, so that on the tangent $\tau_{1}$ is determined a point $\rho^{\prime}$, where $\tau_{1}$ touches its envelope, and on $\tau_{2}$ is determined a point $\sigma^{\prime}$, where $\tau_{2}$ touches its envelope. We shall call the line $\rho^{\prime} \sigma^{\prime}$ the ray of the point $\eta$.

* Cf. E. J. Wilczynski, Curved Surfaces, first memoir, these Trans a c ti o n s, vol. 8 (1907), p. 259.

$\dagger \mathrm{G}$. M. Green, Plane nets with equal invariants, A n nals of $\mathrm{M}$ at he matics, vol. 19 (1918), pp. 246-250. 
Let $N^{\prime}$ be another net in $\pi$, and $\tau_{1}^{\prime}, \tau_{2}^{\prime}$ the tangents to the curves of $N^{\prime}$ at $\eta$. As $\eta$ traces the curve of $N^{\prime}$. to which $\tau_{1}^{\prime}$ is tangent, the corresponding rays $\rho^{\prime} \sigma^{\prime}$ envelop a curve; let $\Phi_{1}$ be the point at which $\rho^{\prime} \sigma^{\prime}$ touches this curve. We may call $\Phi_{1}$ the focal point of the ray corresponding to $\tau_{1}^{\prime}$. In the same way we may define a second focal point $\Phi_{2}$ on $\rho^{\prime} \sigma^{\prime}$, corresponding to $\tau_{2}^{\prime}$. Now, in the paper cited, we have shown that there always exists one and only one net $N^{\prime}$ associated with a given net $N$, and having the following congruential property: the harmonic conjugate of $\tau_{1}^{\prime}$ with respect to $\tau_{1}, \tau_{2}$ passes through $\Phi_{1}$, and the harmonic conjugate of $\tau_{2}^{\prime}$ with respect to $\tau_{1}, \tau_{2}$ passes through $\Phi_{2}$. We say that the net $N^{\prime}$ is in this case congruentially associated with the net $N$.

The geometric characterization referred to above is involved in the following theorem. A planar net has equal invariants if and only if at every point $\eta$ the tangents to the curves of the net are separated harmonically by the tangents to the curves of the congruentially associated net, or in other words if and only if the tangents to the curves of the congruentially associated net pass through the focal points of the ray, each of the said tangents having on it that focal point of the ray which corresponds to the other tangent.

Let us return now to the surface $S$, referred to its asymptotic net. From a point $P$ in space, project the surface on a plane $\pi$ which does not contain $P$. Each point $y$ of $S$ goes into a corresponding point $\eta$ of $\pi$, and the asymptotic net on $S$ projects into a net $N$ on $\pi$. By the theorem of Koenigs, the net $N$ has equal invariants; but we shall now show geometrically that the net $N$ has the geometric properties involved in the theorem just stated, thereby proving independently that the perspective of the asymptotic net has equal invariants.

The lines which project the surface $S$ into the plane $\pi$ all pass through the point $P$, so that they form a congruence $\Gamma^{\prime}$ with indeterminate $\Gamma^{\prime}$-curves. The reciprocal congruence $\Gamma$ is therefore harmonic to the surface $S-\mathrm{i}$. e., its developables correspond to a conjugate net on $S$-according to the results of the preceding section.* The $\Gamma$-curves are projected from $P$ into a net $N^{\prime}$ on $\pi$. Since the $\Gamma$-curves form a conjugate net, the tangents $t_{1}^{\prime}, t_{2}^{\prime}$ to the $\Gamma$-curves at $y$ separate harmonically the asymptotis tangents $t_{1}, t_{2}$. Therefore the tangents $\tau_{1}^{\prime}, \tau_{2}^{\prime}$ to the curves of $N^{\prime}$ at $\eta$ separate harmonically the tangents $\tau_{1}, \tau_{2}$ to the curves of $N$. Now, when a developable is projected on a plane, its edge of regression goes into the envelope of the lines into which the generators of the developable are projected. Therefore the focal points $F_{1}$ and $F_{2}$ of the line $l$ of the congruence $\Gamma$ are projected into the focal points $\Phi_{1}$ and $\Phi_{2}$ of the corresponding line $\lambda$ of the plane $\pi$. $\dagger$ It remains to show

* We assume of course that the congruence $\Gamma^{\prime}$ is not the directrix congruence of the second kind, for in that case all the lines of $\Gamma$ would lie in a plane, and all the ruled surfaces of the congruence would be developables.

$\dagger$ That is, the focal points of $\lambda$, corresponding to displacements of $\eta$ along curves of $N^{\prime}$. 
that the line $\lambda$ intersects the tangents $\tau_{1}, \tau_{2}$ of the curves of $N$ in the points $\rho^{\prime}, \sigma^{\prime}$, where $\rho^{\prime}$ is on the envelope of the tangents $\tau_{1}$ constructed along the fixed curve of $N$ to which $\tau_{2}$ is tangent, and $\sigma^{\prime}$ is on the envelope of tangents $\tau_{2}$ constructed in a similar way. This is not difficult to see geometrically. As $y$ moves along the asymptotic $u=$ const. on $S$, the point $\rho$ on the asymptotic tangent in the other direction, viz. $t_{1}$, traces a curve, the tangent to which lies in the plane determined by $t_{1}$ and $l^{\prime}$, according to the geometric definition of reciprocal congruences. But this plane of $t_{1}$ and $l^{\prime}$ is the plane which projects $t_{1}$ into $\tau_{1}$; since this plane is tangent to the curve traced by $\rho$, it is also tangent to the curve traced by the corresponding point $\rho^{\prime}$. In other words, the line $\tau_{1}$ is tangent to the curve traced by $\rho^{\prime}$ as the corresponding point $\eta$ traces the curve of the net $N$ in the direction $\tau_{2}$. The net $N^{\prime}$ therefore bears to the net $N$ essentially the same geometric relation as that which the net of $\Gamma$-curves bears to the asymptotic net of $S$. In fact, the net $N^{\prime}$ is congruentially associated with the net $N$-we now see the reason for this designation-and, since its tangents separate harmonically those of $N$, the net $N$ is proved to have equal invariants, according to our theorem as stated above.

The fact that the perspective, on a plane, of an asymptotic net on a surface is a planar net with equal invariants, may therefore be deduced from our general theorem. The remainder of Koenigs's theorem, to the effect that every planar net with equal invariants is the perspective of some asymptotic net, does not follow easily from our theorem, however; nor could it be expected to, since its proof involves the solution of differential equations which, it is true, are solvable by quadratures.* It is not difficult to see, however, that the use of the properties of reciprocal congruences will enable one to prove our theorem in its entirety, if the whole of Koenigs's theorem be assumed. It should be noted, however, that a direct proof of our complete theorem, as is given in the paper already cited, is much simpler than any that have as yet been devised for the second part of Koenigs's theorem.

\section{The Perspectives of the asymptotic CURVES ON the tangent Plane, AND THEIR FOUR-POINT AND OSCULATING CONICS}

Let $y$ be any point of the surface $S$, and let $\Gamma$ and $\Gamma^{\prime}$ be any pair of reciprocal congruences. Any point of the line $l^{\prime}$ which passes through $y$ is given by an expression of the form

$$
\tau=y_{u v}-\alpha y_{u}-\beta y_{v}+\lambda y,
$$

* Cf. G. Koenigs, Sur les réseaux plans à invariants égaux et les lignes asymptotiques. Comptes Rendus, vol. 94 (1892), pp. 55-57. The quadratures are carried out explicitly by L. P. Eisenhart, A n n a l s of M a the matic s, vol. 18 (1917), pp. 221-225. An analytic proof of the complete theorem of Koenigs is also to be found in a paper by $\mathrm{A}$. $\mathrm{L}$. Nelson, Pal e r m o Rendic on ti, vol. 42 (1916), pp. 251 et seq. 
where $\lambda$ is an arbitrary function of $(u, v)$. We shall, however, confine our attention to a particular point $y$ of the surface, and without loss of generality may suppose the corresponding parametric values to be $u=0, v=0$. Then, for this point, $y$ and its derivatives, as well as $\alpha, \beta, \lambda$, and $\tau$, have fixed values. We shall project the surface $S$, in the neighborhood of $y$, upon the tangent plane at $y$, using the point $\tau$ as center of projection.

Our first object of investigation will be the asymptotic $v=0$ which passes through $y(u=0, v=0)$. A point $Y$ on this asymptotic in the neighborhood of $y$ is given by the Taylor development.*

$$
Y=y+u y_{u}+\frac{1}{2} u^{2} y_{u u}+\frac{1}{6} u^{3} y_{u u u}+\cdots \text {. }
$$

By differentiation of equations (4), and by reason of the complete integrability of this system, any derivative of $y$ is expressible as a unique linear combination of $y, y_{u}, y_{v}, y_{u v}$. Let us write

$$
\frac{\partial^{p+q} y}{\partial u^{p} \partial v^{q}}=\alpha^{(p q)} y+\beta^{(p q)} y_{u}+\gamma^{(p q)} y_{v}+\delta^{(p q)} y_{u v}
$$

Then all of the derivatives of $y$ in (42) may be replaced by linear expressions of this form; in fact, if the substitutions be made in (42), we find without difficulty that

$$
Y=Y_{1} y+Y_{2} y_{u}+Y_{3} y_{v}+Y_{4} y_{u v}
$$

where

$$
\begin{aligned}
& Y_{1}=1-\frac{1}{2} f u^{2}+\frac{1}{6} \alpha^{(30)} u^{3}+\frac{1}{2} \frac{1}{4} \alpha^{(40)} u^{4}+\cdots, \\
& Y_{2}=u+\frac{1}{6} \beta^{(30)} u^{3}+\frac{1}{24} \beta^{(40)} u^{4}+\cdots, \\
& Y_{3}=-b u^{2}+\frac{1}{6} \gamma^{(30)} u^{3}+\frac{1}{2} \gamma^{(40)} u^{4}+\cdots, \\
& Y_{4}=\frac{1}{6} \delta^{(30)} u^{3}+\frac{1}{24} \delta^{(40)} u^{4}+\cdots .
\end{aligned}
$$

We have in these equations already replaced $\alpha^{(20)}, \beta^{(20)}, \gamma^{(20)}, \delta^{(20)}$ by their values

$$
\alpha^{(20)}=-f, \quad \beta^{(20)}=\delta^{(20)}=0, \quad \gamma^{(20)}=-2 b
$$

taken directly from equations (4).

As in $\S 7$, we may choose as a new tetrahedron of reference one with the points $y, y_{u}, y_{v}, y_{u v}$ as vertices, so that, with a suitably chosen unit point, the coördinates of the point $Y$ referred to this tetrahedron will be $Y_{1}, Y_{2}$, $Y_{3}, Y_{4}$. We shall, however, choose for a local tretahedron the one with vertices at $y, \rho, \sigma, \tau$, where

$$
\rho=y_{u}-\beta y, \quad \sigma=y_{v}-\alpha y, \quad \tau=y_{u v}-\alpha y_{u}-\beta y_{v}+\lambda y .
$$

We may replace $y_{u}, y_{v}$, and $y_{u v}$ everywhere by their expressions in terms of

\footnotetext{
* It is, of course, unnecessary to suppose the functions involved to be analytic. For our purposes, it will be sufficient to assume that the four functions of equations (1) possess continuous partial derivatives of the fifth order.
} 
$y, \rho, \sigma, \tau$ :

$$
y_{u}=\rho+\beta y, \quad y_{v}=\sigma+\alpha y, \quad y_{u v}=\tau+\alpha \rho+\beta \sigma+(2 \alpha \beta-\lambda) y,
$$

so that equation (44) may be written where

$$
Y=y_{1} y+y_{2} \rho+y_{3} \sigma+y_{4} \tau,
$$

$$
\begin{aligned}
& y_{1}=Y_{1}+\beta Y_{2}+\alpha Y_{3}+(2 \alpha \beta-\lambda) Y_{4}, \\
& y_{2}=Y_{2}+\alpha Y_{4}, \quad y_{3}=Y_{3}+\beta Y_{4}, \quad y_{4}=Y_{4} .
\end{aligned}
$$

If the unit point of the coördinate system be again suitably chosen, the coördinates of the point $Y$ referred to the tetrahedron $y \rho \sigma \tau$ will be the coefficients $y_{1}, y_{2}, y_{3}, y_{4}$ of (46). Using equations (45), we obtain for the values of these coördinates

$$
\begin{aligned}
& y_{1}=1+\beta u-\frac{1}{2}(f+2 b \alpha) u^{2} \\
& +\frac{1}{6}\left[\alpha^{(30)}+\beta \beta^{(30)}+\alpha \gamma^{(30)}+(2 \alpha \beta-\gamma) \delta^{(30)}\right] u^{3}+\cdots, \\
& y_{2}=u+\frac{1}{6}\left(\beta^{(30)}+\alpha \delta^{(30)}\right) u^{3}+\frac{1}{24}\left(\beta^{(40)}+\alpha \delta^{(40)}\right) u^{4}+\cdots \text {, } \\
& y_{3}=-b u^{2}+\frac{1}{6}\left(\gamma^{(30)}+\beta \delta^{(30)}\right) u^{3}+\frac{1}{24}\left(\gamma^{(40)}+\beta \delta^{(40)}\right) u^{4}+\cdots \text {, } \\
& y_{4}=\frac{1}{6} \delta^{(30)} u^{3}+\frac{1}{2} \delta^{(40)} u^{4}+\cdots \text {. }
\end{aligned}
$$

Now let us project the asymptotic curve under consideration upon the tangent plane to $S$ at $y$, i. e., upon the plane $y_{4}=0$, using as center of projection the point $\tau$, i. e., the point $(0,0,0,1)$. Then the point $Y\left(y_{1}, y_{2}\right.$, $\left.y_{3}, y_{4}\right)$ is projected into the point $\eta$, whose coördinates are evidently $\left(y_{1}\right.$, $\left.y_{2}, y_{3}, 0\right)$. Now, it is our purpose to consider the projected configuration in the tangent plane $y_{4}=0$, so we shall find it advantageous to refer this plane to the triangle of reference $y \rho \sigma$, with a suitable unit point. Referred to this triangle the point $Y$ will have coördinates $\left(y_{1}, y_{2}, y_{3}\right)$. The first three of equations (48) are therefore the parametric equations of the projected asymptotic. Let us denote this plane curve by $C_{u}^{\prime}$, recalling that the corresponding asymptotic curve itself is denoted as usual by $C_{u}$.

We proceed now to find the equation of the osculating conic to the projected asymptotic $C_{u}^{\prime}$ at $y$. We must form a homogeneous expression of the second degree in $y_{1}, y_{2}, y_{3}$ such that, if these quantities be replaced by their expansions (48) in powers of $u$, the value of the said homogeneous quadratic in terms of $u$ does not contain any power of $u$ lower than the fifth. It is obvious that we need calculate only $y_{2}^{2}, y_{1} y_{3}, y_{2} y_{3}, y_{3}^{2}$, and form the proper combination of these terms. We have from (48)

$$
\begin{aligned}
y_{2}^{2} & =u^{2}+\frac{1}{3}\left(\beta^{(30)}+\alpha \delta^{(30)}\right) u^{4}+\cdots, \\
y_{1} y_{3} & =-b u^{2}+\frac{1}{6}\left(\gamma^{(30)}+\beta \delta^{(30)}-6 b \beta\right) u^{3}+\{\} u^{4}+\cdots \\
y_{2} y_{3} & =-b u^{3}+\frac{1}{6}\left(\gamma^{(30)}+\beta \delta^{(30)}\right) u^{4}+\cdots \\
y_{3}^{2} & =b^{2} u^{4}+\cdots
\end{aligned}
$$


the coefficient of $u^{4}$ in the expression for $y_{1} y_{3}$ is rather complicated, and of no immediate interest for us. We need now the values of $\gamma^{(30)}$ and $\delta^{(30)}$. Differentiation of the first of equations (4) gives

$$
y_{u u u}=-2 b y_{u v}-f y_{u}-2 b_{u} y_{v}-f_{u} y,
$$

so that $\gamma^{(30)}=-2 b_{u}, \delta^{(30)}=-2 b$. Substituting these only in the second of equations (49), we have

$$
y_{1} y_{3}=-b u^{2}-\frac{1}{3}\left(b_{u}+4 b \beta\right) u^{3}+\{\} u^{4}+\cdots .
$$

From this and the others of equations (49), we obtain at once, without performing any division,

$$
b^{2} y_{2}^{2}+b y_{1} y_{3}-\frac{1}{3}\left(b_{u}+4 b \beta\right) y_{2} y_{3}=Q u^{4}+\cdots,
$$

where $Q$ is a fairly complicated expression, not in general zero, whose actual value is immaterial. This equation and the last of equations (49) show that all of the conics which have contact of the third order with the projected asymptotic $C_{u}^{\prime}$ at $y$ are contained in the pencil

$$
3 b^{2} y_{2}^{2}+3 b y_{1} y_{3}-\left(b_{u}+4 b \beta\right) y_{2} y_{3}+\omega y_{2}^{2}=0,
$$

where $\omega$ is an arbitrary parameter. The osculating conic to $C_{u}^{\prime}$ is contained in this pencil, and its equation is

$$
3 b^{2} y_{2}^{2}+3 b y_{1} y_{3}-\left(b_{u}+4 b \beta\right) y_{2} y_{3}-Q y_{3}^{2}=0 .
$$

Similarly, the asymptotic $C_{v}$ is projected from the point $\tau$ into a curve $C_{v}^{\prime}$ in the tangent plane to $S$ at $y$, and the pencil of conics having contact of the third order with $C_{v}^{\prime}$ at $y$ is

$$
3{a^{\prime}}^{2} y_{3}^{2}+3 a^{\prime} y_{1} y_{2}-\left(a_{0}^{\prime}+4 a^{\prime} \alpha\right) y_{2} y_{3}+\omega^{\prime} y_{2}^{2}=0
$$

where $\omega^{\prime}$ is an arbitrary parameter. The osculating conic is obtained by putting $\omega^{\prime}=Q^{\prime}$, where $Q^{\prime}$ is an expression similar to $Q$.

We shall refer to the conics (50) and (52) respectively as the four-point conics of the projected asymptotics $C_{u}^{\prime}$ and $C_{v}^{\prime}$, and shall denote them by $K_{u}$ and $K_{v}$. But in the subsequent discussion it will be desirable to use continually the fact that the conics lie in the tangent plane of the surface, and we therefore prefer to avoid having to recall this repeatedly, by describing the configuration in terms of the projected asymptotics. The osculating conics of the projected asymptotics are also the osculating conics of the asymptotics themselves.*

12. The Canonical congruences of the first and second kinds. CharACTERIZATION AND GENERALIZATION OF SCROLL DIRECTRIX CONGRUENCES

It is to be noted that in projecting the asymptotic curves upon the tangent plane, we chose as the center of projection an arbitrary point $\tau$ which, when

*Wilczynski, Projective Differential Geometry of Curves and Ruled Surfaces, p. 250. Trans. Am. Matb. Soc. 8 
joined to the point $y$, gave a line $l^{\prime}$ of a congruence $\Gamma^{\prime}$. Now, the entire configuration and analytic work are determined as soon as the point $\tau$ is chosen; in passing, we remark that for a fixed $l^{\prime}$, any point $\tau$ thereon will lead to the same equations for the four-point conics, since equations (50) and (52) are independent of $\lambda$. The conics themselves are of course the same for different choices of $\tau$, in virtue of the fact pointed out at the end of the preceding sections; that the equations of these conics are also unaltered for different values of $\lambda$, in spite of the change in the tetrahedron of reference, follows because the triangle of reference in the tangent plane remains the same. The vertices of the triangle are obviously fixed; the unit point of the system of coördinates is likewise unchanged, because it is really chosen without reference to the point $\tau$.

There are certain lines $l^{\prime}$ which are characterized completely by their geometric relation to the four-point conics which they determine, irrespective of the exact position of the point $\tau$ on the line. We shall in the present section define an important line of this type, for each point of the surface $S$, and thereby define a certain congruence $\Gamma^{\prime}$ and with it the reciprocal congruence $\Gamma$.

Let $\Gamma$ and $\Gamma^{\prime}$ be any pair of reciprocal congruences. The line $l$ of $\Gamma$, i. e., the line $\rho \sigma$, has the equation $y_{1}=0$ in the plane $y \rho \sigma$ referred to the coördinate system of the preceding section. This line meets any four-point conic $K_{u}$ of the pencil (50) in two points determined by the equation

$$
3 b^{2} y_{2}^{2}-\left(b_{u}+4 b \beta\right) y_{2} y_{3}+\omega y_{3}^{2}=0 \text {. }
$$

These two points separate harmonically the points $\rho, \sigma$ if and only if the middle term of the quadratic (53) is absent, i. e., $\beta=-b_{u} / 4 b$.

Let us say that two lines in a plane are reciprocal polars of a conic if either passes through the pole of the other with respect to the conic. Then we may state our result as follows. The asymptotic tangents of the surface $S$ at the point $y$ are reciprocal polars of each of the four-point conics of the projected asymptotic $C_{u}^{\prime}$ if and only if the center of projection which gives rise to these conics is on the line joining $y$ to the point $z=y_{u v}-\alpha y_{u}-\beta y_{v}$, where

$$
\beta=-\frac{1}{4} \frac{b_{u}}{b} \text {. }
$$

This gives us an important covariant point on the asymptotic tangent $y y_{u}$, viz.,

$$
\mathbf{r}=y_{u}+\frac{b_{u}}{4 b} y
$$

Similarly, the asymptotic tangents are reciprocal polars of the four-point conics of the projected asymptotics $C_{v}^{\prime}$ if and only if

$$
\alpha=-\frac{1}{4} \frac{a_{v}^{\prime}}{a^{\prime}} \text {. }
$$


The covariant point

$$
\mathbf{s}=y_{v}+\frac{a_{v}^{\prime}}{4 a^{\prime}} y
$$

is therefore determined on the asymptotic tangent $y y_{v}$.

We are now prepared to characterize geometrically the scroll directrix congruences defined in another way by Sullivan, which we found occasion to mention in $\S 5$. Let the surface $S$ be ruled, but not a quadric, and let the asymptotics $C_{u}(u=$ const. $)$ be its rulings. Then according to equations (20), the scroll directrix of the first kind joins the points

where

$$
\rho=y_{u}-\beta y, \quad \sigma=y_{v}-\alpha y,
$$

$$
\alpha=-\frac{a_{n}^{\prime}}{4 a^{\prime}}, \quad \beta=\frac{a_{u}^{\prime}}{2 a^{\prime}} .
$$

The value of $\alpha$ is that given by (56), so that, combining the results of the present section with those of $\S 5$, we may state the following geometric characterization of the scroll directrix congruences.

The scroll directrix congruences of the first and second kinds for a ruled surface $S$ are completely characterized by the following properties:

1. They are reciprocal congruences.

2. Their developables correspond to the same net of curves on the surface $S$.

3. The asymptotic tangents of $S$ are reciprocal polars of the four-point conics of the projected curved asymptotics.

The formulas for the scroll directrices do not break down for the case in which the surface $S$ is not ruled, but define instead a new pair of covariant lines which we may characterize without difficulty. Let us assume, then, that neither $a^{\prime}$ nor $b$ is zero, and that

$$
\alpha=-\frac{a_{v}^{\prime}}{4 a^{\prime}}, \quad \beta=\frac{a_{u}^{\prime}}{2 a^{\prime}} .
$$

The $\Gamma$ curves no longer coincide with the $\Gamma^{\prime}$-curves, but in equations (9) and (11), which define these curves, we now have the last two coefficients of the one coinciding with the last two coefficients of the other. Therefore the sum of the roots of equation (9) is equal to the sum of the roots of equation (11), both equations being regarded as quadratics in $d v / d u$. Let $t_{2}$ denote as usual the asymptotic tangent to $C_{v}(u=$ const. $)$. Then the harmonic conjugate of $t_{2}$ with respect to the $\Gamma$-tangents coincides with the harmonic conjugate of $t_{2}$ with respect to the $\Gamma^{\prime}$-tangents. In other words, the $\Gamma$-tangents and the $\Gamma^{\prime}$-tangents are pairs of the same involution, one of the double elements of which is the asymptotic tangent $t_{2}$. Therefore, the reciprocal congruences $\Gamma$ and $\Gamma^{\prime}$ for which $\alpha=-a_{v}^{\prime} / 4 a^{\prime}, \beta=a_{u}^{\prime} / 2 a^{\prime}$ are completely characterized by the following properties: 
1. Their $\Gamma$-tangents and $\Gamma^{\prime}$-tangents are pairs of the same involution, of which a double element is the tangent to the asymptotic $C_{v}(u=$ const. $)$.

2. The asymptotic tangents are reciprocal polars of the four-point conics of the projected asymptotic $C_{v}(u=$ const. $)$.

More important than any of the congruences just characterized are the pair for which

$$
\alpha=-\frac{a_{v}^{\prime}}{4 a^{\prime}}, \quad \beta=-\frac{b_{u}}{4 b}
$$

There exists one and only one pair of reciprocal congruences, for any non-ruled surface $S$, having the property that the asymptotic tangents are reciprocal polars with respect to all of the four-point conics of the corresponding projected asymptotics. The congruence $\Gamma$ is generated by the line joining the points

$$
\mathbf{r}=y_{u}+\frac{b_{u}}{4 b} y, \quad \mathbf{s}=y_{v}+\frac{a^{\prime}}{4 a^{\prime}} y
$$

and the congruence $\Gamma^{\prime}$ by the line joining the points $y$ and

$$
\mathbf{z}=y_{u v}+\frac{a_{v}^{\prime}}{4 a^{\prime}} y_{u}+\frac{b_{u}}{4 b} y_{v}
$$

We shall find that the lines rs and $y z$ are two edges of a local tetrahedron of reference which gives rise to a very important canonical expression of a point of the surface in non-homogeneous coördinates. It is for this reason that we shall refer to the lines rs and $y z$ defined by equations (58) and (59) as the canonical edges of the first and second kind respectively, and to the congruences generated by these lines as the canonical congruences of the first and second kinds.

An interesting special case arises when the canonical edges which we have just defined and characterized coincide with the directrices. A necessary and sufficient condition for this is that

$$
\frac{b_{u}}{4 b}=-\frac{a_{u}^{\prime}}{2 a^{\prime}}, \quad \frac{a_{v}^{\prime}}{4 a^{\prime}}=-\frac{b_{v}}{2 b}
$$

or in other words that

$$
\left(a^{\prime 2} b\right)_{u}=0, \quad\left(a^{\prime} b^{2}\right)_{v}=0 .
$$

A particular class of surfaces is characterized by these equations, viz., those for which at each point the canonical cubic hyperosculates the asymptotics.* We have therefore obtained a geometric characterization of these surfaces which is expressed entirely by means of simple geometric concepts.

* Cf. E. J. Wilczynski, On a certain class of self-projective surfaces. These T r a n s a c t i o n s, vol. 14 (1913), pp. 421-443. 
13. Darboux's Canonical expansion in NON-HOMOgENEOUS CoÖRdinates

Let $y$ be a fixed point of the surface; without any loss of generality we may suppose it to have the curvilinear coördinates $(0,0)$. Then any point of the surface in the vicinity of $y$ is given by the Taylor series

$$
Y=y+u y_{u}+v y_{v}+\frac{1}{2}\left(u^{2} y_{u u}+2 u v y_{u v}+v^{2} y_{v v}\right)+\cdots .
$$

In accordance with equation (43), all the derivatives of $y$ may be expressed uniquely as linear combinations of $y, y_{u}, y_{v}, y_{u v}$, so that we may write

$$
Y=Y_{1} y+Y_{2} y_{u}+Y_{3} y_{v}+Y_{4} y_{u v},
$$

where

$$
\begin{aligned}
Y_{1}= & 1-\frac{1}{2} f u^{2}-\frac{1}{2} g v^{2}+\cdots, \\
Y_{2}= & u-a^{\prime} v^{2}+\frac{1}{6}\left(\beta^{(30)} u^{3}+3 \beta^{(21)} u^{2} v+3 \beta^{(12)} u v^{2}+\beta^{(03)} v^{3}\right)+\cdots, \\
Y_{3}= & v-b u^{2}+\frac{1}{6}\left(\gamma^{(30)} u^{3}+3 \gamma^{(21)} u^{2} v+3 \gamma^{(12)} u v^{2}+\gamma^{(03)} v^{3}\right)+\cdots, \\
Y_{4}= & u v+\frac{1}{6}\left(\delta^{(30)} u^{3}+3 \delta^{(21)} u^{2} v+3 \delta^{(12)} u v^{2}+\delta^{(03)} v^{3}\right) \\
& +\frac{1}{2} \frac{1}{4}\left(\delta^{(40)} u^{4}+4 \delta^{(31)} u^{3} v+6 \delta^{(22)} u^{2} v^{2}+4 \delta^{(13)} u v^{3}+\delta^{(04}\right)+\cdots
\end{aligned}
$$

The quantities $Y_{1}, Y_{2}, Y_{3}, Y_{4}$ are obviously the coördinates of $Y$ referred to the tetrahedron with vertices at the points $y, y_{u}, y_{v}, y_{u v}$, and with a suitable unit point.

We shall now choose as three vertices of a new tetrahedron of reference the points $y, \rho=y_{u}-\beta y, \sigma=y_{v}-\alpha y$, and as the fourth vertex the point

$$
\tau=y_{u v}-\lambda y_{u}-\mu y_{v}-\nu y,
$$

where $\lambda, \mu, \nu$ have as yet no relation to $\alpha, \beta$. In fact, the vertices $\rho, \sigma, \tau$ are quite arbitrary, except that $\rho$ and $\sigma$ lie on the asymptotic tangents of the point $y$. If in (61) we replace $y_{u}, y_{v}, y_{u v}$ by their values in terms of $y, \rho$, $\sigma, \tau$, we obtain the equation

$$
Y=y_{1} y+y_{2} \rho+y_{3} \sigma+y_{4} \tau,
$$

where

$$
\begin{gathered}
y_{1}=Y_{1}+\beta Y_{2}+\alpha Y_{3}+(\nu+\beta \lambda+\alpha \mu) Y_{4}, \\
y_{2}=Y_{2}+\lambda Y_{4}, \quad y_{3}=Y_{3}+\mu Y_{4}, \quad y_{4}=Y_{4} .
\end{gathered}
$$

Referred to the new tetrahedron of reference, the point $Y$ has the coördinates $y_{1}, y_{2}, y_{3}, y_{4}$, if the unit point be properly chosen. The expressions (56) may be calculated by means of (62), and are found to be

$$
\begin{aligned}
\begin{aligned}
& y_{1}=1+\beta u+\alpha v-\frac{1}{2}(f+2 b \alpha) u^{2}+(\nu+\beta \lambda+\alpha \mu) u v \\
&-\frac{1}{2}\left(g+2 a^{\prime} \beta\right) v^{2}+\cdots, \\
& y_{2}=u+\lambda u v-a^{\prime} v^{2}+\frac{1}{6}\left[\left(\beta^{(30)}+\lambda \delta^{(30)}\right) u^{3}+3\left(\beta^{(21)}+\lambda \delta^{(21)}\right) u^{2} v\right.
\end{aligned}
\end{aligned}
$$




$$
\begin{array}{r}
\left.+3\left(\beta^{(12)}+\lambda \beta^{(12)}\right) u v^{2}+\left(\beta^{(03)}+\lambda \delta^{(03)}\right) v^{3}\right]+\cdots, \\
y_{3}=v-b u^{2}+\mu u v+\frac{1}{6}\left[\left(\gamma^{(30)}+\mu \delta^{(30)}\right) u^{3}+3\left(\gamma^{(21)}+\mu \delta^{(21)}\right) u^{2} v\right. \\
\left.+3\left(\gamma^{(12)}+\mu \delta^{(12)}\right) u v^{2}+\left(\gamma^{(03)}+\mu \delta^{(03)}\right) v^{3}\right]+\cdots, \\
y_{4}=u v+\frac{1}{6}\left(\delta^{(30)} u^{3}+3 \delta^{(21)} u^{2} v+3 \delta^{(12)} u v^{2}+\delta^{(03)} v^{3}\right) \\
+\frac{1}{24}\left(\delta^{(40)} u^{4}+4 \delta^{(31)} u^{3} v+6 \delta^{(22)} u^{2} v^{2}+4 \delta^{(13)} u v^{3}+\delta^{(04)} v^{4}\right)+\cdots,
\end{array}
$$

where in each case the terms omitted are of higher order than those last written.

We shall now introduce non-homogeneous coördinates by putting

$$
\xi=\frac{y_{2}}{y_{1}}, \quad \eta=\frac{y_{3}}{y_{1}}, \quad \zeta=\frac{y_{4}}{y_{1}} .
$$

After obtaining the power series for $\xi, \eta, \zeta$, we may eliminate the parameters $u, v$, and express $\zeta$ as a power series in $\xi, \eta$. We shall find it convenient first to calculate this expansion only through the terms of the third order, and then make use of the simplifications introduced to calculate the terms of the fourth order. We have

so that

$$
\frac{1}{y_{1}}=1-\beta \mu-\alpha v+\cdots,
$$

$$
\begin{aligned}
& \xi=u-\beta u^{2}+(\lambda-\alpha) u v-a^{\prime} v^{2}+\cdots \\
& \eta=v-b u^{2}+(\mu-\beta) u v-\alpha v^{2}+\cdots \\
& \zeta=u v+\frac{1}{6}\left[\delta^{(30)} \mu^{3}+3\left(\delta^{(21)}-2 \beta\right) u^{2} v\right. \\
& \left.\quad+3\left(\delta^{(12)}-2 \alpha\right) u v^{2}+\delta^{(03)} v^{3}\right]+\cdots
\end{aligned}
$$

From the first two of these we find that

$$
\begin{gathered}
\xi \eta=u v-b u^{3}+(\mu-2 \beta) u^{2} v+(\lambda-2 \alpha) u v^{2}-a^{\prime} v^{3}+\cdots, \\
\xi^{3}=u^{3}+\cdots, \quad \xi^{2} \eta=u^{2} v+\cdots, \quad \xi \eta^{2}=u v^{2}+\cdots, \quad \eta^{3}=v^{3}+\cdots,
\end{gathered}
$$

each expansion being correct to terms of the third crder inclusive. We may find the values of $\delta^{(30)}, \delta^{(21)}, \delta^{(12)}, \delta^{(03)}$ by differentiating equations (4), and obtain, in fact,

$$
\delta^{(30)}=-2 b, \quad \delta^{(21)}=0, \quad \delta^{(12)}=0, \quad \delta^{(03)}=-2 a^{\prime},
$$

so that we finally have, correct to terms of the third order inclusive,

$$
\zeta=\xi \eta+\frac{2}{3} b \xi^{3}-(\mu-\beta) \cdot \xi^{2} \eta-(\lambda-\alpha) \xi \eta^{2}+\frac{2}{3} a^{\prime} \eta^{3}+\cdots .
$$

We may remove the terms in $\xi^{2} \eta$ and $\xi \eta^{2}$ by cheosing $\mu=\beta, \lambda=\alpha$, or in 
other words by making the line $y \tau$ the reciprocal of the line $\rho \sigma$. The development then becomes

$$
\zeta=\xi \eta+\frac{2}{3} b \xi^{3}+\frac{2}{3} a^{\prime} \eta^{3}+\cdots,
$$

and it remains for us to calculate the terms of the fourth order. To terms of the fourth order we have, of course,

$$
\begin{gathered}
u^{4}=\xi^{4}+\cdots, \quad u^{3} v=\xi^{3} \eta+\cdots, \quad u^{2} v^{2}=\xi^{2} \eta^{2}+\cdots, \\
u v^{3}=\xi \eta^{3}+\cdots, \quad v^{4}=\eta^{4}+\cdots,
\end{gathered}
$$

so that we need calculate only the fourth-order terms in the expression

$$
F(\xi, \eta, \zeta) \equiv \zeta-\xi \eta-\frac{2}{3} b \xi^{3}-\frac{2}{3} a^{\prime} \eta^{3}
$$

We have from the first of equations (66)

$\frac{1}{y_{1}}=1-\beta u-\alpha v+\frac{1}{2}\left(f+2 b \alpha+2 \beta^{2}\right) u^{2}-\nu u v+\frac{1}{2}\left(g+2 a^{\prime} \beta+2 \alpha^{2}\right) v^{2}+\cdots$,

in which we have replaced $\lambda, \mu$ by $\alpha, \beta$. Making the same substitution in the others of equations (66) and using equations (69) as well as the equations

$$
\begin{gathered}
\beta^{(30)}=-f, \quad \beta^{(21)}=4 a^{\prime} b, \quad \beta^{(12)}=-\left(g+2 a_{u}^{\prime}\right), \quad \beta^{(03)}=-2 a_{v}^{\prime}, \\
\gamma^{(30)}=-2 b_{u}, \quad \gamma^{(21)}=-\left(f+2 b_{v}\right), \quad \gamma^{(12)}=4 a^{\prime} b, \quad \gamma^{(03)}=-g, \\
\delta^{(40)}=-4 b_{u}, \quad \delta^{(31)}=-\left(f+2 b_{v}\right), \quad \delta^{(22)}=4 a^{\prime} b, \\
\delta^{(13)}=-\left(g+2 a_{u}^{\prime}\right), \quad \delta^{(04)}=-4 a_{v}^{\prime},
\end{gathered}
$$

which may be obtained without difficulty by differentiation of equations (4), we find after a straightforward calculation that

$$
\begin{aligned}
& \xi=u-\beta u^{2}-a^{\prime} v^{2}+\frac{1}{3}\left(f+2 b \alpha+3 \beta^{2}\right) u^{3}+\left(2 a^{\prime} b-\alpha \beta-\nu\right) u^{2} v \\
& +\left(2 a^{\prime} \beta-a_{u}^{\prime}\right) u v^{2}+\frac{1}{3}\left(2 a^{\prime} \alpha-a_{v}^{\prime}\right) v^{3}+\cdots, \\
& \eta=v-b u^{2}-\alpha v^{2}+\frac{1}{3}\left(2 b \beta-b_{u}\right) u^{3}+\left(2 b \alpha-b_{v}\right) u^{2} v \\
& +\left(2 a^{\prime} b-\alpha \beta-\nu\right) u v^{2}+\frac{1}{3}\left(g+2 a^{\prime} \beta+3 \alpha^{2}\right) v^{3}+\cdots, \\
& \zeta=u v-\frac{1}{3}\left(b u^{3}+3 \beta u^{2} v+3 \alpha u v^{2}+a^{\prime} v^{3}\right)+\frac{1}{5}\left(2 b \beta-b_{u}\right) u^{4} \\
& +\frac{1}{3}\left(f-b_{v}+3 \beta^{2}+4 b \alpha\right) u^{3} v+\left(a^{\prime} b-\nu\right) u^{2} v^{2} \\
& +\frac{1}{3}\left(g-a_{u}^{\prime}+3 \alpha^{2}+4 a^{\prime} \beta\right) u v^{3}+\frac{1}{6}\left(2 a^{\prime} \alpha-a_{0}^{\prime}\right) v^{4}+\cdots .
\end{aligned}
$$

From these we find that, to terms of the fourth order,

$$
\begin{aligned}
& \xi \eta=u v-b u^{3}- \beta u^{2} v-\alpha u v^{2}-a^{\prime} v^{3}+\frac{1}{3}\left(5 b \beta-b_{u}\right) u_{4} \\
&+\frac{1}{3}\left(f-3 b_{v}+3 \beta^{2}+8 b \alpha\right) u^{3} v+\left(5 a^{\prime} b-\alpha \beta-2 v\right) u^{2} v^{2} \\
&+\frac{1}{3}\left(g-3 a_{u}^{\prime}+3 \alpha^{2}+8 a^{\prime} \beta\right) u v^{3}+\frac{1}{3}\left(5 a^{\prime} \alpha-a_{v}\right) v^{4}+\cdots, \\
& \xi^{3}=u^{3}-3 \beta u^{4}-3 a^{\prime} u^{2} v^{2}+\cdots, \quad \eta^{3}=v^{3}-3 b u^{2} v^{2}-3 \alpha v^{4}+\cdots
\end{aligned}
$$


The expression (71) may now be calculated, and is easily found to be

$$
\begin{aligned}
F(\xi, \eta, \zeta)=\frac{1}{6}\left(4 b \beta+b_{u}\right) u^{4} & +\frac{2}{3}\left(b_{v}-2 b \alpha\right) u^{3} v+(\nu+\alpha \beta) u^{2} v^{2} \\
& +\frac{2}{3}\left(a_{u}^{\prime}-2 a^{\prime} \beta\right) u v^{3}+\frac{1}{6}\left(4 a^{\prime} \alpha+a_{v}^{\prime}\right) v^{4}+\cdots
\end{aligned}
$$

Therefore, to terms of the fourth order inclusive,

$$
\begin{aligned}
\zeta= & \xi \eta+\frac{2}{3} b \xi^{3}+\frac{2}{3} a^{\prime} \eta^{3}+\frac{1}{6}\left(4 b \beta+b_{u}\right) \xi^{4}+\frac{2}{3}\left(b_{v}-2 b \alpha\right) \xi^{3} \eta \\
& +(\nu+\alpha \beta) \xi^{2} \eta^{2}+\frac{2}{3}\left(a_{u}^{\prime}-2 a^{\prime} \beta\right) \xi \eta^{3}+\frac{1}{6}\left(4 a^{\prime} \alpha+a_{v}^{\prime}\right) \eta^{4}+\cdots
\end{aligned}
$$

Let us assume that at the point about which the above development is made neither $a^{\prime}$ nor $b$ is zero. The surface in the neighborhood of the point is then not ruled, and we may in this case choose $\alpha, \beta$, and $\nu$ in such a way as to make the coefficients of $\xi^{4}, \xi^{2} \eta^{2}$, and $\eta^{4}$ in the development vanish. The required values of $\alpha, \beta, \nu$ are

$$
\alpha=-\frac{c_{v}^{\prime}}{4 a^{\prime}}, \quad \beta=-\frac{b_{u}}{4 b}, \quad \nu=-\alpha \beta,
$$

and the vertices of the tetrahedron of reference are fixed thereby. The development is now

$$
\zeta=\xi \eta+\frac{2}{3} b \xi^{3}+\frac{2}{3} a^{\prime} \eta^{3}+\frac{1}{3}\left(2 b_{v}+b \frac{a_{v}^{\prime}}{a^{\prime}}\right) \xi^{3} \eta+\frac{1}{3}\left(2 a_{u}^{\prime}+a^{\prime} \frac{b_{u}}{b}\right) \xi \eta^{3}+\cdots
$$

we proceed to make a final simplification thereof. If we introduce new variables $x, y, z$, related to the old by the equations

$$
\xi=\phi x, \quad \eta=\psi y, \quad \zeta=\omega z,
$$

the first part of (76) becomes

$$
z=\frac{\phi \psi}{\omega} x y+\frac{2}{3} \frac{\phi^{3}}{\omega} b x^{3}+\frac{2}{3} \frac{\psi^{3}}{\omega} a^{\prime} y^{3}+\cdots
$$

We shall now choose $\phi, \psi, \omega$ so that

i. e.,

$$
\phi \psi=\omega, \quad 4 b \phi^{3}=\omega, \quad 4 a^{\prime} \psi^{3}=\omega,
$$

so that

$$
4 b \phi^{2}=\psi, \quad 4 a^{\prime} \psi^{2}=\phi,
$$

where

$$
1=, \quad \psi=\frac{1}{4 \sqrt[3]{B}},
$$

Making the final substitutions, we may state the result:

At a regular point of a non-ruled surface, the surface may be represented by the following canonical development in non-homogeneous coördinates:

$$
z=x y+\frac{1}{6}\left(x^{3}+y^{3}\right)+\frac{1}{2} 4\left(I^{\prime} x^{3} y+J^{\prime} x y^{3}\right)+\cdots,
$$


where

$$
I^{\prime}=\frac{A_{v}}{2 A \sqrt[3]{B}}, \quad J^{\prime}=\frac{B_{u}}{2 B \sqrt[3]{A}}
$$

and all other coefficients are absolute invariants.

- This canonical development is essentially one which was first obtained by Darboux, ${ }^{*}$ who arrived at his expansion by a procedure which was entirely analytic. He made no attempt to describe geometrically the tetrahedron of reference which gives rise to the development; the results of $\S 12$ will enable us to supply this geometric characterization. In fact, two of the edges of the canonical tetrahedron, as we may call it, are the lines which we called the canonical edges of the point $y$ in $\S 12$. The point $y$ is one vertex of the canonical tetrahedron, two others are furnished by the points

$$
\mathbf{r}=y_{u}+\frac{b_{u}}{4 b} y, \quad \mathbf{s}=y_{v}+\frac{a_{v}^{\prime}}{4 a^{\prime}} y
$$

in which the canonical edge of the first kind meets the asymptotic tangents of the point $y$, and the fourth vertex is the point

$$
\mathbf{t}=y_{u v}+\frac{a_{v}^{\prime}}{4 a^{\prime}} y_{u}+\frac{b_{u}}{4 b} y_{v}+\frac{a_{v}^{\prime} b_{u}}{16 a^{\prime} b} y
$$

It remains, then, to characterize this fourth point geometrically, as well as to describe our choice of the unit-point of the coördinate system which gives rise to the canonical development. Wilczynski has defined geometrically what he calls the canonical quadric of a point $y$ of the surface. Referred to the tetrahedron $y y_{u} y_{v} y_{u v}$, the equation of this quadric is $\dagger$

$$
x_{1} x_{4}-x_{2} x_{3}=0 \text {. }
$$

Let us find the point of intersection of the line joining the points $y$ and

$$
z=y_{u v}-\alpha y_{u}-\beta y_{v}
$$

with the canonical quadric. Any point on this line is given by an expression of the form

$$
\tau=y_{u v}-\alpha y_{u}-\beta y_{v}-\nu y,
$$

where we wish to determine $\nu$ so that the point $\tau$ shall lie on the quadric (83). Referred to the tetrahedron $y y_{u} y_{v} y_{u v}$, the point $\tau$ has coördinates

*Bulletin des Sciences Mathematiques, series 2, vol. 4 (1880), pp. 348-384. Darboux's development is of the form $z=x y+x^{3}+y^{3}+a x^{3} y+b x y^{3}+\cdots$, and may be transformed into ours by means of a substitution of the form (77).

$\dagger$ We have already met with this quadric, at the end of $\S 7$. It was with the present discussion in mind that we there pointed out the desirability of characterizing this quadric by a method independent of that used by Wilczynski, because of numerous simplifications which would be introduced thereby both in his work and our own. 
$(-\nu,-\alpha,-\beta, 1)$, so that, substituting these in equation (83), we find that $\nu=-\alpha \beta$, which is the third of equations (75). The fourth vertex of the canonical tetrahedron is therefore the point, distinct from $y$, in which the canonical quadric is met by the canonical edge of the second kind.

We may therefore describe the canonical tetrahedron in the following interesting way: the vertices of the canonical tetrahedron are the four points in which the canonical quadric is met by the canonical edges of the first and second kinds.

In addition to the development (80), there exist other canonical expansions, the most important of which is probably the following, which was also established by Darboux.

$$
z=x y+\frac{1}{6}\left(x^{3}+y^{3}\right)+\frac{1}{24}\left(I x^{4}+J y^{4}\right)+\cdots,
$$

where $I, J$, and all further coefficients are likewise absolute projective invariants. It remained for Wilczynski, however, in his second memoir on curved surfaces, already cited, to furnish a complete characterization of the tetrahedron which gives rise to the expansion (84). We may state this characterization in a form similar to that given for the canonical development (80), as follows. The tetrahedron of reference which gives rise to the development (84) has for vertices the four points in which the canonical quadric is met by the directrices of the first and second kinds.

The four invariants $I, J, I^{\prime}, J^{\prime}$ appearing in the developments (80) and (84) are connected by the equations

$$
I=\frac{1}{2} J^{\prime}, \quad J=\frac{1}{2} I^{\prime} .
$$

Two other canonical expansions exist, one of which arises when the terms in $\xi \eta^{3}$ and $\eta^{4}$ are caused to disappear in (74), besides the term in $\xi^{2} \eta^{2}$; the other is obtained when the terms in $\xi^{4}, \xi^{3} \eta$, and $\xi^{2} \eta^{2}$ are made to vanish. In the first of these cases, we must choose $\alpha=-a_{v}^{\prime} / 4 a^{\prime}, \beta=a_{u}^{\prime} / 2 a^{\prime}$, which are the same as equations (20). The lines $l$ and $l^{\prime}$ which form two edges of the tetrahedron of reference are therefore the generalizations of the scroll directrices of the first and second kinds which we characterized in $\S 12$. The vertices of the tetrahedron are the points where these lines $l$ and $l^{\prime}$ cut the canonical quadric, and if new variables $x, y, z$ are introduced in accordance with equations (77) and (78), the expansion becomes

$$
z=x y+\frac{1}{6}\left(x^{3}+y^{3}\right)+\frac{1}{24}\left(I x^{4}+I^{\prime} x^{3} y\right)+\cdots .
$$

In a similar way may be obtained the expansion

$$
z=x y+\frac{1}{6}\left(x^{3}+y^{3}\right)+\frac{1}{24}\left(J^{\prime} x y^{3}+J y^{4}\right)+\cdots .
$$

In both of these, the invariants $I, I^{\prime}, J, J^{\prime}$ are those defined by equations 
(81) and (85), and all further coefficients are likewise invariants. We have throughout assumed, as before, that neither $a^{\prime}$ nor $b$ is zero. If $b$, for instance, is zero, none of our expansions will be valid; a glance at equation (74) will show, however, that in this case the term in $\xi^{3}$ disappears, and the only simple way to obtain a canonical expansion is to choose $\alpha=-a_{v}^{\prime} / 4 a^{\prime}, \beta=a_{u}^{\prime} / 2 a^{\prime}$, $\nu=-\alpha \beta$, which yields a development further reducible to

$$
z=x y+y^{3}+m x^{2} y^{3}+n x y^{4}+y^{5}+\cdots .
$$

This expansion was obtained by Darboux and Wilczynski,* and (86) is obviously a generalization thereof. The vertices of the tetrahedron of reference are the points in which the osculating quadric is cut by the scroll directrices.

We have observed that in all of the above canonical expansions the vertices of the local tetrahedron of reference are the intersections of the canonical quadric with a pair of reciprocal lines-which are, by the way, reciprocal polars with respect to this quadric. It may not be without interest, therefore, to point out how this choice of vertices causes terms to disappear from the expansion in non-homogeneous coördinates. If an arbitrary tetrahedron of reference be chosen at the outset, the expansion will contain all terms, including a constant term. This constant term disappears if the point $y$ on the surface is one of the vertices; the terms in $\xi$ and $\eta$ vanish if the tangent plane at $y$ is a face of the tetrahedron; the terms in $\xi^{2}$ and $\eta^{2}$ vanish if the asymptotic tangents are edges of the tetrahedron; the terms in $\xi^{2} \eta$ and $\xi \eta^{2}$ disappear if, in addition to the preceding, two edges of the tetrahedron are a pair of reciprocal lines $l$ and $l^{\prime}$; and finally, the term in $\xi^{2} \eta^{2}$ vanishes if the fourth vertex of the tetrahedron is then determined as the second intersection of the line $l^{\prime}$ with the canonical quadric. The tetrahedron is chosen in this way for all of the canonical developments of the present section; the particular pair of reciprocal lines which are chosen as edges of the tetrahedron will finally determine the nature of the rest of the expansion.

Equations (85) show that a necessary condition that the two developments (80) and (84) coincide is that $I$ and $J$ vanish both. But this condition is also sufficient, because it is equivalent to equations (60), which we found in the last section to be a necessary and sufficient condition that the directrices of the first and second kinds coincide respectively with the canonical edges of the first and second kinds. Of course the mere coincidence of the two tetrahedrons would not in itself carry with it the coincidence of the corresponding developments, because what we have said thus far will not ensure also the coincidence of the unit points of the two coördinate systems. An examination of the procedure by which the two developments are obtained will, however, show that the coördinate systems do in fact coincide throughout. In the

\footnotetext{
* Darboux, loc. cit., and Wilczynski, Curved Surfaces, third memoir, these Tran s a c t i o n s, vol. 9 (1908), p. 299.
} 
next section we shall, moreover, determine the geometric character of the unit point of the coördinate system which gives rise to the development (80), and shall, incidentally, show that the same characterization will be applicable, mutatis mutandis, in the case of the expansion (84), and also of (86) and (87).

If $I$ and $J$ are both zero, all four of the developments (80), (84), (86), and (87) will coincide with each other; the vanishing of $I$ alone, however, will be sufficient to ensure the coincidence of (84) and (87), and the vanishing of $J$ alone will ensure the coincidence of (84) and (86). We are therefore in position to furnish a criterion for the vanishing of $I$ alone, or of $J$ alone, expressible entirely in geometric language.

\section{The COMPleted CharaCterization of the CANONICAL TETRAhedRoN}

In his characterization of the coördinate system which gives rise to the development (84), Wilczynski makes use of the cubic surface whose equation,

$$
z=x y+\frac{1}{6}\left(x^{3}+y^{3}\right),
$$

is formed by breaking off the development (84) from the terms of the third order. In terms of this canonical cubic, as he calls it, he defines the canonical quadric, which we have already used in determining the vertex $t$. We may employ this quadric also in characterizing the cubic surface whose equation is (89), but referred to the canonical tetrahedron which gives rise to the development (80).

Referred to the tetrahedron $y y_{u} y_{v} y_{u v}$, the equation of the canonical quadric is

$$
x_{1} x_{4}-x_{2} x_{3}=0 .
$$

We wish to obtain its equation with respect to our canonical tetrahedron. Let $X_{1}, X_{2}, X_{3}, X_{4}$ be the current coördinates (homogeneous) of a point with respect to this tetrahedron, and, as usual, let $x_{1}, x_{2}, x_{3}, x_{4}$ be the coördinates of the same point with respect to the tetrahedron $y y_{u} y_{v} y_{u v}$. We must first find the equations of transformation between the $X_{k}$ 's and the $x_{k}$ 's. We have

$$
x=\frac{X_{2}}{X_{1}}, \quad y=\frac{X_{3}}{X_{1}}, \quad z=\frac{X_{4}}{X_{1}},
$$

so that, by (77), (67), and (65),*

$$
\begin{aligned}
& \phi \frac{X_{2}}{X_{1}}=\frac{x_{2}+\alpha x_{4}}{x_{1}+\beta x_{2}+\alpha x_{3}+\alpha \beta x_{4}}, \\
& \psi \frac{X_{3}}{X_{1}}=\frac{x_{3}+\beta x_{4}}{x_{1}+\beta x_{2}+\alpha x_{3}+\alpha \beta x_{4}}, \\
& \omega \frac{X_{4}}{X_{1}}=\frac{x_{4}}{x_{1}+\beta x_{2}+\alpha x_{3}+\alpha \beta x_{4}} .
\end{aligned}
$$

\footnotetext{
* In equations (65), $Y_{k}$ is to be replaced by $x_{k}, \lambda$ by $\alpha, \mu$ by $\beta$, and $\nu$ by $-\alpha \beta$.
} 
If we denote by $\kappa$ a factor of proportionality, we may therefore write

$$
\begin{gathered}
\kappa X_{1}=x_{1}+\beta x_{2}+\alpha x_{3}+\alpha \beta x_{4}, \\
\kappa \phi X_{2}=x_{2}+\alpha x_{4}, \quad \kappa \psi X_{3}=x_{3}+\beta x_{4}, \quad \kappa \omega X_{4}=x_{4} .
\end{gathered}
$$

Solving these for $x_{1}, x_{2}, x_{3}, x_{4}$, we have

$$
\begin{gathered}
x_{1}=\kappa\left(X_{1}-\beta \phi X_{2}-\alpha \psi X_{3}+\alpha \beta \omega X_{4}\right), \\
x_{2}=\kappa\left(\phi X_{2}-\alpha \omega X_{4}\right), \quad x_{3}=\kappa\left(\psi X_{3}-\beta \omega X_{4}\right), \quad x_{4}=\kappa \omega X_{4} .
\end{gathered}
$$

Therefore, in canonical homogeneous coördinates, the equation of the canonical quadric is

$$
X_{1} X_{4}-X_{2} X_{3}=0 \text {. }
$$

We proceed now to characterize geometrically the cubic surface whose equation in non-homogeneous canonical coördinates is (89), and in homogeneous canonical coördinates is

$$
X_{1}^{2} X_{4}=X_{1} X_{2} \dot{X}_{3}+\frac{1}{6}\left(X_{2}^{3}+X_{3}^{3}\right) \text {. }
$$

It is not difficult to verify that the most general cubic surface which has a unode at the point $(0,0,0,1)$, and the plane $X_{1}=0$ as its uniplane, must have an equation of the form*

$$
X_{1}^{2} X_{4}=\Phi\left(X_{1}, X_{2}, X_{3}\right),
$$

or, in non-homogeneous coördinates,

$$
z=\Phi(1, x, y)
$$

where $\Phi$ is a homogeneous cubic in the three arguments indicated. In order that this surface may have contact of the third order with the surface $S$, the canonical expansion (74) must agree with the right-hand member of (87) through the terms of the third order; in other words, the function $\Phi(1, x, y)$ is the right-hand member of equation (89). Therefore, the canonical cubic

$$
X_{1}^{2} X_{4}=X_{1} X_{2} X_{3}+\frac{1}{6}\left(X_{2}^{3}+X_{3}^{3}\right)
$$

is completely characterized by the following properties:

1. It has a unode at the vertex $(0,0,0,1)$ of the canonical tetrahedron of reference.

2. Its uniplane is the face $X_{1}=0$ of this tetrahedron.

3. It has contact of the third order with the surface $S$ at the point $y(1,0,0,0)$.

We are now in position to describe the unit point of the coördinate system which gives rise to the canonical development (80). The points $y, \mathbf{r}, \mathbf{s}, \mathbf{t}$

${ }^{*}$ Cf. Wilczynski, Second memoir on curved surfaces, these Transactions, vol. 9 (1908), pp. 104-105. 
are respectively the points $(1,0,0,0),(0,1,0,0),(0,0,1,0)$, $(0,0,0,1)$ of this system. Let us denote these points by $P_{1}, P_{2}, P_{3}, P_{4}$ respectively. Their geometric relation to the surface $S$ has already been established, as has that of the canonical cubic. Now, the edge $P_{2} P_{3}$ of the tetrahedron of reference has the equations $X_{1}=0, X_{4}=0$, so that it has three points of intersection with the canonical cubic (94) which are given by the equation

$$
X_{2}^{3}+X_{3}^{3}=0 \text {. }
$$

It may be verified without difficulty that these points are the three points of inflection of the cubic curve in which the plane $X_{4}=0$-the tangent plane of $S$-cuts the canonical cubic, but they are already sufficiently characterized geometrically as the points in which the line $P_{2} P_{3}$ meets the canonical cubic. One of these three points is the point $Q(0,1,-1,0)$. The harmonic conjugate of the point $Q$ with respect to $P_{2}$ and $P_{3}$ is obviously the point $Q^{\prime}(0,1,1,0)$.

The plane which passes through the point $Q^{\prime}(0,1,1,0)$ and the edge $P_{1} P_{4}$ of the tetrahedron has the equation $X_{2}=X_{3}$. This plane intersects the canonical cubic in a cubic curve, whose equation in homogeneous coordinates may be obtained by setting $X_{2}=X_{3}$ in equation (94). We may write it

$$
3 X_{1}^{2} X_{4}-3 X_{1} X_{2}^{2}-X_{2}^{3}=0,
$$

and proceed now to determine its points of inflection. The hessian curve of the cubic (96) is readily found to be

$$
X_{1}^{2}\left(X_{1}+X_{2}\right)=0 \text {, }
$$

and its nine intersections with the cubic (96) give the points of inflection of that cubic. The solution $X_{1}=0$ corresponds to a double point, and accounts for six intersections, while $X_{1}+X_{2}=0$ is a line which passes through this double point, where it accounts for two more intersections, and also through the point $M(-3,3,3,-2)$, which is the sole point of inflection of the cubic (96).

The line which passes through the points $P_{4}(0,0,0,1)$ and $M$ $(-3,3,3,-2)$ intersects the face $X_{4}=0$ of the tetrahedron in the point $M^{\prime}(-1,1,1,0)$. This point lies on the line joining the points $P_{1}(1,0,0,0)$ and $Q^{\prime}(0,1,1,0)$, and its harmonic conjugate with respect to these points is the point $M^{\prime \prime}(1,1,1,0)$.

The polar plane of this point $M^{\prime \prime}$ with respect to the canonical quadric (93) is

$$
X_{2}+X_{3}-X_{4}=0 \text {. }
$$

It intersects the line $P_{2} P_{4}$ in the point $M_{1}(0,1,0,1)$, and the line $P_{3} P_{4}$ 
in the point $M_{2}(0,0,1,1)$. The plane of the three points $P_{1}, P_{3}$, and $M_{1}$ has the equation $X_{2}=X_{4}$, the plane of the points $P_{1}, P_{2}$, and $M_{2}$ has the equation $X_{3}=X_{4}$, and the plane of the points $P_{3}, P_{4}$, and $M^{\prime \prime}$ has the equation $X_{1}=X_{2}$. These three planes obviously meet in the point $(1,1,1,1)$, the unit point of the system of canonical coördinates.

In recapitulation, therefore, we may state the

THEOREM. The vertices of the canonical tetrahedron of a regular point $P_{1}$ of a non-ruled surface are the four points in which the canonical edges of the first and second kinds intersect the canonical quadric. Let $P_{2}, P_{3}$ be the points in which the canonical quadric is met by the canonical edge of the first kind, and $P_{1}, P_{4}$ the points in which the canonical edge of the second kind meets this quadric. Let $Q$ be one of the three points in which the edge $P_{2} P_{3}$ intersects the canonicai cubic, and denote by $Q^{\prime}$ the harmonic conjugate of $Q$ with respect to $P_{2}$ and $P_{3}$. The plane through $P_{1}, Q^{\prime}$, and $P_{4}$ intersects the canonical cubic in a cubic curve, which has but one point of inflection, $M$. Let $M^{\prime}$ be the point in which the line $P_{4} M$ meets the plane $P_{1} P_{2} P_{3}$. It lies on the line joining the points $P_{1}$ and $Q^{\prime}$. Let $M^{\prime \prime}$ be the harmonic conjugate of $M^{\prime}$ with respect to $P_{1}$ and $Q^{\prime}$. The polar plane of $M^{\prime \prime}$ with respect to the canonical quadric of the point $P_{1}$ intersects the line $P_{2} P_{4}$ in a point $M_{1}$, and the line $P_{3} P_{4}$ in a point $M_{2}$. The planes $P_{1} P_{3} M_{1}, P_{1} P_{2} M_{2}$, and $P_{3} P_{4} M^{\prime \prime}$ intersect in a point, which is the unit point of the coordinate system which gives rise to the canonical development (80). The presence of the cube root of a rational invariant in the coefficients of this development is explained by the fact that the point $Q$ may be chosen from among three distinct points.

Obviously, the above characterization is applicable without any change whatever-except for easily supplied modifications which arise when the canonical edges are replaced by the directrices, or by the generalized scroll directrices, or by any other pair of reciprocal lines-in fixing the unit point of the coördinate system which gives rise to the development (84), or (86), or (87), or any other canonical development of similar nature.

\section{The CONGRUENCE OF PSEUdo-NORMals}

In establishing projective generalizations of theorems in the metric differential geometry of surfaces, it becomes necessary to provide a substitute for the congruence of normals to the surface. Of course, if it were merely a question of finding a uniquely determined congruence $\Gamma^{\prime}$ of lines protruding from the surface, and projectively defined in terms of the surface, any of the covariant congruences of this description which have appeared in preceding sections would suffice. Wilczynski's directrix congruence of the second kind, or the congruence formed of the canonical edges of the second kind, would be two such congruences. The $\Gamma^{\prime}$-curves of either of these congruences would then take the place of the lines of curvature of the metric theory. 
The congruence of normals, however, has an important property which it would be desirable to retain in any projective generalization. The developables of the congruence meet the surface in its lines of curvature, which form a conjugate net. In other words, using a definition of $\S 8$, the congruence of normals is conjugate to the surface. We must therefore seek not merely a covariant congruence $\Gamma^{\prime}$ projectively determined by the surface, but one which is in addition conjugate to the surface.

That the developables of the congruence of normals meet the surface in a conjugate net is in a certain sense a fortunate accident. A familiar metric property of a developable surface is, that if each of the generators of the developable be turned through the same angle about its point of intersection with an orthogonal trajectory of all the generators, the generators in their new position being likewise orthogonal to this trajectory, then the generators in their new position likewise form a developable. If, therefore, the generators of a developable of the normal congruence of a surface $S$ be turned in this way through a right angle about the curve of intersection of the developable with the surface $S$, the generators in their new positions are tangents to the surface $S$ and cut the said curve of intersection at right angles. They are, moreover, the directions conjugate to the tangents of this curve of intersection, since they form a developable, so that the curve of intersection is a line of curvature on $S$. It is for this reason that the normal congruence is conjugate to the surface; the property follows merely from the general proposition concerning developable surfaces quoted above.

In view of the above considerations, it is indeed remarkable that the congruence generated by the line joining the point $y$ to the point

$$
Z=y_{u v}-\alpha y_{u}-\beta y_{v},
$$

where

$$
\alpha=-\frac{1}{2}\left(\frac{a_{v}^{\prime}}{a^{\prime}}+\frac{b_{v}}{b}\right), \quad \beta=-\frac{1}{2}\left(\frac{a_{u}^{\prime}}{a^{\prime}}+\frac{b_{u}}{b}\right),
$$

is not only conjugate to the surface,-since $\alpha_{u}=\beta_{v}$-but is also covariant to the surface, that is, uniquely determined by the surface in a projective way.

We shall call this congruence the pseudo-normal congruence of the surface, and the line of the congruence which passes through a point $y$ of the surface the pseudo-normal of the point $y$.

It remains to characterize the pseudo-normal of the point $y$ geometrically, and thereby prove it to be a covariant line. The line which is reciprocal to the pseudo-normal lies in the tangent plane to the surface at $y$, and joins the points

$$
R=y_{u}+\frac{1}{2}\left(\frac{a_{u}^{\prime}}{a^{\prime}}+\frac{b_{u}}{b}\right) y, \quad S=y_{v}+\frac{1}{2}\left(\frac{a_{v}^{\prime}}{a^{\prime}}+\frac{b_{v}}{b}\right) y .
$$


We recall also that the asymptotic tangents are met in the points

$$
r=y_{u}-\frac{a_{u}^{\prime}}{2 a^{\prime}} y, \quad s=y_{v}-\frac{b_{v}}{2 b} y
$$

by the directrix of the first kind, and in the points

$$
\mathbf{r}=y_{u}+\frac{b_{u}}{4 b} y, \quad \mathbf{s}=y_{v}+\frac{a_{v}^{\prime}}{4 a^{\prime}} y
$$

by the canonical edge of the first kind. Obviously, the point $\mathbf{r}$ is the harmonic conjugate of $y$ with respect to $R$ and $r$, and $\mathbf{s}$ is the harmonic conjugate of $y$ with respect to $S$ and $s$. The points $R$ and $S$ are therefore determined geometrically, and hence also the pseudo-normal, which is the line reciprocal to $R S$. Obviously, the directrix of the first kind, the canonical edge of the first kind, and the line $R S$ meet in a point in the tangent plane of $y$. We may therefore state the characterization of the pseudo-normal as follows.

The directrix of the first kind and the canonical edge of the first kind corresponding to a point $P$ of the surface meet in a point $P^{\prime}$. The pseudo-normal of the point $P$ is the reciprocal of the line which is the harmonic conjugate of the directrix of the first kind with respect to the canonical edge of the first kind and the line $P P^{\prime}$.

We have thus determined geometrically a covariant congruence $\Gamma^{\prime}$, whose developables cut the surface in a unique conjugate net. This net bears the same relation to the congruence of pseudo-normals as the lines of curvature, which likewise form a conjugate net, do to the congruence of normals to the surface. It is obvious, then, that the congruence of pseudo-normals must play a large part in the projective generalization of metric theorems, and in the characterization of classes of surfaces projectively analogous to important known classes of surfaces. Certain of these generalizations will be indicated presently; we shall first establish some formulas which will be useful in many discussions involving the pseudo-normal congruence or related configurations.

We have thus far been using almost exclusively the differential equations in their canonical form (4). In accordance with the remarks made at the end of $\S 1$, we may write out all of the important formulas if the differential equations are in their unreduced form (2):

$$
\begin{aligned}
y_{u u}+2 a y_{u}+2 b y_{v}+c y & =0, \\
y_{v v}+2 a^{\prime} y_{u}+2 b^{\prime} y_{v}+c^{\prime} y & =0 .
\end{aligned}
$$

Equations (4a) enable us to express at once in terms of the coefficients of these equations any formula which contains only coefficients of equations (4), and does not contain the dependent variable $y$. Let us denote for a moment by $\bar{y}$ the dependent variable in equations (4), and by $y$ the dependent variable in equations (2). Then, according to $\S 1$, the transformation which converts 
system (2) into system (4) is

(3a)

$$
y=\lambda \bar{y}
$$

where

$$
\lambda_{u}=-a \lambda, \quad \lambda_{v}=-b^{\prime} \lambda .
$$

Consequently, the derivatives of $\bar{y}$ must be replaced, in all formulas, by their equivalents in terms of $y$ :

$$
\begin{aligned}
\bar{y}_{u} & =\frac{1}{\lambda}\left(y_{u}+a y\right), \quad \bar{y}_{v}=\frac{1}{\lambda}\left(y_{v}+b^{\prime} y\right), \\
\bar{y}_{u v} & =\frac{1}{\lambda}\left[y_{u v}+b^{\prime} y_{u}+a y_{v}+\left(a_{v}+a b^{\prime}\right) y\right] \\
& =\frac{1}{\lambda}\left[y_{u v}+b^{\prime} y_{u}+a y_{v}+\left(b_{u}^{\prime}+a b^{\prime}\right) y\right] .
\end{aligned}
$$

The two expressions for $y_{u v}$ are identical, since one of the integrability conditions for system (2) is $a_{v}=b_{u}^{\prime}$. In terms of the coefficients and variables of system (2), therefore, the directrix of the first kind is the line which connects the points

$$
r=y_{u}-\left(\frac{a_{u}^{\prime}}{2 a^{\prime}}-a\right) y, \quad s=y_{v}-\left(\frac{b_{v}}{2 b}-b^{\prime}\right) y,
$$

and the directrix of the second kind connects the point $y$ with the point

$$
z=y_{u v}-\left(\frac{b_{v}}{2 b}-b^{\prime}\right) y_{u}-\left(\frac{a_{u}^{\prime}}{2 a^{\prime}}-a\right) y_{v} .
$$

The canonical edge of the first kind connects the points

$$
\mathbf{r}=y_{u}+\left(\frac{b_{u}}{4 b}+a\right) y, \quad \mathbf{s}=y_{v}+\left(\frac{a_{v}^{\prime}}{4 a^{\prime}}+b^{\prime}\right) y,
$$

and the canonical edge of the second kind joins the point $y$ with the point

$$
\mathbf{z}=y_{u v}+\left(\frac{a_{v}^{\prime}}{4 a^{\prime}}+b^{\prime}\right) y_{u}+\left(\frac{b_{u}}{4 b}+a\right) y_{v} .
$$

The line reciprocal to the pseudo-normal connects the points

$$
\begin{aligned}
& R=y_{u}-\frac{1}{2}\left(\frac{a_{u}^{\prime}}{a^{\prime}}+\frac{b_{u}}{b}-2 a\right) y, \\
& S=y_{v}-\frac{1}{2}\left(\frac{a_{v}^{\prime}}{a^{\prime}}+\frac{b_{v}}{b}-2 b^{\prime}\right) y,
\end{aligned}
$$

and the pseudo-normal connects the point $y$ with the point

$$
Z=y_{u v}-\frac{1}{2}\left(\frac{a_{v}^{\prime}}{a^{\prime}}+\frac{b_{v}}{b}-2 b^{\prime}\right) y_{u}-\frac{1}{2}\left(\frac{a_{u}^{\prime}}{a^{\prime}}+\frac{b_{u}}{b}-2 a\right) y_{v} .
$$


We have, as usual, discarded the factor $1 / \lambda$ on account of the homogeneity of the coördinates.

The formulas which have just been derived are useful only when the surface is referred to its asymptotic net. Very often, however, the parametric net is not asymptotic, and in such a case the determination of the asymptotic net, and consequently also of the system of differential equations (2), requires the integration of a quadratic partial differential equation of the first order. We have shown elsewhere, ${ }^{*}$ however, that although the determination of the asymptotic curves may require an integration which it is impossible to carry out explicitly, all the formulas which we have developed thus far in the present paper may be expressed explicitly, and entirely, in terms of the given parametric net. That is, if the parametric net is not conjugate, for instance, the surface may be defined as an integral surface of a completely integrable system of partial differential equations of the form

$$
\begin{aligned}
& y_{u u}=a y_{u v}+b y_{u}+c y_{v}+d y, \\
& y_{v v}=a^{\prime} y_{u v}+b^{\prime} y_{u}+c^{\prime} y_{v}+d^{\prime} y,
\end{aligned}
$$

-where the coefficients have not the significance of the same letters which appear in systems (2) and (4) -and this system may be reduced to one of the form (2), in which the coefficients of $y_{u v}$ are zero, by the integration of the differential equation which defines the asymptotic curves of the surface; nevertheless, the formulas of the present paper, which we have hitherto written in terms of the coefficients and variables appearing in system (2), may be expressed explicitly in terms of the coefficients and variables of system (106), in spite of the fact that the coefficients and variables themselves in equations (106) are not expressible explicitly in terms of those in equations (2). We have, in the paper last cited, established all of the formulas which are necessary for the conversion of formulas expressed in terms of system (2) into the corresponding formulas for system (106). The calculations involved are long, and we shall-leave them for another occasion.

Perhaps the most desirable of all the formulas in terms of a non-asymptotic parametric net would be those which express the condition that the given parametric net be the $\Gamma^{\prime}$-curves of the pseudo-normal congruence, or, as we may call them, the pseudo-normal curves. In this case the parametric net must be conjugate; a starting-point for the necessary calculations; which again are rather tedious, is to be found in a paper by the present writert.

* G. M. Green, On the theory of curved surfaces, and canonical systems in projective differential geometry, these Trans a c t i o s, vol. 16 (1915), pp. 1-12.

$\dagger$ G. M. Green, Projective differential geometry of one-parameter families of space curves, and conjugate nets on a curved surface, American Journal of $\mathrm{Mathematics,} \mathrm{vol.}$ 37 (1915), pp. 215-246. 
16. General theorem on Conjugate nets

One of the important applications of the pseudo-normal congruence is found in the projective generalization of the concept of geodesics in metric differential geometry. In preparation for the study of this generalization, we need certain theorems concerning conjugate nets which we shall establish in the present section.

Any conjugate net on the surface $S$, which we suppose as usual to be referred to its asymptotic net, may be defined by a differential equation of the form

$$
\lambda^{2} d u^{2}-d v^{2}=0,
$$

where $\lambda$ is a function of $(u, v)$ which is nowhere zero in the region under consideration. At each point $y$ of the surface, in fact, the differential equation defines a pair of conjugate directions, which are constructed by joining the point $y$ to the points $y_{u}-\lambda y_{v}$ and $y_{u}+\lambda y_{v}$. If we denote by $\eta_{-1}$ a point of the first, and by $\eta_{1}$ a point of the second of these conjugate tangents, then the expressions for $\eta_{-1}$ and $\eta_{1}$ must be of the form

$$
\begin{aligned}
& \eta_{-1}=y_{u}-\lambda y_{v}+\omega y, \\
& \eta_{1}=y_{u}+\lambda y_{v}+\omega^{\prime} y .
\end{aligned}
$$

We wish now to determine $\omega$ and $\omega^{\prime}$ as functions of $(u, v)$ so that $\eta_{-1}$ and $\eta_{1}$ shall be the minus first and first Laplace transforms respectively of $y$, in the following sense-which is the usual one of the general theory of congruences. The conjugate net is made up of two one-parameter families, corresponding to the two factors of equation (107):

$$
\lambda d u+d v=0, \quad \lambda d u-d v=0 .
$$

The tangents to the curves of the first family are the lines $y \eta_{-1}$, those of the second family are the lines $y \eta_{1}$. As the point $y$ traces a curve of the second family, the line $y \eta_{-1}$ generates a developable surface, and the points of the edge of regression of this developable are the minus first Laplace transforms of the corresponding points $y$. Similarly, the first Laplace transform of the point $y$ is the point in which the line $y \eta_{1}$ touches the developable generated by the tangents to the curves of the second of the families (109), constructed at the points of a fixed curve of the first family. We wish to determine the functions $\omega$ and $\omega^{\prime}$ so that equations (108) shall represent the minus first and first Laplace transforms of the point $y$.

As the point $y$ traces a curve of the second family, the line $y \eta_{-1}$ generates a developable, and the point $\eta_{-1}$ describes a curve on this developable. A point on the tangent to this curve is obviously given by the expression

$$
Y=\left(\frac{\partial}{\partial u}+\lambda \frac{\partial}{\partial v}\right)\left(y_{u}-\lambda y_{v}+\omega y\right) \text {. }
$$


The differential operator used here corresponds to differentiation along the curves of the second of families (109). Performing the differentiations indicated, and using equations (4) to replace $y_{u u}$ and $y_{v v}$ by their values in terms of $y_{u}, y_{v}$, and $y$, we find without difficulty that

$$
Y=\left(2 \lambda^{2} a^{\prime}+\omega\right) y_{u}-\left(2 b+\lambda_{u}+\lambda \lambda_{v}-\lambda \omega\right) y_{v}+() y,
$$

where the coefficient of $y$ does not concern us here. We wish the line $y Y$ to coincide with the line $y \eta_{-1}$, so that the coefficients of $y_{u}$ and $y_{v}$ in the expressions for $Y$ and $\eta_{-1}$ must be proportional to each other. This condition yields at once the equation

$$
2 \lambda^{2} a^{\prime}+\omega=\frac{2 b+\lambda_{u}+\lambda \lambda_{v}-\lambda \omega}{\lambda},
$$

from which we obtain the desired value for $\omega$ :

$$
\omega=\frac{1}{2 \lambda}\left(2 b-2 a^{\prime} \lambda^{3}+\lambda_{u}+\lambda \lambda_{v}\right) .
$$

In the same way we may find that

$$
\omega^{\prime}=\frac{1}{2 \lambda}\left(-2 b-2 c^{\prime} \lambda^{3}+\lambda_{u}-\lambda \lambda_{v}\right) ;
$$

in fact, the expression for $\omega^{\prime}$ may evidently be obtained from that for $\omega$ by replacing everywhere $\lambda$ by $-\lambda$.

We may therefore state the result: the minus first and first Laplace transforms of the conjugate net defined by the differential equations (109),

$$
\lambda d u+d v=0, \quad \lambda d u-d v=0,
$$

are given by the expressions

$$
\begin{aligned}
\eta_{-1} & =y_{u}-\lambda y_{v}+\frac{1}{2 \lambda}\left(2 b-2 a^{\prime} \lambda^{3}+\lambda_{u}+\lambda \lambda_{v}\right) y, \\
\eta_{1} & =y_{u}+\lambda y_{v}+\frac{1}{2 \lambda}\left(-2 b-2 a^{\prime} \lambda^{3}+\lambda_{u}-\lambda \lambda_{v}\right) y .
\end{aligned}
$$

Wilczynski* has called the line joining the minus first and first Laplace transforms of the point $y$ the ray of the point $y$, and the congruence formed by all the rays the ray congruence of the conjugate net. For our present purposes, the ray itself is more important than the actual Laplace transforms $\eta_{-1}$ and $\eta_{1}$, so that we proceed to find the intersections of the ray with the asymptotic tangents. These intersections are obviously obtained by forming two linear combinations of expressions (113), one of the combinations being independent of $y_{v}$, the other of $y_{u}$. In this way we find that the ray of the

* E. J. Wilczynski, The general theory of congruences, these Trans a ctio n s, vol. 16 (1915), pp. 311-327. Cf. §3. 
point $y$ with respect to the conjugate net (109) cuts the asymptotic tangents of $y$ in the points

$$
\begin{aligned}
& \mathfrak{r}=y_{u}-\frac{1}{2 \lambda}\left(2 a^{\prime} \lambda^{3}-\lambda_{u}\right) y, \\
& \mathfrak{z}=y_{v}-\frac{1}{2 \lambda^{2}}\left(2 b+\lambda \lambda_{v}\right) y .
\end{aligned}
$$

We have thus far been writing the differential equation of the conjugate net in the form (106) to avoid irrationalities. If we put $\mu=\lambda^{2}$, equation (106) becomes

$$
\mu d u^{2}-d v^{2}=0,
$$

and the irrational $\sqrt{\mu}$ appears throughout the subsequent discussion. The final formulas (114), however, come out free from radicals, and we may restate our theorem as follows: the ray of the point $y$ with respect to the conjugate net (115) is the line which joins the points

$$
\mathfrak{r}=y_{u}-\frac{1}{4 \mu}\left(4 a^{\prime} \mu^{2}-\mu_{u}\right) y, \quad \mathfrak{z}=y_{v}-\frac{1}{4 \mu}\left(4 b+\mu_{v}\right) y .
$$

We may now treat the ray congruence as a congruence $\Gamma$, for which

$$
\alpha=\frac{1}{4 \mu}\left(4 b+\mu_{v}\right), \quad \beta=\frac{1}{4 \mu}\left(4 a^{\prime} \mu^{2}-\mu_{u}\right),
$$

by the methods of the present paper. As an illustration, we shall seek the condition that the conjugate net have equal point invariants. We may use Wilczynski's theorem,* that in this case the developables of the ray congruence correspond to a conjugate net on the surface $S$, or, in our terminology, that the ray congruence is harmonic to the surface $S$. Referring to equation (29), which gives the required condition in the form $\alpha_{u}-\beta_{v}=0$, we obtain at once the theorem: the conjugate net defined by equation (115) has equal point invariants if and only if

that is,

$$
\frac{\partial}{\partial u}\left(\frac{4 b}{\mu}+\frac{\mu_{v}}{\mu}\right)=\frac{\partial}{\partial v}\left(4 a^{\prime} \mu-\frac{\mu_{u}}{\mu}\right)
$$

$$
\frac{\partial^{2} \log \mu}{\partial u \partial v}+2 \frac{\partial}{\partial u}\left(\frac{b}{\mu}\right)-2 \frac{\partial}{\partial v}\left(a^{\prime} \mu\right)=0 .
$$

The solution of this differential equation is equivalent to the determination of all conjugate nets with equal point invariants on a given surface.

We have elsewhere $\dagger$ defined a new conjugate net which is uniquely deter-

${ }^{*}$ E. J. Wilczynski, loc. cit., page 319.

† G. M. Green, Projective differential geometry of one-parameter families of space curves, etc., second memoir, American Journal of Mathematics, vol. 38 (1916), p. 314 . 
mined by a given conjugate net, and which we have called the associate conjugate net of the given net. The property that characterizes the associate conjugate net is the following: at each point $y$, the pair of tangents to the two curves of the associate conjugate net which pass through $y$ separate harmonically the pair of tangents to the curves of the given conjugate net at $y$. The relation between the two nets is obviously a reciprocal one: either is the associate of the other. It may be verified without difficulty that the conjugate net associate to the net (115) is defined by the differential equation

$$
\mu d u^{2}+d v^{2}=0 .
$$

Its ray we call the associate ray of the net (115), and it is the line which connects the points

$$
\mathfrak{r}^{\prime}=y_{u}+\frac{1}{4 \mu}\left(4 a^{\prime} \mu^{2}+\mu_{u}\right) y, \quad \mathbb{8}^{\prime}=y_{v}+\frac{1}{4 \mu}\left(4 b-\mu_{v}\right) y .
$$

we have, in the paper just cited,* proved that a conjugate net has equal tangential invariants if and only if its associate conjugate net has equal point invariants; i. e., if and only if its associate ray congruence is harmonic to the surface. Consequently, the conjugate net defined by equation (115) has equal tangential invariants if and only if

$$
\frac{\partial}{\partial u}\left(4 \frac{b}{\mu}-\frac{\mu_{v}}{\mu}\right)=\frac{\partial}{\partial v}\left(4 a^{\prime} \mu+\frac{\mu_{u}}{\mu}\right),
$$

that is:

$$
\frac{\partial^{2} \log \mu}{\partial u \partial v}-2 \frac{\partial}{\partial u}\left(\frac{b}{\mu}\right)+2 \frac{\partial}{\partial v}\left(a^{\prime} \mu\right)=0 .
$$

We are now in position to formulate the analytic problem involved in the determination of all congruences $\Gamma$ which may serve as ray congruences for conjugate nets, and the conjugate nets which have such a congruence $\Gamma$ as their common ray congruence. We may write equations (117) in the form

$$
\mu_{u}=4 a^{\prime} \mu^{2}-4 \beta \mu, \quad \mu_{v}=-4 b+4 \alpha \mu,
$$

where $a^{\prime}, b, \alpha, \beta$ are now supposed to be known functions of $(u, v)$, and $\mu$ is to be determined. We shall find that the functions $\alpha$ and $\beta$ are not arbitrary; in other words, there is a special class of congruences $\Gamma$ associated with any given surface, which can serve as ray congruences for conjugate nets on the surface. A necessary and sufficient condition that equations (124) have a common solution $\mu$ is that the equation

$$
\frac{\partial}{\partial v}\left(a^{\prime} \mu^{2}-\beta \mu\right)=\frac{\partial}{\partial u}(-b+\alpha \mu)
$$

\footnotetext{
* Page 320, especially the first footnote, page 311 .
} 
be satisfied identically by such a solution. On carrying out the differentiations, and collecting terms, we find that this condition of integrability may be written as follows:

$$
\left(a_{v}^{\prime}+4 a^{\prime} \alpha\right) \mu^{2}-\left(8 a^{\prime} b+\alpha_{u}+\beta_{v}\right) \mu+\left(b_{u}+4 b \beta\right)=0 .
$$

Two cases are possible:

1. This equation is an identity in $\mu, u$, and $v$, in which event the three coefficients of the quadratic in $\mu$ are all zero; the system of differential equations is completely integrable.

2. The quadratic may be solved for $\mu$; we include the case in which the coefficient of $\mu^{2}$ vanishes, when the quadratic reduces to a linear equation, and we discard the solution $\mu=0$ which arises when the last term of the quadratic is zero.

In case 1 , let us assume first that the surface is not ruled; we have, since $a^{\prime} b \neq 0$,

$$
\alpha=-\frac{a_{\mathrm{v}}^{\prime}}{4 a^{\prime}}, \quad \beta=-\frac{b_{u}}{4 b}, \quad 8 a^{\prime} b+\alpha_{u}+\beta_{v}=0 .
$$

The values for $\alpha$ and $\beta$ show that the assigned congruence is not arbitrary; it must be, in fact, the canonical congruence of the first kind ( $\$ 12)$. The third equation of condition shows that the surface itself is also restricted; in fact, if $\alpha$ and $\beta$ be replaced by their values, the condition is without difficulty reduced to

$$
\frac{\partial^{2} \log \left(a^{\prime} b\right)}{\partial u \partial v}=32 a^{\prime} b .
$$

If we put $\phi=\log \left(a^{\prime} b\right)$, equation (127) may be written

$$
\frac{\partial^{2} \phi}{\partial u \partial v}=32 e^{\phi} \quad\left(\phi=\log \left(a^{\prime} b\right)\right),
$$

a differential equation which arises frequently both in geometry and in analysis.* The solution of this equation will determine the function $\phi$, and hence a class of surfaces for which equation (127) holds. For each solution $\phi$, the function $\mu$ which determines the conjugate net may now be obtained by a quadrature from equations (124), which in this case form a completely integrable system in virtue of equations (126), since the condition of integrability (125) is satisfied identically in $\mu, u, v$. Since the solution of the com-

* The substitution $u=x+i y, v=x-i y$ converts the equation into

$$
\frac{\partial^{2} \varphi}{\partial x^{2}}+\frac{\partial^{2} \varphi}{\partial y^{2}}=32 e^{\phi}
$$

in which form it is met in a number of investigations, some of them quite recent. For the integration of the equation see L. Lichtenstein, Integration der Differentialgleichung $\Delta_{2} u=k e^{*}$ auf geschlossenen Flächen, A c t a M a th e m a t i c a, vol. 40 (1915), pp. 1-34. 
pletely integrable system in question involves one arbitrary constant, we infer that for each surface of the class defined by equation (127), the canonical congruence of the first kind will serve as a ray congruence for a one-parameter family of conjugate nets on that surface.

We have thus far supposed that the surface $S$ is not ruled; we may dispose very briefly of the case in which it is. Suppose, for instance, that $a^{\prime}=0$. Then the coefficient of $\mu^{2}$ is zero, and the vanishing of the other coefficients is expressed by the two equations

$$
\beta=-\frac{b_{u}}{4 b}, \quad \alpha_{u}+\beta_{v}=0 .
$$

In other words, $\beta$ is fixed, while $\alpha$ and $b$ are connected by the equation

$$
\frac{\partial^{2} \log b}{\partial u \partial v}=4 \alpha_{u}
$$

Therefore, for any ruled surface which is not a quadric, there exists a class of congruences $\Gamma$ for which

$$
\alpha=\frac{b_{v}}{4 b}+A(v), \quad \beta=-\frac{b_{u}}{4 b},
$$

where $A(v)$ is an arbitrary function of $v$, each congruence being the ray congruence for a one-parameter family of conjugate nets on the surface.

For a quadric, $a^{\prime}$ and $b$ both vanish, so that $\alpha_{u}+\beta_{v}=0$ is the condition that system. (124) be completely integrable. Therefore, for a quadric, each of the congruences $\Gamma$ for which $\alpha_{u}+\beta_{v}=0$ is the ray congruence for a oneparameter family of conjugate nets on the quadric.

In case 2 , the surface $S$ may be arbitrary, and even of the special class just considered. The given congruence $\Gamma$, however, is restricted. In fact, any solution of equations (124) must be a non-vanishing root of the quadratic (125) in $\mu$; a root of (125), however, need not satisfy equations (124). In order that such a root may afford a solution of equations (124), $\alpha$ and $\beta$ must satisfy the two partial differential equations of the second order obtained by substituting the value of $\mu$ in both of equations (124). These two partial differential equations in $\alpha, \beta$ are of the form

$$
\begin{aligned}
& \alpha_{u u}+\beta_{u v}=F\left(\alpha, \beta, \alpha_{u}, \beta_{u}, \alpha_{v}, \beta_{v}, u, v\right), \\
& \alpha_{u v}+\beta_{v v}=F^{\prime}\left(\alpha, \beta, \alpha_{u}, \beta_{u}, \alpha_{v}, \beta_{v}, u, v\right),
\end{aligned}
$$

where $F$ and $F^{\prime}$ are explicit functions of the arguments indicated, except that $u, v$ enter through $a^{\prime}, b$ and their derivatives. There are obviously no restrictions required, that these equations may have solutions $\alpha, \beta$. In case 2 , therefore, there exists for any surface $S$ whatever a class of congruences $\Gamma$, 
defined by equations (128), each of which may serve as a ray congruence for some conjugate net on $S$, and in general for only one.*

\section{Pseudo-geodesics, union curves, and generalized surfaces of Voss}

If a geodesic on a surface be defined as a curve, the osculating plane of which at each point contains the normal to the surface at that point, a projective generalization of the definition is not difficult once a substitute has been provided for the normal. We shall call a pseudo-geodesic any curve on the surface, having the property that its osculating plane at each point contains in it the pseudo-normal of the surface at that point.

A generalization of this type has been made by Miss Pauline Sperry, $\dagger$ who replaces the normal congruence by any congruence $\Gamma^{\prime}$ of lines $l^{\prime}$, a line $l^{\prime}$ protruding from each point of the surface $S$. We wish to write Miss Sperry's fundamental equations in the notation of the present paper; since our demonstration thereof is very brief, it may be worth while to give it here in full.

The line $l^{\prime}$ joins the points $y$ and

$$
z=y_{u v}-\alpha y_{u}-\beta y_{v} .
$$

Any curve on the surface may be defined by parametric equations $u=u(t)$, $v=v(t)$. We shall denote the curve by $C_{t}$, putting in evidence the parameter $t$. We wish a necessary and sufficient condition that the osculating plane of $c_{t}$ at $y$ contain in it the line $l^{\prime}$; a curve having this property is called by Miss Sperry a union curve of the congruence $\Gamma^{\prime}$. The osculating plane of $C_{t}$ is determined by the three points $y, d y / d t, d^{2} y / d t^{2}$, where

$$
\frac{d y}{d t}=y_{u} \frac{d u}{d t}+y_{v} \frac{d v}{d t}
$$

* In any event, there cannot be more than two conjugate nets on $S$ having the same ray congruence, since $\mu$ must be a solution of the quadratic (125). The words in general in our italicized statement mean except when certain equations are satisfied, so that if there can be two conjugate nets on $S$ having the same ray congruence, the surface must be of special type. That such surfaces exist we may infer from a theorem proved in our second memoir on conjugate nets, American Journal of Mathematics, vol. 38 (1916), p. 318, to the effect that on a quadric, and only on a quadric, a pair of associated conjugate nets have the same ray congruence. Whether the quadrics are the only surfaces on which two conjugate nets may have the same ray congruence we can not say definitely; the evidence is very strong, however, that there exists a class of surfaces, distinct from the quadrics, for which certain congruences $\Gamma$ will serve as ray congruences for exactly two conjugate nets each. We do not include in these statements, of course, the canonical congruences of the first kind on the surfaces which arise in case 1 .

$\dagger$ P. Sperry, Properties of a certain projectively defined two-parameter family of curves on $a$ general surface, A merican J ournal of Mathematics, vol. 40 (1918), pp. 213224. 


$$
\begin{aligned}
\frac{d^{2} y}{d t^{2}} & =y_{u u}\left(\frac{d u}{d t}\right)^{2}+2 y_{u v} \frac{d u}{d t} \frac{d v}{d t}+y_{v v}\left(\frac{d v}{d t}\right)^{2}+y_{u} \frac{d^{2} u}{d t^{2}}+y_{v} \frac{d^{2} v}{d t^{2}} \\
& =2 y_{u v} \frac{d u}{d t} \frac{d v}{d t}+\left[\frac{d^{2} u}{d t^{2}}-2 a^{\prime}\left(\frac{d v}{d t}\right)^{2}\right] y_{u}+\left[\frac{d^{2} v}{d t^{2}}-2 b\left(\frac{d u}{d t}\right)^{2}\right] y_{v}+\text { ( ) } y,
\end{aligned}
$$

the actual value for the coefficient of $y$ being immaterial. A necessary and sufficient condition, then, that the curve $C_{t}$ be a union curve of the congruence $\Gamma^{\prime}$ is that the points $y, z, d y / d t, d^{2} y / d t^{2}$ lie in a plane, or in other words that in the expressions for $z, d y / d t, d^{2} y / d t^{2}$ the determinant of the coefficients of $y_{u v}, y_{u}, y_{v}$ vanish identically. That is,

$$
\left|\begin{array}{ccc}
1 & -\alpha & -\beta \\
0 & \frac{d u}{d t} & \frac{d v}{d t} \\
2 \frac{d u}{d t} \frac{d v}{d t} & \frac{d^{2} u}{d t^{2}}-2 a^{\prime}\left(\frac{d v}{d t}\right)^{2} & \frac{d^{2} v}{d t^{2}}-2 b\left(\frac{d u}{d t}\right)^{2}
\end{array}\right|=0 .
$$

We may expand this determinant, and state the result in the following form: $A$ curve $C_{t}$ defined by the parametric equations $u=u(t), v=v(t)$ is a union curve of the congruence $\Gamma^{\prime}$ if and only if the functions $u$ and $v$ satisfy the differential equation of the second order

$$
\begin{aligned}
\frac{d u}{d t} \frac{d^{2} v}{d t^{2}}-\frac{d v}{d t} \frac{d^{2} u}{d t^{2}}-2 b\left(\frac{d u}{d t}\right)^{3}+2 \beta\left(\frac{d u}{d t}\right)^{2} \frac{d v}{d t} & -2 \alpha \frac{d u}{d t}\left(\frac{d v}{d t}\right)^{2}+2 a^{\prime}\left(\frac{d v}{d t}\right)^{3}=0
\end{aligned}
$$

If we choose $u$ as the independent variable, writing the equation of $C_{t}$ in the form $v=f(u)$, equation (129a) becomes

$$
\frac{d^{2} v}{d u^{2}}=2 b-2 \beta \frac{d v}{d u}+2 \alpha\left(\frac{d v}{d u}\right)^{2}-2 a^{\prime}\left(\frac{d v}{d u}\right)^{3} .
$$

Equations (129a) and (129b) are essentially those derived by Miss Sperry. They show that there are altogether a two-parameter family of union curves on the surface.

We now proceed to show the desirability of replacing the congruence of normals by a covariant congruence which is not only uniquely determined in a projective way by the surface-as is, for instance, the directrix congruence of the second kind, which Miss Sperry proposes* as a substitute of this nature -but has in addition the property, in common with the congruence of normals, of being conjugate to the surface. The congruence of pseudo-normals which we have introduced above, in $\S 15$, is the simplest congruence of this kind.

${ }^{*}$ Loc. cit., page 216. 
Whether we employ this congruence or any other which is conjugate to the surface, however, is merely a matter of convenience.

A surface of Voss is a surface having the property, that the two-parameter family of geodesics contains two one-parameter families which together constitute a conjugate net. As is well known, this conjugate net of geodesics on a surface of Voss has equal tangential invariants. It is in demanding the preservation of this property in any projective generalization of surfaces of Voss that we discover the necessity of providing as a substitute for the congruence of normals a congruence which is likewise conjugate to the surface.

Let us suppose, first, that we replace the congruence of normals by any congruence $\Gamma^{\prime}$ of lines $l^{\prime}$, without assuming the congruence to be conjugate to the surface. We shall obtain a necessary and sufficient condition that there exist on the surface a conjugate net composed entirely of union curves of the congruence $\Gamma^{\prime}$. Any conjugate net may be defined by a differential equation of the form (107), or, what is the same thing, by the two equations

$$
\frac{d v}{d u}=-\lambda, \quad \frac{d v}{d u}=\lambda
$$

We wish to find the functions $\lambda$ such that equation (129b) is satisfied when $d v / d u$ is replaced therein by either $-\lambda$ or $+\lambda$. We have from (130)

$$
\frac{d^{2} v}{d u^{2}}=-\lambda_{u}+\lambda \lambda_{v}, \quad \frac{d^{2} v}{d u^{2}}=\lambda_{u}+\lambda \lambda_{v} .
$$

Substituting, then, $d v / d u=-\lambda$ and $d v / d u=\lambda$ in turn in equation (129b), we obtain the two equations

$$
\begin{aligned}
-\lambda_{u}+\lambda \lambda_{v} & =2 b+2 \beta \lambda+2 \alpha \lambda^{2}+2 a^{\prime} \lambda^{3}, \\
\lambda_{u}+\lambda \lambda_{v} & =2 b-2 \beta \lambda+2 \alpha \lambda^{2}-2 a^{\prime} \lambda^{3} .
\end{aligned}
$$

Adding and subtracting these, in the proper order, we obtain the equivalent, and much simpler, pair:

$$
\lambda \lambda_{v}=2 b+2 \alpha \lambda^{2}, \quad \lambda_{u}=-2 \beta \lambda-2 a^{\prime} \lambda^{3} .
$$

If in these equations we replace $\lambda^{2}$ by $\mu$, they become

$$
\mu_{v}=4 b+4 \alpha \mu, \quad \mu_{u}=-4 \beta \mu-4 a^{\prime} \mu^{2} .
$$

Since $\Gamma^{\prime}$ was the given congruence, in terms of which the union curves were defined, $\alpha$ and $\beta$ are known functions, and $\mu$ is to be determined from equations $\left(131^{\prime}\right)$. The analytic problem involved in the determination of $\mu$ was discussed in the preceding section. ${ }^{*}$ Since the functions $\alpha$ and $\beta$ in the present case are fixed, and, in any case which is of interest in the general theory of surfaces, are determined by system (4), $\dagger$ it is only for particular surfaces that

* Equations (131') become equations (124) if the sign of $\mu$ be changed.

$\dagger$ That is, the congruence $\Gamma^{\prime}$ is a covariant congruence. 
a function $\mu$ exists which satisfies equations $\left(131^{\prime}\right)$. The condition, which is complicated, consists of the two equations (128) at the end of the preceding section. Each congruence $\Gamma^{\prime}$, or better, each set of properties defining a congruence $\Gamma^{\prime}$ uniquely for any surface, has therefore associated with it a class of surfaces, on each of which there exists a conjugate net formed of union curves. Thus, there is a class of surfaces, which we shall call generalized surfaces of Voss, on each of which exists a conjugate net composed entirely of pseudo-geodesics. Similarly, there exists another class of surfaces each of which has on it a conjugate net formed of union curves of the directrix congruence of the second kind.

To return to our main object; in deriving equations $\left(131^{\prime}\right)$, we have not assumed that the congruence $\Gamma^{\prime}$ is conjugate to the surface. Solving these equations for $\alpha$ and $\beta$, we find that

$$
\alpha=-\frac{1}{4 \mu}\left(4 b-\mu_{v}\right), \quad \beta=-\frac{1}{4 \mu}\left(4 a^{\prime} \mu^{2}+\mu_{u}\right) .
$$

On referring to equation (121), we may conclude that the line $l$ which is reciprocal to the line $l^{\prime}$ is the associate ray of the conjugate net formed of union curves. We may therefore state the

THEOREM. If a congruence $\Gamma^{\prime}$ has the property that its union curves contain a conjugate net, then this net has for its associate ray congruence the congruence $\Gamma$ which is reciprocal to $\Gamma^{\prime}$. If any one-parameter family of union curves of a congruence $\Gamma^{\prime}$ has for its associate ray congruence* the congruence $\Gamma$ reciprocal to $\Gamma^{\prime}$, then the family conjugate to the said family of union curves is also composed of union curves.

We shall now demand further, in analogy to the property of surfaces of Voss already stated, that the conjugate net formed of union curves have equal tangential invariants. In virtue of the theorem just proved, the congruence $\Gamma$ will then be harmonic to the surface, since the associate ray congruence of a conjugate net is harmonic to a surface if and only if the conjugate net has equal tangential invariants. $\dagger$ The congruence $\Gamma^{\prime}$, which is reciprocal to $\Gamma$, will therefore be conjugate to the surface. Whence the following

THEOREM. If the union curves of a congruence $\Gamma^{\prime}$ contain a conjugate net, then a necessary and sufficient condition that this net have equal tangential invariants is that the congruence $\Gamma^{\prime}$ be conjugate to the surface.

We have therefore shown that in order to be able to generalize a very fundamental property of geodesics, we must supply a substitute for the congruence

\footnotetext{
${ }^{*}$ By the associate ray congruence of a one-parameter family of curves we mean the associate ray congruence of the conjugate net of which the family is a component.

† G. M. Green, Projective differential geometry of one-parameter families of space curves, etc., second memoir, American Journal of Mathematics, vol. 38 (1916), p. 320, top, and especially the first footnote, p. 311.
} 
of normals which is conjugate to the surface. In terms of the congruence of pseudo-normals, which has the desired property, we may finally state the theorem, that on a generalized surface of Voss, the conjugate net of pseudogeodesics has equal tangential invariants.

\section{Dual considerations; curves of Darboux and Segre; axis and ray CONGRUENCES}

We have already seen, in $\S 6$, that when a surface $S$ is transformed into a surface $S_{1}$ by a polar reciprocation, point and tangent plane of $S$ going over into corresponding tangent plane and point of $S_{1}$, a pair of reciprocal congruences $\Gamma$ and $\Gamma^{\prime}$ for $S$ are transformed respectively into a pair of reciprocal congruences for $S_{1}, \Gamma$ becoming a congruence $\Gamma_{1}^{\prime}$, and $\Gamma^{\prime}$ becoming a congruence $\Gamma_{1}$. As usual, we say that a curve $C$ on $S$ corresponds to a curve $C_{1}$ on $S_{1}$ if the tangent planes of $S_{1}$ along $C_{1}$ are the planes which correspond to the points of $C$. Then the tangents to $C$ are transformed into the tangents of $S_{1}$ conjugate to the tangents to $C_{1}$. The developable formed by these conjugate tangents along $C_{1}$ we shall call the conjugate developable of $C_{1}$, and its edge of regression the first Laplace transform of $C_{1}$. In fact, we shall say that a point of this edge of regression is the first Laplace transform of the corresponding point of $C_{1}$. These definitions are in conformity with the familiar ones for a conjugate net, or what is the same thing, for either of the one-parameter families which form the net.

It is obvious geometrically that by polar reciprocation of the surface $S$ the osculating plane at a point $y$ of a curve $C$ on $S$ is transformed into the first Laplace transform of the corresponding point $y_{1}$ of the corresponding curve $C_{1}$ of $S_{1}$. Since, moreover, the transform of the congruence $\Gamma^{\prime}$ is the congruence $\Gamma_{1}$, we may formulate a definition dual to that of the union curves: a curve $C_{t}$ is called an adjoint union curve of a congruence $\Gamma$ if the first Laplace transform of each point $y$ of the curve lies on that line $l$ of the congruence $\Gamma$ which corresponds to $y$-that is, the line $l$ which lies in the tangent plane to $S$ at $y$.*

There obviously exist on the surface $S$ a two-parameter family of adjoint union curves for a given congruence $\Gamma$. The differential equation of these curves is easily found as follows. The curve $C_{t}$ may be defined by means of the differential equation

$$
\lambda d u-d v=0,
$$

where $\lambda$ is a function of $(u, v)$; the curve is therefore a member of the oneparameter family of integral curves of this differential equation, and we may as well determine $\lambda$ so that all of these curves shall be adjoint union curves.

* P. Sperry, loc. cit., p. 222, gives this definition of dual union curves. She calls the first Laplace transform of a point of a curve the osculating point. 
The first Laplace transform of the curve $C_{t}$, or the one-parameter family of curves, is defined by the first of equations (113):

$$
\eta_{-1}=y_{u}-\lambda y_{v}+\frac{1}{2 \lambda}\left(2 b-2 a^{\prime} \lambda^{3}+\lambda_{u}+\lambda \lambda_{v}\right) y,
$$

where $\lambda=d v / d u$. We wish this point to lie on the line $l$ which connects the points

$$
\rho=y_{u}-\beta y, \quad \sigma=y_{v}-\alpha y .
$$

A necessary and sufficient condition for this is the vanishing of the determinant formed from the coefficients of $y_{u}, y_{v}$, and $y$ in the expressions for $\eta_{-1}, \rho, \sigma$. On expanding this determinant, we obtain the equation

$$
\lambda_{u}+\lambda \lambda_{v}=-2 b-2 \beta \lambda+2 \alpha \lambda^{2}+2 a^{\prime} \lambda^{3} .
$$

Since $\lambda_{u}+\lambda \lambda_{v}=d \lambda / d u$, we find finally that the adjoint union curves are defined by the differential equation

$$
\frac{d^{2} v}{d u^{2}}=-2 b-2 \beta \frac{d v}{d u}+2 \alpha\left(\frac{d v}{d u}\right)^{2}+2 a^{\prime}\left(\frac{d v}{d u}\right)^{3} .
$$

This equation differs from that of the union curves in having the signs of the coefficients $b$ and $a^{\prime}$ changed, so that the two equations can coincide only when $b$ and $a^{\prime}$ are both zero. We may, in fact, state the theorem, that the union curves of a congruence $\Gamma^{\prime}$ coincide with the adjoint union curves of the reciprocal congruence $\Gamma$ if and only if the surface is a quadric.*

But it may happen that a particular union curve coincides with a corresponding adjoint union curve. In this case, equations (129b) and (132) must have a common particular solution, and this solution must therefore satisfy the two equations

$$
\frac{d^{2} v}{d u^{2}}+2 \beta \frac{d v}{d u}-2 \alpha\left(\frac{d v}{d u}\right)^{2}=0, \quad a^{\prime}\left(\frac{d v}{d u}\right)^{3}-b=0 .
$$

The second of these equations shows that there are three possible values for $\lambda=d v / d u$, giving three one-parameter families of curves which are at the same time union curves and adjoint union curves; the related pairs of reciprocal congruences $\Gamma$ and $\Gamma^{\prime}$ are not arbitrary, however, but partially determined by the requirement that the value of $d v / d u$ obtained from the second of equations (133) satisfy also the first of those equations. This requirement we may express in geometric language as follows. The first of equations (133) may be written

$$
\lambda_{u}+\lambda \lambda_{v}+2 \beta \lambda-2 \alpha \lambda^{2}=0,
$$

* Miss Sperry derives the differential equation of the adjoint union curves directly by the principle of duality, using the system of differential equations adjoint to system (4). The fact that the quadrics are the only identical self-dual surfaces suggests at once our last theorem, from this point of view. 
so that we may substitute for $\lambda_{u}+\lambda \lambda_{v}$ its value, $2 \alpha \lambda^{2}-2 \beta \lambda$, in the expression for $\eta_{-1}$ given by the first of equations (113):

$$
\begin{aligned}
\eta_{-1} & =y_{u}-\lambda y_{v}+\frac{1}{2 \lambda}\left(2 \alpha \lambda^{2}-2 \beta \lambda\right) y \\
& =\rho-\lambda \sigma .
\end{aligned}
$$

We have, of course, used the relation $a^{\prime} \lambda^{3}-b=0$. The equation which we have just obtained merely expresses the fact that the point $\eta_{-1}$ lies on the line $\rho \sigma$, and it is easily seen that this is the only geometric restriction on the line $\rho \sigma$ which is expressed by the first of equations (133)-in the presence, of course, of the second of those equations. But this restriction is already involved in the requirement that the curves be adjoint union curves of the congruence $\Gamma$. We may therefore state the following theorem:

On any non-ruled surface $S$ there exist three one-parameter families of curves defined by the differential equation

$$
b d u^{3}-a^{\prime} d v^{3}=0
$$

each of the families is characterized by the property that the curves of which it consists are at the same time union curves of some congruence $\Gamma^{\prime}$ and adjoint union curves of the reciprocal congruence $\Gamma$.

Having constructed a one-parameter family of curves of the kind just characterized, we may easily construct all the congruences $\Gamma$ of which the curves are adjoint union curves. For, the only requirement on $\Gamma$ is that each line $l$ thereof contain the first Laplace transform of the corresponding point of the surface, with respect to the curve of the family which passes through that point.

We shall call the curves defined by the differential equation (134) the curves of Segre, because the tangents to the three of these curves at a point of the surface were first defined by Segre, ${ }^{*}$ by a method quite different from ours. Another important set of three one-parameter families of curves is that defined by the differential equation

$$
b d u^{3}+a^{\prime} d v^{3}=0
$$

we shall call them the curves of Darboux. They constitute the three families conjugate to the three families of curves of Segre; however, they were discovered by Darboux, $\dagger$ and defined by him in terms of a characteristic property

* C. Segre, Complementi alla teoria delle tangenti coniugate di una superficie, $\mathrm{R}$ e $\mathrm{n} \mathrm{d}$ i c o $\mathrm{n}$ t $\mathrm{i}$ della R. A c cademia dei Lincei, ser. 5, vol. 172 (1908), pp. 405-412. Cf. in particular pp. 409-411.

† G. Darboux, Sur le contact des courbes et des surfaces, B ulletin des S c i e n ce s M a t h e m a ti ques, ser. 2, vol. 4 (1880), pp. 348-384, in particular pp. 356 et seq. 
of their tangents, long before Segre came upon them again, from an entirely different point of view, in the paper already cited.*

Darboux's characterization may be described as follows. There exists a three-parameter family of quadrics having contact of the second order with the surface at a point $y$, and each quadric intersects the surface in a curve having a triple point at $y$. The three tangents at the triple point lie in the tangent plane of the surface, and correspond, not to a single quadric, but to a one-parameter family of quadrics. The three tangents at a triple point may coincide, but, if they do, it must be in one of three directions, which Darboux calls tangents of quadric osculation, and which are defined by the differential equation (135). He calls the integral curves of this differential equation curves of quadric osculation. We may state an equivalent characterization of these curves, in purely geometric terms and independently of the notion of order of contact, as follows: the curves of Darboux are the three one-parameter families of curves defined by the differential equation (135), and are completely characterized by the property that they are the families conjugate to the three families, defined by equation (134), each of which families is composed at the same time of union curves for a congruence $\Gamma^{\prime}$ and adjoint union curves for the reciprocal congruence $\Gamma$.

The curves of Segre are obviously self-dual, as are also the curves of Darboux; that is, if surfaces $S$ and $S_{1}$ correspond to each other by a polar reciprocation, then the curves of Segre on $S$ correspond to the curves of Segre on $S_{1}$. However, the tangents to the curves of Segre, or, as we may call them, the tangents of Segre, do not correspond to the tangents of Segre on the other surface, but to the tangents of Darboux. This follows from the fact that the tangents of a curve on one surface go over into the tangents conjugate to the tangents of the corresponding curve on the other surface. The tangents of Segre are therefore dual to the tangents of Darboux, as was pointed out by Segre in his paper; in fact, he used dual processes to define both kinds of tangents.

The principle of duality will allow us to state for adjoint union curves theorems analogous to those of the preceding section. We need only dualize the concept of ray there described; this leads us to the definition of the axis of a point $y$ of the surface with respect to a conjugate net, as the line of intersection of the osculating planes of the curves of the net at the said point $y$. The axis congruence is the congruence formed of all the axes of the conjugate

* Fubini has recently pointed out how fundamental a part is played by the curves of Darboux - which he calls the curves of Darboux-Segre - in certain problems of projective differential geometry. Cf. his two papers, Invarianti proiettivo-differenziale delle curve tracciate su una superficie e definizione proiettivo-differenziale di una superficie, A $\mathrm{n} n$ ali $\mathrm{d}$ i $\mathrm{M}$ a t em a ti c a , ser. 3, vol. 25 (1916), pp. 229-252, and Applicabilità proiettiva di due superficie, Palermo Rendiconti, vol. 41 (1916), pp. 135-162.

Trans. Am. Math. Soc. 10 
net, and the associate axis congruence of a conjugate net is the axis congruence of the associate conjugate net. It is now easily seen that the first of the two fundamental theorems of the preceding section may be stated thus: if there exists a conjugate net formed of union curves of a congruence $\Gamma^{\prime}$, then the adjoint union curves of the reciprocal congruence $\Gamma$ will likewise contain a conjugate net, the two conjugate nets being associate to each other. Or, in terms of the conjugate nets themselves, the axis congruence of a conjugate net is reciprocal to the associate ray congruence, and the ray congruence is reciprocal to the associate axis congruence. This theorem throws light on a number of particular results previously obtained by the writer for conjugate nets;* one of these is the fact that the axis congruence is conjugate to the surface if and only if the associate ray congruence is harmonic to the surface. $\dagger$ This is now an immediate consequence of the general properties of reciprocal congruences, following as it does from the theorem stated immediately before, and the property proved in $\S 8$, p. 100 , that a congruence $\Gamma$ is harmonic to a surface if and only if its reciprocal congruence $\Gamma^{\prime}$ is conjugate to the surface.

The second fundamental theorem of the preceding section yields by polar reciprocation the following:

If the adjoint union curves of a congruence $\Gamma$ contain a conjugate net, then a necessary and sufficient condition that this net have equal point invariants is that the congruence $\Gamma$ be harmonic to the surface.

No new class of surfaces may be defined by means of this theorem, however, since it is without difficulty seen to be a consequence of the first theorem above and of its own dual, and is consequently equivalent to its dual. It therefore leads to the same surfaces, analogous to the surfaces of Voss, as were defined in the preceding section.

\section{Projective generalization of metric theorems}

In the preceding pages we have several times encountered theorems which have well-known metric theorems as very special cases, and we have repeatedly spoken of projective generalizations of metric theorems, especially in connection with the congruence of pseudo-normals. There are, of course, several ways in which metric theorems may be generalized. First, there is the obvious method of projecting the configuration, so that the plane at infinity-if we are considering the configuration in space of three dimensions-becomes any plane of space; or, what is the same thing, to select any plane of the projective space as a "plane at infinity." Then there is a method which may be re-

* G. M. Green, Projective differential geometry of one-parameter families of space curves, etc., second memoir. American Journal of Mathematics, vol. 38 (1916), pp. 287-324.

† Loc. cit., p. 320. 
garded as a further generalization in this direction, viz., that in which a quadric is chosen as an "absolute," and distance and angle measured with reference to this quadric. Interesting results may, of course, be obtained in this way; in the present paper, however, we have had in mind no such direct generalizations, but have sought rather to obtain results the statement of which resembles strongly that of the corresponding metric theorems. In many cases, a generalization of this kind will serve to throw new light on a metric theorem, especially when the metric theorem, though purporting to deal with an essentially metric situation, is really independent of the metric aspects of the configuration. We have seen a good instance of this remark in $\$ 17$, in the case of surfaces of Voss and the projective generalization of these surfaces.* The present section will be devoted to the suggestion of further similar generalizations, and the formulation of problems in connection therewith. No attempt will be made to provide a substitute for the concept of angle; we shall merely use the pseudo-normal in place of the normal, and describe configurations in terms of this new line. This is the nearest approach we can expect to make to the notion of orthogonality without bringing in an extraneous element such as an absolute quadric.

Once having introduced, in the pseudo-normal congruence, a covariant congruence $\Gamma^{\prime}$ whose developables cut the surface in a conjugate net-we have, at the end of $\S 15$, called the curves of this net the pseudo-normal curves -we are in position to define a number of classes of surfaces. First, we recall that an isothermic surface, i. e., one whose lines of curvature form an isothermal net, is characterized by the fact that the conjugate net formed by its lines of curvature has equal point invariants. We may therefore define a class of surfaces, which we shall call pseudo-isothermic, by the property that the conjugate net formed of its pseudo-normal curves have equal point invariants. It is not difficult to write out the analytic condition that a surface referred to its asymptotics be pseudo-isothermic. With reference to the results of $\S 8$, we observe from equations (98) and (36) that the differential equation of the pseudo-normal curves may be written

$$
\left[\theta_{u u}-2 b \theta_{v}+\left(f-2 b_{v}\right) \theta\right] d u^{2}-\left[\theta_{v v}-2 a^{\prime} \theta_{u}+\left(g-2 a_{u}^{\prime}\right) \theta\right] d v^{2}=0,
$$

* Perhaps a better example would be afforded by the well-known proposition, that a geodesic is a line of curvature if and only if it is a plane curve. This is only a particular case of the projective theorem, due to Miss Sperry, that a union curve of a congruence $\Gamma^{\prime}$ is a $\Gamma^{\prime}$-curve if and only if it is a plane curve. To prove this theorem, we observe that if a union curve is plane, the corresponding ruled surface of the congruence $\Gamma^{\prime}$ is developable-is, in fact, the plane of the curve -so that the curve is a $\Gamma^{\prime}$-curve. Also, if a curve is a union curve of $\Gamma^{\prime}$, its osculating planes must be tangent to the corresponding ruled surface of $\Gamma^{\prime}$, which ruled surface is developable if the curve is a $\Gamma^{\prime}$-curve; i. e., the osculating planes of the $\Gamma^{\prime}$-curve envelop the developable, yet that curve is not the edge of regression of the developable, which therefore has no edge of regression and is consequently a plane.

Of course, a plane $\Gamma^{\prime}$-curve is not necessarily a union curve of the cungruence $\Gamma^{\prime}$. 
where

$$
\theta=\frac{1}{\sqrt{a^{\prime} b}}
$$

so that, from equation (119), we infer that a surface $S$, referred to its asymptotic curves, is pseudo-isothermic if and only if

$$
\frac{\partial^{2} \log \mu}{\partial u \partial v}+2 \frac{\partial}{\partial u}\left(\frac{b}{\mu}\right)-2 \frac{\partial}{\partial v}\left(a^{\prime} \mu\right)=0,
$$

where

$$
\mu=\frac{\theta_{u u}-2 b \theta_{v}+\left(f-2 b_{v}\right) \theta}{\theta_{v v}-2 a^{\prime} \theta_{u}+\left(g-2 a_{u}^{\prime}\right) \theta}, \quad \theta=\frac{1}{\sqrt{a^{\prime} b}} .
$$

It is possible, therefore, to define a projective analogue for isothermality, but only for the net of pseudo-normal curves. In another paper, ${ }^{*}$ we have shown that, in the most general case, the property of isothermality for a net of curves on a non-developable surface may be broken up into two parts, first, the property of orthogonality, which is metric, and second, a purely projective property, embodied in the general relation $R . \dagger$ In order, then, to formulate a projective generalization of isothermal nets, it would be necessary to provide a substitute for the property of orthogonality. This may be done by replacing the net of minimal curves on the surface by another covariant net, defined projectively; there are a large number of possibilities, and we shall not attempt to suggest any particular one of them here. It would be desirable, however, to make the definition compatible with that of pseudo-isothermic surfaces just formulated.

Another metric configuration which will lend itself to projective generalization is that of triply orthogonal systems of surfaces. By means of their characteristic property, as involved in Dupin's theorem, we may describe these systems as consisting of three one-parameter families of surfaces which cut each other in their lines of curvature. We may therefore define what we shall call a pseudo-orthogonal triple system of surfaces as consisting of three one-parameter families of surfaces, each surface being cut by the members of the two other families in its pseudo-normal curves. A starting point for the study of such systems will be found in the author's dissertation. $f$ However, in order to make the discussion feasible from this point of view, it would be necessary to obtain the condition that the parametric curves on a surface be the pseudo-normal curves. $\S$ Of course, we can not expect to be able to

* G. M. Green, Some geometric characterizations of isothermal nets on a curved surface, these Trans a c t i on s, vol. 18 (1917), pp. 480-488.

$\dagger \mathrm{Cf}$. the paper just cited, or the references given on Page 87 of the present memoir.

¥ G. M. Green, Projective differential geometry of triple systems of surfaces. Columbia dissertation, private edition, Lancaster, Pa., 1913, pp. 1-27.

$\S$ See above, end of $\S 15$. 
carry the analogy very far, except in certain directions, because a large part of the theory of triply orthogonal systems is based on elementary theorems on differential equations which are intimately bound up with the notion of orthogonal trajectories. For this reason, for instance, there is no differential equation of the third order analogous to the one which defines the families of Lamé; and it is also very unlikely that Darboux's theorem, to the effect that two one-parameter families which intersect each other orthogonally in lines of curvature determine a triply orthogonal system, is susceptible of a projective generalization in connection with pseudo-orthogonal triple systems. However, with reference to this configuration, it is highly desirable to obtain a condition that a rectilinear congruence be a pseudo-normal congruence, and if it is, to determine all the surfaces of which it is the pseudo-normal congruence. These investigations are all likely to be of very great difficulty not only because of analytic, but also because of geometric complications. As a particular type of pseudo-orthogonal triple systems we may mention those formed of pseudo-isothermic surfaces, which will yield a generalization of isothermic triple systems.

Since these generalizations would probably lead to very great complications, it may be worth while to suggest another, which is undoubtedly of sufficient simplicity, and has in fact already received some attention from geometers. We refer to general conjugate triple systems, i. e., triple systems in which each surface is cut in a conjugate net by the surfaces of the other two families. As a generalization of isothermic triple systems which we believe has not yet been made, we suggest conjugate triple systems in which the conjugate nets of intersection have equal point invariants. The study of such a system would be based on the consideration of a completely integrable system of partial differential equations of the form

where

$$
\begin{aligned}
y_{u u} & =a^{(11)} y_{u}+b^{(11)} y_{v}+c^{(11)} y_{w}+d^{(11)} y, \\
y_{v v} & =a^{(22)} y_{u}+b^{(22)} y_{v}+c^{(22)} y_{w}+d^{(22)} y, \\
y_{w w} & =a^{(33)} y_{u}+b^{(33)} y_{v}+c^{(33)} y_{w}+d^{(33)} y, \\
y_{v w} & =\quad b^{(23)} y_{v}+c^{(23)} y_{w}+d^{(23)} y, \\
y_{w u} & =a^{(31)} y_{u} \\
y_{u v} & =a^{(12)} y_{u}+b^{(12)} y_{v} \quad+c^{(31)} y_{w}+d^{(31)} y,
\end{aligned}
$$

$$
b_{w}^{(23)}=c_{v}^{(23)}, \quad c_{u}^{(31)}=a_{w}^{(31)}, \quad a_{v}^{(12)}=b_{u}^{(12)},
$$

and where the coefficients are further restricted by the conditions of complete integrability.*

* Cf. G. M. Green, Columbia dissertation, pp. 3 et seq. 
Besides the generalized surfaces of Voss and the pseudo-isothermic surfaces already defined, we shall mention a few other possible generalizations which may lead to interesting investigations. A surface of Weingarten is one whose normals form a $W$-congruence; a generalized surface of Weingarten, then, may be defined, as one whose pseudo-normals form a $W$-congruence. The question arises at once, as to whether these surfaces have a property similar to that of the $W$-surfaces themselves, viz., that a functional relation exists between their principal radii of curvature.

Another important class of surfaces encountered in metric differential geometry are the surfaces of Guichard, which are the focal sheets of congruences of Guichard, i. e., congruences whose developables correspond to the lines of curvature on both focal sheets. A generalized congruence of Guichard, then, is a congruence whose developables correspond to the pseudo-normal curves of its focal sheets; these focal sheets may be called generalized surfaces of Guichard. The connection between surfaces of Guichard and surfaces of Voss may possibly have an analogue in a similar relation between the generalized surfaces of Guichard and Voss.

One other point is undoubtedly worth mentioning, viz., that the transformation theory of the surfaces which we have defined, and of others which will readily suggest themselves, will repay investigation. The analytic apparatus of the present memoir, moreover, affords a new method of approach to questions of this nature.

\section{Fundamental equations of the theory of surfaces}

The metric differential geometry of surfaces may be regarded as founded on the Gauss differential equations, under which designation we include not only the three equations to which that name is ordinarily applied, but two more which express the first derivatives of the direction cosines of the normal in terms of the first derivatives of the coördinates of a point of the surface. The first three of the equations referred to give the second derivatives of the coördinates in terms of the first derivatives and the direction cosines of the normal. With any other line chosen in place of the normal, it is possible to set up a system of differential equations analogous to the Gauss equations, which may serve as a basis for the projective differential geometry of surfaces.

Let the surface be defined in homogeneous coördinates by the equations

$$
y^{(k)}=y^{(k)}(u, v) \quad(k=1,2,3,4),
$$

the parametric net being any net whatever, and not necessarily asymptotic as heretofore. Then the points $y, y_{u}, y_{v}$ determine the tangent plane to the surface at $y$, and if $z^{(k)}=z^{(k)}(u, v)$ be selected as any point outside the tangent plane any point in space will be linearly dependent on the four points $y, y_{u}$, 
$y_{v}, z$. Therefore the quantities $y_{u u}, y_{u v}, y_{v v}, z_{u}, z_{v}$ will each be expressible linearly in terms of $y, y_{u}, y_{v}, z$, and we may in fact write the equations

$$
\begin{aligned}
& y_{u u}=a^{(11)} y_{u}+b^{(11)} y_{v}+c^{(11)} y+d^{(11)} z, \\
& y_{u v}=a^{(12)} y_{u}+b^{(12)} y_{v}+c^{(12)} y+d^{12)} z, \\
& y_{v v}=a^{(22)} y_{u}+b^{(22)} y_{v}+c^{(22)} y+d^{(22)} z, \\
& z_{u}=m y_{u}+n y_{v}+p y+q z, \\
& z_{v}=m^{\prime} y_{u}+n^{\prime} y_{v}+p^{\prime} y+q^{\prime} z .
\end{aligned}
$$

The coefficients of the right-hand member of any one of these equations are functions of $(u, v)$ which may be obtained by substitution of the four values for $y$ and $z$ in that equation, which yields four linear equations in the coefficients, solvable for those coefficients because the determinant

$$
W=\left|y_{u}, y_{v}, y, z\right|,
$$

formed by the quantities $y_{u}^{(k)}, y_{v}^{(k)}, y^{(k)}, z^{(k)}(k=1,2,3,4)$, is different from zero.

Equations (139) are the analogue of the Gauss differential equations, the line $y z$ taking the place of the normal. We have not chosen the point $z$ in any specific way with reference to the surface; the choice may well be decided by the requirements of the particular problem to which the equations are applied.

If the parametric curves are asymptotic, the first three of equations (139) may be written

$$
y_{u u}=-2 b y_{v}-f y, \quad y_{u v}=\alpha y_{u}+\beta y_{v}+z, \quad y_{v v}=-2 a^{\prime} y_{u}-g y,
$$

which are merely equations (4) and ( 7 ) taken together.

We know that, given the surface $S$, equations (139) exist; we now proceed to the converse question, as to the determination of a surface by means of a system of differential equations of the form (139) assigned at random. Obviously, certain conditions of complete integrability must be satisfied by the coefficients of these equations. We note first of all that the system has a certain characteristic form, viz., that the quantities $y_{u}, y_{v}, y, z$, which we have elsewhere called primary derivatives, ${ }^{*}$ appear in the right-hand members, while the left-hand members consist of all the first order derivatives of these primary derivatives. Consequently, by the general theory, this system will be completely integrable, with a set of four linearly independent solutions $y^{(k)}$ and four linearly independent solutions $z^{(k)}$, provided only that the ambiguous

* G. M. Green, The linear dependence of functions of several variables, and completely integrable systems of homogeneous linear partial differential equations, these T r a n a c t i o n s, vol. 17.(1916), pp. 483-516. Cf. 499. 
expressions for the second derivatives of the primary derivatives be identical.* For instance, the derivative $z_{u v}$ may be calculated in two ways from the last two of equations (139), and each of the two expressions for $z_{u v}$ is expressible linearly in terms of $y_{u}, y_{v}, y, z$ in virtue of equations (139). On equating these two expressions, we obtain an equation of the form

$$
\alpha y_{u}+\beta y_{v}+\gamma y+\delta z=0
$$

and since we wish the determinant $W=\left|y_{u}, y_{v}, y, z\right|$ to be different from zero, we must have $\alpha=\beta=\gamma=\delta=0$. This yields the last four of the following conditions; the first four and the second four respectively arise from a similar procedure with the derivatives $y_{u u v}$ and $y_{u v v}$, each of which may be calculated in two ways:

$$
\begin{gathered}
a_{u}^{(12)}-a_{v}^{(11)}+a^{(12)} b^{(12)}-b^{(11)} a^{(22)}+c^{(12)}=d^{(11)} m^{\prime}-d^{(12)} m, \\
b_{u}^{(12)}-b_{v}^{(11)}+a^{(12)} b^{(11)}+\left(b^{(12)}-a^{(11)}\right) b^{(12)}-b^{(11)} b^{(22)}-c^{(11)} n^{\prime}-d^{(12)} n, \\
c_{u}^{(12)}-c_{v}^{(11)}+a^{(12)} c^{(11)}+\left(b^{(12)}-a^{(11)}\right) c^{(12)}-b^{(11)} c^{(22)}=d^{(11)} p^{\prime}-d^{(12)} p, \\
d_{u}^{(12)}-d_{v}^{(11)}+a^{(12)} d^{(11)}+\left(b^{(12)}-a^{(11)}\right) d^{(12)}-b^{(11)} d^{(22)} \\
=d^{(11)} q^{\prime}-d^{(12)} q, \\
a_{u}^{(22)}-a_{v}^{(12)}+a^{(22)} a^{(11)}+\left(b^{(22)}-a^{(12)}\right) a^{(12)}-b^{(12)} a^{(22)}+c^{(22)} \\
b_{u}^{(22)}-d_{v}^{(12)} m^{\prime}-d^{(22)} m, \\
c_{u}^{(22)}-c_{v}^{(12)}+a^{(22)} b^{(11)}+\left(b^{(22)}-a^{(12)}\right) c^{(12)}-b^{(12)} c^{(22)}
\end{gathered}
$$

$$
=\bar{d}^{(12)} p^{\prime}-d^{(22)} p,
$$$$
d^{(22)}-d_{v}^{(12)}+a^{(22)} d^{(11)}+\left(b^{(22)}-a^{(12)}\right) d^{(12)}-b^{(12)} d^{(22)}
$$$$
=d^{(12)} q^{\prime}-d^{(22)} q,
$$$$
m_{u}^{\prime}-m_{v}+m^{\prime} a^{(11)}+\left(n^{\prime}-m\right) a^{(12)}-n a^{(22)}+p^{\prime}=q m^{\prime}-q^{\prime} m,
$$$$
n_{u}^{\prime}-n_{v}+m^{\prime} b^{(11)}+\left(n^{\prime}-m\right) b^{(12)}-n b^{(22)}-p=q n^{\prime}-q^{\prime} n,
$$

$$
\begin{aligned}
& p_{u}^{\prime}-p_{v}+m^{\prime} c^{(11)}+\left(n^{\prime}-m\right) c^{(12)}-n c^{(22)}=q p^{\prime}-q^{\prime} p, \\
& q_{u}^{\prime}-q_{v}+m^{\prime} d^{(11)}+\left(n^{\prime}-m\right) d^{(12)}-n d^{(22)}=0 .
\end{aligned}
$$

$\dagger$ Idem, ibid. Although the theorems of the paper referred to concern only systems with one dependent variable, they may be extended to apply to system (139), which contains two dependent variables. 
These twelve equations take the place of the Gauss and Mainardi-Codazzi equations of the metric theory. In fact, if the coördinates be made non-homogeneous, and the $z$ 's be replaced by the direction cosines of the normal, the differential equations (139) become the Gauss differential equations, and the twelve conditions of complete integrability (141) reduce to the Gauss-MainardiCodazzi equations.

According to the general theory of completely integrable systems, then, we may state what may be regarded as the fundamental theorem of the projective theory of surfaces: the system of differential equations (139), in which the coefficients satisfy equations (141), determines one and only one surface $S$ except for a projective transformation.

The form of the integrability conditions is interesting. The first eight of them will serve to determine the coefficients of the last two of equations (139) in terms of the coefficients of the first three of equations (139), provided only that $d^{(11)} d^{(22)}-d^{(12)} d^{(12)}$ be different from zero. Now, it is not difficult to see that the differential equation of the asymptotics on $S$ is

$$
d^{(11)} d u^{2}+2 d^{(12)} d u d v+d^{(22)} d v^{2}=0,
$$

so that the condition $d^{(11)} d^{(22)}-d^{(12)} d^{(12)}=0$ corresponds to the case in which the surface is developable. Having, then, determined the coefficients $m, n, p, q, m^{\prime}, n^{\prime}, p^{\prime}, q^{\prime}$ of the last two of equations (139) in terms of the coefficients of the first three of those equations, we may substitute the values found in the last four of equations (141). The twelve integrability conditions may therefore be reduced to four, in which appear only the coefficients of the first three of equations (139). This is quite in accordance with the fact that the integrability conditions for system (4) consist also of four equations, as might have been expected, since through the elimination of $z$ from the first and third of equations (139) by means of the substitution of its value from the second of those equations, the system of five equations is finally reduced to two of the form

$$
\begin{aligned}
& y_{u u}=a y_{u v}+b y_{u}+c y_{v}+d y, \\
& y_{v v}=a^{\prime} y_{u v}+b^{\prime} y_{u}+c^{\prime} y_{v}+d^{\prime} y .
\end{aligned}
$$

The integrability conditions for this system consist of four equations, as may be verified without difficulty. They arise from the equation of the two values obtainable for $y_{u u v v}$.

In the reduction just made, we have of course assumed that $d^{(12)}$ is not zero, i. e., that the parametric net is not conjugate. If the parametric net is conjugate, i. e., if $d^{(12)}=0$, we have in system (139) a system of differential 
equations perfectly symmetric in $u$ and $v$. We need hardly insist on the desirability of preserving symmetry whenever feasible; it is not possible to do this conveniently by means of two equations, as we have pointed out elsewhere.*

By differentiation of the determinant $W=\left|y_{u}, y_{v}, y, z\right|$, it is not difficult to verify that

so that

$$
\begin{aligned}
& W_{u}=\left(a^{(11)}+b^{(12)}+q\right) W, \\
& W_{v}=\left(a^{(12)}+b^{(22)}+q^{\prime}\right) W,
\end{aligned}
$$

$$
\frac{\partial}{\partial v}\left(a^{(11)}+b^{(12)}+q\right)=\frac{\partial}{\partial u}\left(a^{(12)}+b^{(22)}+q^{\prime}\right)
$$

an equation which may also be derived from the integrability conditions (141).

We have thus far left the point $z$ unrestricted; it may, of course, be chosen so that the congruence $\Gamma^{\prime}$ formed by the lines $z y$ have any desired property. We shall point out one choice which may possibly be of some importance, viz., that in which the point $z$ is the same for every point of the surface, i. e., in which the congruence $\Gamma^{\prime}$ consists of the lines passing through a fixed point. In that case, the last two of equations (139) may be reduced to $z_{u}=0, z_{v}=0$, and the integrability conditions (141) consist merely of the first eight equations, with the right-hand members zero.

By using system (139) instead of system (142) we are enabled to make connection between the projective and metric theories of surfaces. We have throughout the present memoir refrained from making any direct application of our results to metric configurations, in which, for instance, the congruence $\Gamma^{\prime}$ may consist of the normals to the surface. A thorough metric study of surfaces and related congruences ought to be undertaken from this point of view; as an indication that such an investigation would not be fruitless, we cite our recent characterization of isothermal nets on a curved surface. $\dagger$

The researches begun in the present memoir are far from complete; in fact, an almost limitless field still remains to be investigated by the methods we have outlined. Unfortunately, there confronts us in many places the unpleasant feature which has always marred the subject of differential geometry, viz., the analytic complications which it seems impossible to dispense with

* G. M. Green, One parameter families of space curves, and conjugate nets on a curved surface, American Journal of Mathematics, vol. 38 (1916), pp. 287-324. Cf. the introduction.

† G. M. Green, Some geometric characterizations of isothermal nets on a curved surface, these Transactions, vol. 18 (1917), pp. 480-488. 
entirely. Nevertheless, we hope that some of the suggestions which we have made in the last two sections may prove to others to be as fruitful as they appear promising to us.

HARVARD UNIVERSITY,

September, 1918. 